\title{
High-Throughput Screen Identifies Host and Microbiota Regulators of Intestinal Barrier Function
}

Inna Grosheva ${ }^{1 *}$, Danping Zheng ${ }^{2,3^{*}}$, Maayan Levy ${ }^{2,4,5}$, Omer Polansky ${ }^{1}$, Alexandra Lichtenstein ${ }^{1}$, Ofra Golani $^{6}$, Mally Dori-Bachash², Claudia Moresi ${ }^{2}$, Hagit Shapiro ${ }^{2}$, Sara Del Mare-Roumani ${ }^{7}$, Rafael Valdes-Mas ${ }^{2}$, Yiming He ${ }^{2,3}$, Hodaya Karbi², Minhu Chen ${ }^{3}$, Alon Harmelin ${ }^{8}$, Ravid Straussman ${ }^{1}$, Nissan Yissachar $^{7}$, Eran Elinav ${ }^{2,9, \$}$, Benjamin Geiger ${ }^{1, \$}$

${ }^{1}$ Department of Molecular Cell Biology, Weizmann Institute of Science, Rehovot, 7610001, Israel

${ }^{2}$ Immunology Department, Weizmann Institute of Science, Rehovot, 7610001, Israel ${ }^{3}$ Department of Gastroenterology, The First Affiliated Hospital, Sun Yat-sen University, Guangzhou, China ${ }^{4}$ Department of Microbiology, Perelman School of Medicine, University of Pennsylvania, Philadelphia, PA, USA

${ }^{5}$ Institute for Immunology, Perelman School of Medicine, University of Pennsylvania, Philadelphia, PA, USA ${ }^{6}$ Department of Life Sciences Core Facilities, Weizmann Institute of Science, Rehovot, 7610001, Israel

${ }^{7}$ The Mina and Everard Goodman Faculty of Life Sciences ${ }^{1}$, Bar-Ilan Institute of Nanotechnology and Advanced Materials², Bar-Ilan University, Ramat-Gan, Israel, 5290002

${ }^{8}$ Department of Veterinary Resources, Weizmann Institute of Science, Rehovot, 7610001, Israel

${ }^{9}$ Cancer-Microbiome Research Division, DKFZ, Heidelberg, 69120, Germany

* These first authors contributed equally

\$ These last authors contributed equally

*All correspondence to:

Eran Elinav, M.D., Ph.D.

Immunology Department,

Weizmann Institute of Science,

234 Herzl Street,

Rehovot, Israel, 7610001

(08) 934-4014 (phone)

eran.elinav@weizmann.ac.il

Benjamin Geiger, Ph.D.

Department of Molecular Cell Biology

Weizmann Institute of Science,

234 Herzl Street,

Rehovot, Israel, 7610001

(08) 934-6111 (phone)

benny.geiger@weizmann.ac.il 


\section{Declaration of Interests}

E.E. is a consultant to DayTwo and BiomX. None of the topics related to this work involve these or other commercial entities. None of the other authors have any financial or non-financial competing interest.

\section{Author contributions}

I.G. and D.Z. designed, performed and interpreted the experiments, and wrote the manuscript. A.L., O.P., M.L., M.D-B., C.M., S.D.M-R., H.S., R.V-M., Y.H. and H.K. helped with experiments. O.G. developed the image quantification programs used here. N.Y., R.S., M.C. and A.H. provided essential tools and insights. E.E. and B.G. conceived the study, supervised the participants, interpreted the experiments, and wrote the manuscript.

\section{Acknowledgements}

We thank the members of the Elinav and Geiger labs, and members of the DKFZ cancer-microbiome division for excellent discussions and advice. We thank Dr. Noga Kozer and Dr. Haim Barr from the Wohl Drug Discovery team of the Nancy and Stephen Grand, Israel National Center for Personalized Medicine of the Weizmann Institute of Science, for their expert help with the design and execution of the high throughput screening described herein. We thank Dr. Richard Elliot and Dr. Chris Damen, Gates Foundation, for fruitful discussions. D.Z. is the recipient of the European Crohn's and Colitis Organization (ECCO) Fellowship, and is supported by the Ke Lin Program of the First Affiliated Hospital, Sun Yat-sen University. H.S. is supported by The V. R. Schwartz Research Fellow Chair. E. Elinav is supported by Yael and Rami Ungar; the Leona M. and Harry B. Helmsley Charitable Trust; Adelis Foundation; Pearl Welinsky Merlo Scientific Progress Research Fund; Lawrence and Sandra Post Family Foundation; Daniel Morris Trust; Park Avenue Charitable Fund; The Hanna and Dr. Ludwik Wallach Cancer Research Fund; Howard and Nancy Marks Charitable Fund; Aliza Moussaieff; Estate of Malka Moskowitz; Estate of Myron H. Ackerman; Estate of Bernard Bishin for the WIS-Clalit Program; Donald and Susan Schwarz; and by grants funded by the European Research Council; Israel Science Foundation; Israel Ministry of Science and Technology; Israel Ministry of Health; the Helmholtz Foundation; Else Kroener Fresenius Foundation; Garvan Institute; European Crohn's and Colitis Organization; Deutsch-Israelische Projektkooperation; and Welcome Trust. E. Elinav is the incumbent of the Sir Marc and Lady Tania Feldmann Professorial Chair; a senior fellow, Canadian Institute of Advanced Research (CIFAR); and an international scholar, The Bill \& Melinda Gates Foundation and Howard Hughes Medical Institute (HHMI). B.G. is the incumbent of the Erwin Neter chair in cell and tumor biology. 


\section{Abstract:}

Background \& Aims: The intestinal barrier protects intestinal cells from microbes and antigens in the lumen-breaches can alter the composition of the intestinal microbiota, the enteric immune system, and metabolism. We performed a screen to identify molecules that disrupt and support the intestinal epithelial barrier and tested their effects in mice.

Methods: We performed an imaging-based, quantitative, high-throughput screen (using CaCo- 2 and T84 cells incubated with lipopolysaccharide; tumor necrosis factor; histamine; receptor antagonists; and libraries of secreted proteins, microbial metabolites, and drugs) to identify molecules that altered epithelial tight junction (TJ) and focal adhesion morphology. We then tested the effects of TJ stabilizers on these changes. Molecules we found to disrupt or stabilize TJs were administered mice with dextran sodium sulfate-induced colitis or Citrobacter rodentium-induced intestinal inflammation. Colon tissues were collected and analyzed by histology, fluorescence microscopy, and RNA sequencing.

Results: The screen identified numerous compounds that disrupted or stabilized (after disruption) TJs and monolayers of epithelial cells. We associated distinct morphologic alterations with changes in barrier function, and identified a variety of cytokines, metabolites, and drugs (including inhibitors of actomyosin contractility) that prevent disruption of TJs and restore TJ integrity. One of these disruptors (putrescine) disrupted TJ integrity in ex vivo mouse colon tissues; administration to mice exacerbated colon inflammation, increased gut permeability, reduced colon transepithelial electrical resistance, increased pattern recognition receptor ligands in mesenteric lymph nodes, and decreased colon length and survival times. Putrescine also increased intestine levels and fecal shedding of viable $C$ rodentium, increased bacterial attachment to the colonic epithelium, and increased levels of inflammatory cytokines in colon tissues. Colonic epithelial cells from mice given putrescine increased expression of genes that regulate metal binding, oxidative stress, and cytoskeletal organization and contractility. Co-administration of taurine with putrescine blocked disruption of TJs and the exacerbated inflammation.

Conclusions: We identified molecules that disrupt and stabilize intestinal epithelial TJs and barrier function and affect development of colon inflammation in mice. These agents might be developed for treatment of barrier intestinal impairment-associated and inflammatory disorders in patients, or avoided to prevent inflammation.

KEY WORDS: cytokine, IBD, model, microbiota, leakage 


\section{Introduction}

Tightly regulated gut permeability is indispensable for the maintenance of intestinal homeostasis, healthy metabolism and immune tolerance. A single gut epithelial layer along the small and large intestinal mucosa allows for vital absorptive functions to co-exist with defense against an immense antigenic and microbial burden, introduced by food and the indigenous gut microbiota ${ }^{1}$. Intestinal barrier function consists of multiple components, mostly contributed by intestinal epithelial cell subsets, a robust junctional complex consisting of tight junctions (TJs) and adherens junctions (AJ), that form a physical barrier, reinforced by the cytoskeleton ${ }^{2}$, as well as secretion by goblet cells of mucus that separates the epithelial layer from the luminal microbiota ${ }^{3}$. Additionally, gut barrier is fortified by a complex and diverse mucosal immune system, in conferring tolerance against food and microbiota-derived antigens, while preserving an ability to elicit an intense immune response when the barrier is breached ${ }^{4,5}$.

At the sub-cellular and molecular levels, the intestinal barrier inter-cellular integrity is maintained by robust adhesions to their neighbors, and to the underlying extracellular matrix. Sealing of the epithelial monolayer is provided by the apical junctional complex, primarily by the TJs. TJs are comprised of multiple strands of adhesive transmembrane molecules (e.g. claudins, occludin, tricellulin, JAMs), connected via intracellular adaptor proteins (e.g. ZO-1, ZO-2, and ZO-3, cingulin) to the actin cytoskeleton ${ }^{6,7}$. At their basal aspects, TJs physically interact with and are mechanically reinforced by the cadherin-catenin-based $\mathrm{AJ}^{8}$. Focal adhesions (FAs) are cytoskeleton-associated multi-protein assemblies linking the cells, via integrin-mediated adhesions to the extracellular matrix ${ }^{9}$. Despite their crucial role in the epithelial biology, the specific molecular mechanisms, regulating and modulating TJ and FA integrity, remain poorly characterized ${ }^{10}$. Although current evidence shows that TJ proteins can be dynamically regulated by intracellular signaling transduction molecules such as small GTP-binding proteins, tyrosine kinases ${ }^{11}$, and extracellular stimuli such as bacteria, dietary component ${ }^{12}$, hyperglycemia ${ }^{13}$ and some cytokines ${ }^{11}$, the underlying regulatory mechanism is still far less elucidated to allow development of novel and potential therapeutic strategies targeting or protecting the intestinal barrier function.

A disruption of gut barrier integrity generates a "leaky gut", allowing an aberrant interaction of the luminal contents with the intestinal mucosal immune system. This process, when perpetuated in genetically susceptible individuals, leads to local dysregulation of immune responses, which culminates in chronic auto-inflammatory, leading to diseases such as inflammatory bowel disease $(I B D)^{14}$. In addition to IBD, pathogenesis of celiac disease ${ }^{15}$ or acute gut infectious diseases such as intestinal Citrobacter rodentium infection in mice ${ }^{16}$ also involves significant disruption of intestinal permeability. Influx of bacterial ligands into the portal and systemic circulation through leaky gut triggers systemic inflammation in a broad range of target organs. Gut leakiness is associated with a wide variety of extra-intestinal diseases, including systemic lupus erythematosus ${ }^{17}$, cardiometabolic disease $^{18}$, central nervous system disorders ${ }^{19}$ and aging-related disorders ${ }^{20}$. Although the direct cause and effect relationship between intestinal barrier dysregulation and these pathological states 
is not yet confirmed, reversing gut leakiness may become an attractive and potent target for disease prevention and treatment.

In this study, we sought to uncover new molecular regulators of intestinal intercellular and cell-matrix adhesions, as means of comprehensively elucidating the regulation of this important but elusive first line of defense. We opted to develop an automated microscopy-based high-throughput screening pipeline of molecules that directly target the epithelial barrier in vitro, using cultured cell lines, such as CaCo-2. Following validation and quantification of the effects on barrier integrity of known disruptors such as TNF, IL-1b, and bacterial LPS $21,22,23$ we studied the barrier-regulating function of molecules of dietary and microbiota origin, like taurine and histamine, previously shown to signal to gut epithelial cells ${ }^{24}$. We then widened our search to screen for thousands of barriermodulating compounds among a library of human secreted molecules containing cytokines, growth factors, hormones and other biologically active substances ${ }^{25}$, a metabolite library and a drug library. These screens led to the discovery of multiple TJ disruptors, as well as stabilizers, that blocked disruption, and even restored $\mathrm{TJ}$ integrity in affected CaCo-2 monolayers.

By using in vivo mouse models, we then validated that putrescine administration induced a 'leaky gut' phenomenon during both intestinal auto-inflammation and infection. Strikingly, coadministration of a stabilizer, taurine, significantly reversed the disruptive effect of putrescine on intestinal barrier function during enteric infection, suggesting that therapeutic application of novel epithelial barrier stabilizers may be explored as future means of preventing or treating infectious, metabolic and inflammatory human disease associated with gut barrier failure.

\section{Methods}

Cell culture: CaCo-2 (ATCC ${ }^{\circledR} \mathrm{HTB}-37$ ) and T84 (ATCC $\left.{ }^{\circledR} \mathrm{CCL}-248\right)$ cells were purchased from ATCC. Cells were routinely grown in DMEM tissue culture medium supplemented with $10 \%$ FCS, penicillin/streptomycin $100 \mathrm{U} / \mathrm{ml} / 0.1 \mathrm{mg} / \mathrm{ml}$, and $2 \mathrm{mM}$ GlutaMAX. For the experiments described herein, single-cell clones of the parental CaCo-2 population, displaying uniform TJ patterns, were isolated and used. For the screening, cells were seeded onto glass-bottom 96-well (Thermo Fisher) coated with either fibronectin or type I collagen (70,000 cells/well) and allowed to grow for 24 hours to form confluent monolayer before subjected to the indicated treatments. The same experimental design was also applied to T84 cells.

Imaging: Immunofluorescence images of manually-selected representative fields were acquired using DeltaVision Elite ${ }^{\circledR}$ wide field optical microscope (GE Healthcare) equipped with 40x/1.3 UPlanFLN or 60x/1.42 PlanApo $\mathrm{N}$ oil objective (Olympus). For multi-well plate scanning in screening experiments following automated imaging stations were used: DeltaVision microscope with plate scanning regiment, Hermes ${ }^{\circledR}$ imaging station (IDEA bio-Medical) or ImagExpress microXL imaging station (Molecular Devices) equipped with 40x air objectives. In all cases in order to cover the curvature of apical surface of epithelial monolayer $5 \mu \mathrm{m}$ thick z-stack was acquired around the TJs 
focus plane and the resulting image was generated as a maximum projection of all optical sections (Supplemental Figure 1A and B).

DSS-induced colitis: Mice were administered with $2 \%(\mathrm{w} / \mathrm{v})$ dextran sulfate sodium (DSS, M.W. = 36,000-50,000 Da; MP Biomedicals) in their drinking water for 5 or 7 days followed by regular water. For details please view the Supplemental methods.

Citrobacter rodentium infection: We used a kanamycin-resistant and luciferase-expressing derivative of Citrobacter rodentium DBS100 (ICC180) for infection as previously described ${ }^{13}$. For details please view the Supplemental methods.

\section{Results}

\section{Effects of known IBD-associated modulators on TJ and FA integrity in CaCo-2 cells}

A detailed description of the in vitro screening system is provided in the Supplemental Methods section. We started our evaluation of barrier function-modulating molecules by examining the effects of the known gut barrier modulators LPS and TNF on TJ and FA integrity, by treating cultured ZO-1 and zyxin- co-labeled CaCo-2 cells for 24 hours with either molecule. While the TJs of nontreated cells remained intact (Figure 1A), both LPS and TNF induced an apparent deformation of TJs, manifested by tortuous, often discontinuous morphology, which we refer to as "Type-I disruption" (Figure 1B, C). To quantify these effects, we measured the junctions' tortuosity and found that, while the vast majority of junctions were straight and intact in non-treated cells (tortuosity value 1.14+/0.11), LPS and TNF treatment resulted in significant increase of junction distortion, manifesting as tortuosity values of $1.46+/-0.31$ and $1.41+/-0.31$, respectively (Figure 1I). Interestingly, labeling of FAs revealed that treatments with both molecules resulted in significant increase of FA length (axial ratio values of $4.00+/-0.88$ and $3.42+/-0.73$, instead of $2.66+/-0.61$ in control, Figure 1 A, B, C, right panels, quantified in Figure 1J). Interestingly, the inflammatory cytokine IL-1 $\beta$ induced a TJ distortion similar to that induced by LPS and TNF, while its effect on FAs was limited (Figure 1D, quantified in Figure 1I, J, respectively). The specificity of the IL-1 $\beta$ effect on TJ morphology was validated using a receptor antagonist (IL-1ra), which completely suppressed the IL-1 $\beta$-induced TJ distortion (Figure 1E, quantified in Figure 1I).

We then tested the effect on TJ integrity of histamine, the microbiota-associated metabolite previously shown to be highly abundant in the feces of dysbiotic mice and to increase colitis severity in mice ${ }^{24}$. Unlike the Type-I distortive effects of LPS, TNF and IL-1 $\beta$, histamine treatment induced a dramatic change of TJ, as manifested by a major deformation of TJ geometry, leading to the development of multiple indentations and protrusions (Figure 1F). We named this phenotype "Type-II disruption". Morphometrical quantification by "TJ roughness" (See Supplemental methods) showed a remarkably increased TJ roughness, induced by histamine (Figure 1K). An addition of Ranitidine, a specific antagonist of $\mathrm{H} 2$ histamine receptor known to be expressed in the gastrointestinal tract ${ }^{26}$, significantly reduced the disruptive effect of histamine on TJ morphology 
(Figure 1G, quantified in Figure 1K), while antagonists of other histamine receptors (e. g. H1 antagonist desloratadine) were not able to block this effect (Figure 1H). Similar to Type-I disruptors, Type-II disruptors also had a major effect on FA dimensions, manifested by an apparent increase in FA area (Figure 1F) as well as a measurable increase in FA axial ratio (Figure 1J). $\mathrm{H} 2$ antagonists efficiently abolished histamine-induced increase of FAs, while $\mathrm{H} 1$ antagonist did not (Figure 1G, H right panels).

To obtain a comprehensive 3-dimensional (3D) characterization of TJ deformation, in the whole cell context, we conducted a 3D deconvolution reconstruction microscopy of CaCo-2 cells treated with LPS or histamine and labelled for ZO-1 and F-actin or b-catenin. While untreated cells featured a normal columnar morphology (Figure 2A), the Type-II phenotype induced by histamine was shown by $3 \mathrm{D}$ reconstruction to affect primarily the apical cell domain (Figure $2 \mathrm{~B}$ ), but not impacting the rest of cell body. Interestingly, LPS treatment resulted in impacts noted on TJ integrity along the "apical junctional belt" in the same cell, including relatively intact junctional regions, distorted TypeI regions and visibly fragmented areas (Figure 2C, enlarged view in insets 1, 2 and 3, respectively). The level of TJ distortion correlated with organization of apical F-actin, with intact areas uniformly decorated by F-actin, the Type-I regions characterized by interrupted, punctuate actin labeling while the fragmented areas completely losing actin association (Figure $\mathbf{2 C}$ ). A substantial uncoupling between TJ and AJ was demonstrated by double-labeling for TJs (anti ZO-1) and AJ (anti b-catenin), which are the two canonical components of the epithelial junctional complex, as an overlay of apical ZO-1 labeling and sub-apical b-catenin staining showed (Figure 2D).

In all, by testing known auto-inflammation-associated molecules, we confirmed that the in vitro microscopy-based system is reliable for monitoring TJ and FA integrity in CaCo-2 cells, and characterized two distinct morphological variants of TJ disruption, thus enabling high-throughput screening and assessment of molecules involved in the regulation of intestinal barrier function.

\section{High throughput screening for novel TJ and FA modulators in CaCo-2 cells}

Based on the effect of aforementioned inflammatory mediators on TJ and FA organization, we expanded to search for novel modulators of intestinal barrier function, by screening three different molecular libraries.

a. A human secreted molecule ('secretome") library. This library is composed of a variety of human-secreted molecules containing cytokines, chemokines, growth factors, hormones, enzymes and other biologically-active molecules ${ }^{25}$. Out of 298 molecules included in the library, we identified 10 which induced typical Type-I appearance of TJs (i.e. increasing junctional tortuosity), and included cytokines (IL-15), chemokines (CCL-20, CCL-23), growth factors (FGF-1, FGF-10, BMP-10), neuropeptides (UTS-2, UCN-1, UCN-3) and the hormone erythropoietin (Supplemental Figure 2A, C and Supplemental Table 1). Essentially all TJ disruptors discovered in this screen increased FA length and area (Supplemental Figure 2A, D). We also identified 4 molecules that significantly improved TJ integrity, referred to as TJ stabilizers, including IL-21, CCL-3, TIMP-2 and FasLG (Supplemental Figure 2B, C). In addition to improvement of steady-state TJ morphology, these epithelial stabilizers 
possessed broad capacity to prevent TJ disruption caused by disrupting agents of different origins, as discussed below.

b. A microbiota-associated metabolite library. Despite the high variety (in the thousands) and concentrations (in the millimolar range) of many microbiota-associated metabolites at the hostmicrobiota interface, only a few, including taurine ${ }^{24}$, butyrate ${ }^{27}$ and microbial-specific indoles ${ }^{28}$, have been reported to modulate gut permeability. To identify novel modulating metabolites, we screened 25 molecules previously identified to be differentially abundant in healthy and dysbiotic mice (deficient in the inflammasome adaptor $\mathrm{ASC}^{24}$ ), based on their abundance, stability and water solubility (Supplemental Table 2). We identified three potential disruptive metabolites (acetylproline, spermine and putrescine) and three stabilizing metabolites (taurine, tryptamine and Lhomoserine) (Supplemental Results, Supplemental Figure 3).

c. An FDA-approved drug library. This library contains 2956 pharmacologically active compounds. Screening identified over 80 TJ stabilizers and disruptors, among them cell cycle and transcriptional modulators, kinase inhibitors, retinoic acid derivatives, specific flavonoids and more (Supplemental Results, Supplemental Figure 4 and Supplemental table 3)

\section{Epithelial stabilizers are dominant over multiple disruptors in restoring $\mathrm{TJ}$ integrity}

Following the identification of multiple TJ stabilizers that suppress the effects of specific disruptors, we assessed whether these stabilizers are specific only for a single disrupting agent, or feature a broad stabilizing capacity against multiple TJ-disrupting molecules of different nature and origins. To this aim, we treated CaCo-2 cells with multiple disruptors (of secretome, metabolite and pharmaceutical library origins) in combination with multiple stabilizers of diverse origins, and checked the cellular response in all combinations. Collectively, the tested stabilizing molecules commonly demonstrated a broad capacity to prevent TJ disruption, irrespective of the nature or signaling properties of the disrupting agent. As shown in Figure 3A-C disruption caused by either inflammatory cytokine IL-15 or intestinal metabolites histamine and putrescine could be blocked by same subset of stabilizing agents. Quantifications performed for all tested combinations revealed that diverse modes on TJ disruption could be prevented by tested stabilizers, though the degree of stabilization varied among compounds (Figure 3D). Importantly, not all stabilizers were capable of preventing Type-II disruption, induced by histamine and spermine (Figure 3C, D). Capacity of certain stabilizers to prevent disruption induced by multiple agents of different origin is an intriguing feature due to the apparent molecular diversity of the disruptors and their distinct biological activities (via cytokine receptors, growth factor receptors, histamine receptors, primary effects on cell cycle, cytoskeleton, signaling machinery etc.). It suggests the possibility of universal downstream executive mechanism of TJ disruption triggered via different pathways and ability of stabilizing agents to effectively neutralize this mechanism. One example of such mechanism is further discussed below.

We have further demonstrated that epithelial stabilizers are capable not only of blocking disruption, when administered simultaneously with different disruptors, but also of restoring TJ integrity in cells 
displaying a pre-existing disrupted phenotype (Supplemental Results and Supplemental figure 5). To verify that these intestinal barrier function-modifying activities were not cell-line specific, we tested the effects of some newly-identified disruptors and stabilizers, using the T84 cell line of gastrointestinal origin, which serves as a common model for assessing the epithelial barrier function ${ }^{29}$. Results showed that treatment of T84 cells with Type-I disruptor (LPS) or with Type-II disruptors (histamine, putrescine) resulted in TJ disruption, comparable to that observed in CaCo-2 cells, and in both cases, the deterioration of TJs was completely blocked by both taurine and ATRA (Supplemental Results and Supplemental Figure 6).

We next assessed whether the dominant stabilizing capacity displayed by multiple molecules was also apparent using more complex physiologically-relevant metabolite mixtures. To this aim, we tested the modulatory functions of aqueous fecal extracts from healthy mice and dysbiotic (Asc-/-) mice at our vivarium ${ }^{30}$. Addition of fecal extract from healthy animals to regular culture medium did not induce detectable changes in TJ integrity (Figure 4A). In contrast, fecal extracts from Asc-/-mice, caused a severe Type-I disruption, similar to that observed upon treatment with multiple disrupting agents. Strikingly, such TJ deformation was significantly reduced by co-addition of fecal extracts from healthy mice, indicating that feces from healthy mice contain stabilizing components that can protect TJs from disruptors present in the feces of dysbiotic mice. We further tested the protective function of healthy fecal extracts against specific disruptive agents, and noted that TJ alterations induced by LPS or histamine can be prevented by co-treatment with the fecal extracts from healthy mice. In addition, disruptive effects on TJs caused by the fecal extracts from dysbiotic mice could be prevented by co-treatment with the stabilizing metabolites taurine or tryptamine (Figure 4A).

To validate that these dominant stabilizing effects on TJ morphology also impact transmembrane permeability, we measured the trans-epithelial electrical resistance (TEER) and permeability of fluorescent dextran across the CaCo-2 monolayer. Treatment with the various disruptors caused a major drop in the TEER and elevation of the permeability of dextran, reflecting an intestinal barrier functional alteration paralleling the morphological disruption. Co-treatment with taurine abolished these functional barrier defects (Figure 4B-D). Interestingly, bacterial LPS caused TEER decrease but had limited effect on dextran leakage, suggesting that structural distortion of TJs may differentially affect permeability to ions and macromolecules.

Taken together, these data suggest that multiple simultaneously or sequentially administered purified stabilizers or those present in fecal extracts from healthy mice may reverse, the morphologically and functionally disrupted intestinal barrier induced by purified disruptors or those present in fecal extracts of dysbiotic mice.

\section{A role for acto-myosin contractility in the regulation of $\mathrm{TJ}$ integrity}

We next utilized our in vitro system in seeking a molecular mechanism of exemplary compounds impacting intestinal barrier function. It was previously shown that the activation of acto-myosin contractility via phosphorylation of myosin regulatory light chain by myosin light chain kinase (MLCK) can induce TJ disruption and increase epithelial permeability ${ }^{31}$. In order to examine whether 
this mechanism may explain the function of some of the epithelial disruptors identified in our screens, we tested the ability of the acto-myosin contractility inhibitor, blebbistatin, to prevent TJ disruption using the hallmark type-I disruptor LPS, or the hallmark type-II disruptor histamine. Indeed, co-treatment of CaCo-2 cells with blebbistatin abolished the TJ disruption induced by the LPS or histamine alone (Figure 5A). Interestingly, the enlargement of FAs induced by the disruptors was also blocked by blebbistatin (Figure 5A). In addition to type II disruption of TJ, histamine treatment induced the formation of robust radial F-actin bundles at the apical cell domain, which were not formed under untreated conditions (Figure 5B). Thus, the morphology of apical actin fibers upon treatment with disruptors suggests increased contractility applied to apical junctional complex, which apparently leads to histamine-induced TJ deformation.

To further test the effect of selected epithelial disruptors and stabilizers on cellular contractility we employed traction force microscopy, a method allowing to microscopically measure contractile forces applied by cells to the underlying substrate ${ }^{32}$. Indeed, this analysis revealed an increased contractility upon treatment of $\mathrm{CaCo}-2$ cells with the disruptive agent histamine and almost complete cellular relaxation upon treatment with the stabilizers tryptamine and blebbistatin (Figure 5C upper panel). Another strong disruptor, putrescine, caused strong contraction of epithelial islands. In order to directly assess whether disruptor-induced contraction can be reversed by the presence of stabilizers we co-incubated cells with putrescine and three stabilizers (taurine, tryptamine and ATRA). Indeed, taurine significantly reduced putrescine-induced contraction to the level similar to control, while tryptamine and ATRA caused even more significant relaxation, reducing the contractility of co-treated cells below the control level (Figure 5C bottom panel). We further confirmed the effect of acto-myosin contractility on TJ integrity using pharmacological activator of acto-myosin contractility Calyculin A and assessing its effect on TJ morphology (Supplemental results and Supplemental Figure 7).

\section{In vivo validation of a novel metabolite disrupting intestinal barrier function}

Based on the observed effect of the aforementioned TJ modulators, we sought to provide ex vivo and in vivo validation of an exemplary novel metabolite, putrescine, one of the most powerful TJ disruptors identified in our in vitro screen. To assess the effect of putrescine on steady-state intestinal barrier function, we implemented an ex vivo three-dimensional colon culture system which can preserve the tissue architecture and mimic in vivo conditions ${ }^{33}$. Media containing different concentrations of putrescine were perfused continuously through the gut lumen for 2 hours (Supplemental Figure $\mathbf{8 A}$ ). We detected dose-dependent histological damages of colon tissues, as manifested by gradual epithelial detachment and crypt loss as the concentration of putrescine increased (Supplemental Figure 8B). Notably, at low putrescine concentrations of as low as $33.2 \mathrm{mM}$, in which only mild tissue damage was observed and only mild histological changes were documented, we could nonetheless detect a significantly decreased ZO-1 intensity as compared to controls (Figure 6A, B, Supplemental Figure 8C), indicating that putrescine treatment disrupted TJ integrity in steady state colon tissue, even in the absence of massive tissue damage. 
To further characterize the in vivo effect of putrescine, we administered putrescine to naïve wildtype mice by oral gavage for 10 days, followed by induction of experimental colonic inflammation by Dextran sulfate sodium (DSS) ${ }^{34}$, and by $C$. rodentium infection, the later simulating human enteropathogenic Escherichia coli infection and associated large intestinal inflammation ${ }^{16}$. Both models are known to physically and functionally impair intestinal barrier and corroborate some histo-pathological features of human IBD. We began by investigating the potential of putrescine to exacerbate intestinal barrier function before the onset of overt intestinal inflammation, tissue damage and systemic manifestations. To this aim, mice were exposed to DSS for 5 days, when no significant weight loss was yet observed (Supplemental Figure 8D, E). Compared with vehicletreated mice, putrescine-administered mice featured enhanced gut permeability, as indicated by an increased systemic FITC-dextran influx (Figure 6C), a higher electrical short-circuit current (Figure 6D) as well as a lower colonic trans-epithelial electrical resistance (Figure 6E). As another readout of local gut leakiness, we detected increased amounts of microbial pattern recognition receptor ligands in the mesenteric lymph nodes in putrescine-treated mice (Figure 6F), and to a lesser extent in the spleen or liver (Supplemental Figure 8F, G). During the inflammatory stage of DSS-induced colitis, putrescine-treated mice featured enhanced disease severity compared to vehicle-treated mice, despite comparable intake of DSS during treatment (Supplemental Figure 9A, B). Increased putrescine-induced disease burden manifested as exacerbated weight reduction, significantly lower survival, enhanced colonoscopy severity score, decreased colon length, and aggravated histopathological severity (Supplemental Figure 9C-J). We next assessed whether supplementation of a newly discovered TJ stabilizer could rescue the disruptive effect of putrescine in vivo. To this aim, mice were co-administered with 3\% taurine in drinking water and putrescine by oral gavage for 10 days, followed by DSS treatment. The susceptibility to DSS colitis in putrescine-treated mice could be partially but significantly ameliorated by co-administration of taurine, characterized by less weight loss (Figure 6G), lower mortality rate (Figure $\mathbf{6 H}$ ), increased colon length (Figure 6I) and improved histopathological severity (Figure 6J, K).

Likewise, putrescine treatment of mice infected with a bioluminescent variant of $C$. rodentium exacerbated disease, as noted by higher bacterial burden in the colon and systemic sites, more disruptive intestinal barrier function and increased levels of inflammatory cytokines (Supplemental results and Supplemental Figure 10,11). Addition of taurine partially but significantly restricted the susceptibility to $C$. rodentium infection in putrescine-treated mice, characterized by lower bacterial shedding in feces (Figure 7A) and lower bacterial bioluminescence (Figure 7B, C). Taurine supplementation also ameliorated putrescine-induced colonic bacterial attachment (Figure 7D-F) and systemic dissemination to spleen, liver and mesenteric lymph nodes (Figure 7G-I), accompanied by significantly lower electrical current recording and higher trans-epithelial electrical resistance noted in intestinal epithelial cells under this stabilizing treatment (Figure 7J, K).

To assess the effects of putrescine and taurine treatment on sorted EpCAM+ colonic epithelial cells of mice, we performed RNA-sequencing analysis on these cells, showing a possible rescuing effect of taurine in the oxidative stress and cellular contractility caused by putrescine (Supplemental 
results and Supplemental Figure 12). Taken together, our ex vivo and in vivo data validated that, while the novel TJ-disrupting metabolite putrescine contributes to impaired intestinal barrier function, aggravates gut leakiness and subsequently disease susceptibility during colonic autoinflammation and infection, co-supplementation with the stabilizer taurine may enable to significantly reverse this disruptive effect.

\section{Discussion}

We demonstrate that a variety of compounds derived from the host, microbiota, or from screening libraries can modulate the structural integrity of TJs and FAs in intestinal epithelial cells, thereby affecting their barrier functionality. Using cultured CaCo-2 cells as a screening system and TJ morphology as our primary readout, we have identified multiple secreted molecules (including inflammatory cytokines) and microbial-associated metabolites, that disrupt the integrity of TJs. This structural effect was manifested either by increased TJ tortuosity (Type-I disruptive effect), or by gross perturbation of TJ topology throughout the epithelial monolayer (Type-II disruptive effect). One of the characteristic structural features of both Type-I and Type-II effects was the radical disarray of the inter-cellular apical junctional complex. This complex consists of a apical belt-like structure, known as the "occludens", or "tight junction", which controls the apical-basal polarity of the cells, as well as the trans-epithelial permeability ${ }^{35,36}$, and a closely-associated sub-apical belt, known as 'adherens junction', which is a robust, actin-rich, cadherin-based cell-cell adhesion, that is believed to mechanically stabilize the adjacent $\mathrm{TJ}^{37,38}$. As shown here, in control cells, the two junctions are, indeed, closely associated (Figure 2D), yet both modes of disruption lead to major disengagement of the two. The molecular 'targets' of the various disruptors, and how the disengagement of the junctional components leads to the loss of barrier integrity remains to be determined in future studies. Moreover, our functional validation experiments confirmed that these structural effects are accompanied by a considerable decrease of trans-epithelial resistance and increase in dextran permeability in the treated cells (Figure 4C, D) both in in vitro and in vivo experiments.

Our findings bear several implications. First, we demonstrate that intestinal barrier function is a highly regulated and dynamic process, which is influenced by multiple 'environmental players' from both host and microbiota origin, which include the junction modulators discovered herein, as well as yet to be discovered physiological, pathological or pharmacological effectors of epithelial intercellular adhesions. Such critical gate-watcher regulation, which may have critical consequences on the host's anti-pathogen protection ${ }^{39}$, as well as its metabolic and immune homeostasis ${ }^{40,3}$, must react to a combination of signals which integrate to accurately reflect a changing microenvironment. Using our high-throughput screening system we demonstrate that these assorted signals come from both the host and microbiota, highlighting further the concerted mucosal behavior of the prokaryotic and eukaryotic components of the holobiont ${ }^{41}$.

Second, our study provides proof-of-concept to new prototypes of gut epithelial cell molecular regulation of TJ and FA integrity, and their biochemical basis. Indeed, a cross-talk between the apical 
junctional complex of epithelial cells (including the TJs and AJ) and FAs was demonstrated in multiple systems, suggesting that increased contractility generated by the actomyosin system, stimulate FA formation and growth, and, at the same time, has a detrimental effect on cell-cell junctions ${ }^{42,43}$. The results described herein, indicating that nearly all TJ disruptors augmented FA formation (Figure 1), while most stabilizers exerted the opposite effect, strongly support these findings, and indicate that the inter-adhesion cross-talk may be important for maintaining the gut homeostasis. We further show here that mechanical forces play an important role in TJ/FA regulation. Thus, selected prominent disruptors (e.g. Histamine, Putrescine Figure $\mathbf{5 C}$ ), stimulate cell contractility and subset of epithelial stabilizers prevented this effect, as measured by traction force microscopy. In addition, a classical actomyosin contractility blocker, blebbistatin, blocks the effect of all tested disruptors (Figure 5A). This is also in line with previous reports, suggesting that the activation of myosin II, by myosin light chain kinase correlates with increased trans-epithelial permeability in the gut ${ }^{44,45}$. The disruptive effect of excessive contractility was further confirmed, showing that the phosphatase inhibitor calyculin, which enhances actomyosin contractility (Supplemental figure 7A), act as a strong and rapidly-acting disruptor, whose activity can be blocked by pretreatment with different stabilizers (Supplemental figure 7B). The differential (largely - inverse) effects of disruptors on the apical adhesions (cell-cell) and basal adhesions (cell-matrix), can bear relevance to intestinal epithelial biology. Naturally, TJ instability can play key and direct roles in the penetration of lumenal micbobiota across the epithelium, and initiating an inflammatory response, and adhesion to the underlying matrix plays a major role in reinfircing the gut wall integrity.

Third, our study highlights the potential physiological importance of this complex regulatory network on mammalian physiology and microbiota-associated disease. Our results support the paradigm that an aberrant combination of 'disrupting signals', or loss of 'stabilizing signals' from the host and its microbiota, coupled with genetic susceptibility for such barrier instability, may drive the system towards pathological intestinal barrier function, in a variety of disorders such as IBD ${ }^{46}$, cardiometabolic disease ${ }^{47}$, infectious mucosal disorders ${ }^{13}$, and even mental illness ${ }^{48}$. In face of genetic susceptibility, such 'common denominator' intestinal barrier function-disrupting initiating event may drive an aberrant contact between foreign elements in the form of food- and microbiotaassociated molecules and the mucosal immune arm, triggering a perpetuated and self-amplifying immune response which contributes to disease development, and further augment barrier dysfunction. Importantly, our results suggest that many of the new 'barrier stabilizers' discovered in this study may dominantly act when co-administered with disruptors in restoring barrier integrity. As such, the stabilizers increase the apparent cohererence of TJs in untreated cells, and, more importantly, they effectively block the disruptor effects, irrespective of their molecular origin or expected mode of action. For example, the TJ disruption by histamine, can be specifically inhibited by the type 2 histamine receptor blocker Ranitidine, yet it can also be inhibited by several other stabilizers, which are not expected to have any effect on histamine receptor signaling (Figure 1 and Figure 3). This apparent dominance of TJ stabilizers' effect over disruptors is unique for the physiological and pathological gut molecular ecosystem, and suggests that a combination of 
stabilizing molecules and even drugs may enable to contain this chain of events upstream to uncontrollable inflammation, as means of ameliorating microbiota- and food antigen-associated disease.

\section{Figure legends}

Figure 1. Inflammatory IBD mediators affect TJ and FA integrity in cultured CaCo-2 cells. CaCo-2 cell were incubated in regular medium (A), treated with $300 \mathrm{ng} / \mathrm{ml}$ LPS (B), 50ng/ml TNF (C), 30ng/ml IL-1 $\beta$ (D) or 10mM histamine (F). IL1- $\beta$ and histamine were combined with their respective inhibitors,

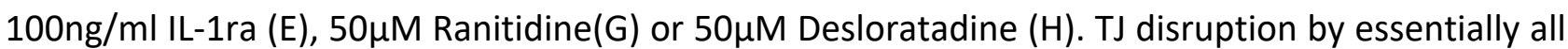
Type-I disruptors was quantified by calculating its tortuosity (I); FA elongation (quantified by axial ratio calculation) was used for quantifying FA deformation (J); TJ roughness was measured for calculating the effect of histamine (Type-Il disruptor) (K).

Figure 2. Type-I and type-II disruptors uncouple TJs from AJs at the apical cell domain. CaCo-2 cells untreated $(A)$ or treated for 24 hours with $10 \mathrm{mM}$ histamine (B) were fixed-permeabilized and double-labeled for cingulin (TJ) and $\beta$-catenin (AJ), both shown in red, TJ channel pseudocolored. Confocal stack covering the entire thickness of monolayer is shown here as a z-projection (left panel) or a tilted side-view (middle panel). 3D rendering was performed for the reconstruction of the TJ-AJ relationships (right panel). The arrow and arrowhead point to the same protrusions in histamine treated cells, asterisks indicate the control cells used for the 3D rendering. (C) The apical domain of LPS-treated cells (300 ng/ml), labeled for ZO-1 to visualize TJs (upper left and pseudocolored in yellow) and with phalloidin to visualize AJ-associated actin (middle panel and pseudocolored in light blue). (D) The apical and sub-apical aspects of untreated CaCo-2 cells (left panel), cells treated with LPS (center) or histamine (right).

Figure 3. TJs stabilizers can effectively block the effects of multiple disruptors. CaCo-2 cells were treated for 24 hours with different disruptor-stabilizer combinations, fixed-permeabilized and labeled for ZO1 to visualize TJs. (A-C) Selected disruptive molecules (IL-15, putrescine, histamine) were combined with stabilizers derived from human secreted molecules, bacterial metabolites and pharmacological agents (IL-21, tryptamine, ATRA). (D) TJ disruption was quantified for 14 disruptors causing Type-I or Type-II phenotypes in combinations with 8 major TJ stabilizers, identified in human secretome library, bacterial metabolites library and a pharmacological stabilizer (ATRA). Junctional tortuosity (left panel) and roughness (right panel) were calculated for all combinations, displaying Type-I or Type-II phenotype, respectively. The heat-maps present $\Delta$ tortuosity and $\Delta$ roughness values.

Figure 4. (A) Healthy and dysbiotic fecal extracts act as TJ stabilizers and disruptors, respectively. (B-D) Epithelial disruptors/stabilizers differentially affect barrier function in vitro. 
(A) Aqueous extracts of feces of healthy (wt) or dysbiotic (Asc-/-) mice were applied to cultured CaCo-2 cells individually, together or in combination with specific TJ disruptors and stabilizers. (B) CaCo- 2 cells were treated with indicated disruptors alone or in combination with taurine, fixed and stained for ZO1 to visualize TJs. (C, D) The effects of selected epithelial disruptors, alone and in combination with taurine, on trans-epithelial resistance (TEER, C) and dextran permeability (D) in CaCo-2 monolayer.

Figure 5. Modulation of acto-myosin contractility is involved in TJ disruption and stabilization processes. (A) CaCo- 2 cells were treated for 24 hours with LPS or histamine alone or in combination with the acto-myosin contractility blocker blebbistatin, fixed and labeled for the TJ component cingulin and the FA molecule paxillin. (B) CaCo-2 cells untreated or treated with histamine for 24 hours were fixed and labeled for ZO-1 and phalloidin. Actin was imaged in the same focal planes as ZO-1 to determine the apical organization of the actin cytoskeleton (ZO-1 pseudo colored in yellow and actin in blue). (C) CaCo-2 cells were plated as individual islands on type-I collagen-coated elastomeric hydrogel and treated for 24 hours with either individual disruptor (histamine, putrescine) or stabilizer (taurine, ATRA, tryptamine) or with the contractility inhibitor blebbistatin or with disruptor/stabilizer mixtures. Fluorescent beads embedded in the hydrogel were imaged before and after cell removal from the substrate. Beads displacement was used for calculating the traction forces applied by the cells to the underlying matrix (presented as heat map, with scale above the panel).

Figure 6. Disruptive effect of putrescine ex vivo and in mice with DSS-induced colitis. Representative histology images (A) and quantification of ZO1 intensity (B) in colon tissues cultured with control medium or putrescine $(1: 300,33.2 \mathrm{mM})$. Wild-type mice with oral gavage of putrescine or vehicle control (PBS) were administered with 2\% DSS in the drinking water for 5 days (C-F) or 7 days (G-K) ( $n=8-10$ mice per group). FITC-dextran levels recovered from the serum 3 hours after oral gavage ( $80 \mathrm{mg} / \mathrm{ml}$ FITC-dextran) (C), Ussing chamber recording of short circuit current (ISC) (D) and trans-epithelial electrical resistance (TEER) across colon epithelial layer (E) and pattern recognition receptor stimulation by lymph node extracts from mice (F) on day 5 after DSS administration. Weight loss $(G)$, survival $(H)$, measurement of colon length $(I)$, representative histology images $(J)$, and pathology scoring on day 12 after DSS treatment (K). Ctrl: control medium; PUT: putrescine; TAU: taurine.

Figure 7. Restoration of the disruptive effect of putrescine by taurine supplement in mice with $C$. rodentium infection. Wild-type mice treated with PBS, putrescine, putrescine plus taurine were infected with $C$. rodentium (results were pooled from 3 independent experiments). (A-C) Colonyforming units (CFUs) recovered from stool (A), abdominal bioluminescence quantification (B) and imaging (C) during the post-infection course. (D-F) CFUs recovered from colonic tissue (D), ex vivo colonic bioluminescence quantification (E) and imaging (F) and on day 7 post infection. (G-H) CFUs 
recovered from spleens $(\mathrm{G})$, livers $(\mathrm{H})$ and lymph nodes $(\mathrm{I})$ on day 7 post infection. (J-K) Ussing chamber recording of short circuit current (ISC) (J) and trans-epithelial electrical resistance across colon epithelial layer (K) on day 7 post infection. PUT: putrescine; TAU: taurine.

\section{References}

1. France, M. M. \& Turner, J. R. The mucosal barrier at a glance. J. Cell Sci. 130, 307-314 (2017).

2. Turner, J. R. Intestinal mucosal barrier function in health and disease. Nat. Rev. Immunol. 9, 799-809 (2009).

3. Peterson, L. W. \& Artis, D. Intestinal epithelial cells: regulators of barrier function and immune homeostasis. Nat. Rev. Immunol. 14, 141-153 (2014).

4. Hooper, L. V. \& Macpherson, A. J. Immune adaptations that maintain homeostasis with the intestinal microbiota. Nat. Rev. Immunol. 10, 159-169 (2010).

5. Roy, U. et al. Distinct Microbial Communities Trigger Colitis Development upon Intestinal Barrier Damage via Innate or Adaptive Immune Cells. Cell Rep. 21, 994-1008 (2017).

6. Tsukita, S., Furuse, M. \& Itoh, M. Multifunctional strands in tight junctions. Nat. Rev. Mol. Cell Biol. 2, 285-293 (2001).

7. Furuse, M. Molecular Basis of the Core Structure of Tight Junctions. Cold Spring Harb. Perspect. Biol. 2, a002907-a002907 (2010).

8. Campbell, H. K., Maiers, J. L. \& DeMali, K. A. Interplay between tight junctions \& adherens junctions. Exp. Cell Res. 358, 39-44 (2017).

9. Geiger, B., Spatz, J. P. \& Bershadsky, A. D. Environmental sensing through focal adhesions. Nat. Rev. Mol. Cell Biol. 10, 21-33 (2009).

10. Odenwald, M. A. \& Turner, J. R. The intestinal epithelial barrier: a therapeutic target? Nat. Rev. Gastroenterol. Hepatol. 14, 9-21 (2017).

11. Chelakkot, C., Ghim, J. \& Ryu, S. H. Mechanisms regulating intestinal barrier integrity and its pathological implications. Experimental and Molecular Medicine 50, 103 (2018).

12. Ulluwishewa, D. et al. Regulation of Tight Junction Permeability by Intestinal Bacteria and Dietary Components. J. Nutr. 141, 769-776 (2011).

13. Thaiss, C. A. et al. Hyperglycemia drives intestinal barrier dysfunction and risk for enteric infection. Science (80-. ). 359, 1376-1383 (2018).

14. De Souza, H. S. P. \& Fiocchi, C. Immunopathogenesis of IBD: Current state of the art. Nature Reviews Gastroenterology and Hepatology 13, 13-27 (2016).

15. Schumann, M., Siegmund, B., Schulzke, J. D. \& Fromm, M. Celiac Disease: Role of the Epithelial Barrier. CMGH 3, 150-162 (2017).

16. Collins, J. W. et al. Citrobacter rodentium: Infection, inflammation and the microbiota. Nature Reviews Microbiology 12, 612-623 (2014). 
17. Mu, Q., Kirby, J., Reilly, C. M. \& Luo, X. M. Leaky gut as a danger signal for autoimmune diseases. Frontiers in Immunology 8, 598 (2017).

18. Winer, D. A., Luck, H., Tsai, S. \& Winer, S. The intestinal immune system in obesity and insulin resistance. Cell Metabolism 23, 413-426 (2016).

19. Köhler, C. A. et al. The Gut-Brain Axis, Including the Microbiome, Leaky Gut and Bacterial Translocation: Mechanisms and Pathophysiological Role in Alzheimer's Disease. Curr. Pharm. Des. 22, 6152-6166 (2016).

20. Thevaranjan, N. et al. Age-Associated Microbial Dysbiosis Promotes Intestinal Permeability, Systemic Inflammation, and Macrophage Dysfunction. Cell Host Microbe 21, 455-466.e4 (2017).

21. Ma, T. Y. et al. TNF- $\alpha$-induced increase in intestinal epithelial tight junction permeability requires NF-kB activation. Am. J. Physiol. Liver Physiol. 286, G367-G376 (2004).

22. Al-Sadi, R. M. \& Ma, T. Y. IL-1beta causes an increase in intestinal epithelial tight junction permeability. J. Immunol. 178, 4641-9 (2007).

23. Guo, S., Al-Sadi, R., Said, H. M. \& Ma, T. Y. Lipopolysaccharide Causes an Increase in Intestinal Tight Junction Permeability in Vitro and in Vivo by Inducing Enterocyte Membrane Expression and Localization of TLR-4 and CD14. Am. J. Pathol. 182, 375-387 (2013).

24. Levy, M. et al. Microbiota-Modulated Metabolites Shape the Intestinal Microenvironment by Regulating NLRP6 Inflammasome Signaling. Cell 163, 1428-1443 (2015).

25. Shee, K. et al. Therapeutically targeting tumor microenvironment-mediated drug resistance in estrogen receptor-positive breast cancer. J. Exp. Med. 215, 895-910 (2018).

26. Parsons, M. E. \& Ganellin, C. R. Histamine and its receptors. Br. J. Pharmacol. 147, S127-S135 (2009).

27. Plöger, S. et al. Microbial butyrate and its role for barrier function in the gastrointestinal tract. Ann. N. Y. Acad. Sci. 1258, 52-59 (2012).

28. Venkatesh, M. et al. Symbiotic Bacterial Metabolites Regulate Gastrointestinal Barrier Function via the Xenobiotic Sensor PXR and Toll-like Receptor 4. Immunity 41, 296-310 (2014).

29. Devriese, S. et al. T84 monolayers are superior to Caco-2 as a model system of colonocytes. Histochem. Cell Biol. 148, 85-93 (2017).

30. Elinav, E. et al. NLRP6 inflammasome regulates colonic microbial ecology and risk for colitis. Cell 145, 745-757 (2011).

31. Cunningham, K. E. \& Turner, J. R. Myosin light chain kinase: pulling the strings of epithelial tight junction function. Ann. N. Y. Acad. Sci. 1258, 34-42 (2012).

32. Butler, J. P., Tolić-Nørrelykke, I. M., Fabry, B. \& Fredberg, J. J. Traction fields, moments, and strain energy that cells exert on their surroundings. Am. J. Physiol. Physiol. 282, C595-C605 (2002).

33. Yissachar, N. et al. An Intestinal Organ Culture System Uncovers a Role for the Nervous System in Microbe-Immune Crosstalk. Cell 168, 1135-1148.e12 (2017). 
34. Wirtz, S. et al. Chemically induced mouse models of acute and chronic intestinal inflammation. Nat. Protoc. 12, 1295-1309 (2017).

35. Farquhar, M. G. \& Palade, G. E. Junctional complexes in various epithelia. J. Cell Biol. 17, 375412 (1963).

36. Zihni, C., Mills, C., Matter, K. \& Balda, M. S. Tight junctions: From simple barriers to multifunctional molecular gates. Nature Reviews Molecular Cell Biology 17, 564-580 (2016).

37. Harris, T. J. C. \& Tepass, U. Adherens junctions: From molecules to morphogenesis. Nature Reviews Molecular Cell Biology 11, 502-514 (2010).

38. Hartsock, A. \& Nelson, W. J. Adherens and tight junctions: Structure, function and connections to the actin cytoskeleton. Biochimica et Biophysica Acta - Biomembranes 1778, 660-669 (2008).

39. Magalhaes, J. G., Tattoli, I. \& Girardin, S. E. The intestinal epithelial barrier: How to distinguish between the microbial flora and pathogens. Seminars in Immunology 19, 106-115 (2007).

40. Tran, C. D. et al. Gut permeability, its interaction with gut microflora and effects on metabolic health are mediated by the lymphatics system, liver and bile acid. Future Microbiology 10, 13391353 (2015).

41. Kundu, P., Blacher, E., Elinav, E. \& Pettersson, S. Our Gut Microbiome: The Evolving Inner Self. Cell 171, 1481-1493 (2017).

42. Lee, J. L. \& Streuli, C. H. Integrins and epithelial cell polarity. J. Cell Sci. 127, 3217-3225 (2014).

43. Bachir, A. I., Horwitz, A. R., Nelson, W. J. \& Bianchini, J. M. Actin-Based Adhesion Modules Mediate Cell Interactions with the Extracellular Matrix and Neighboring Cells. Cold Spring Harb. Perspect. Biol. 9, a023234 (2017).

44. Su, L. et al. Targeted Epithelial Tight Junction Dysfunction Causes Immune Activation and Contributes to Development of Experimental Colitis. Gastroenterology 136, 551-563 (2009).

45. Clayburgh, D. R. et al. Epithelial myosin light chain kinase-dependent barrier dysfunction mediates T cell activation-induced diarrhea in vivo. J. Clin. Invest. 115, 2702-2715 (2005).

46. Salim, S. Y. \& Söderholm, J. D. Importance of disrupted intestinal barrier in inflammatory bowel diseases. Inflammatory Bowel Diseases 17, 362-381 (2011).

47. Martin, A. \& Devkota, S. Hold the Door: Role of the Gut Barrier in Diabetes. Cell Metabolism 27, 949-951 (2018).

48. Kelly, J. R. et al. Breaking down the barriers: the gut microbiome, intestinal permeability and stress-related psychiatric disorders. Front. Cell. Neurosci. 9, 392 (2015). 
Tight junctions

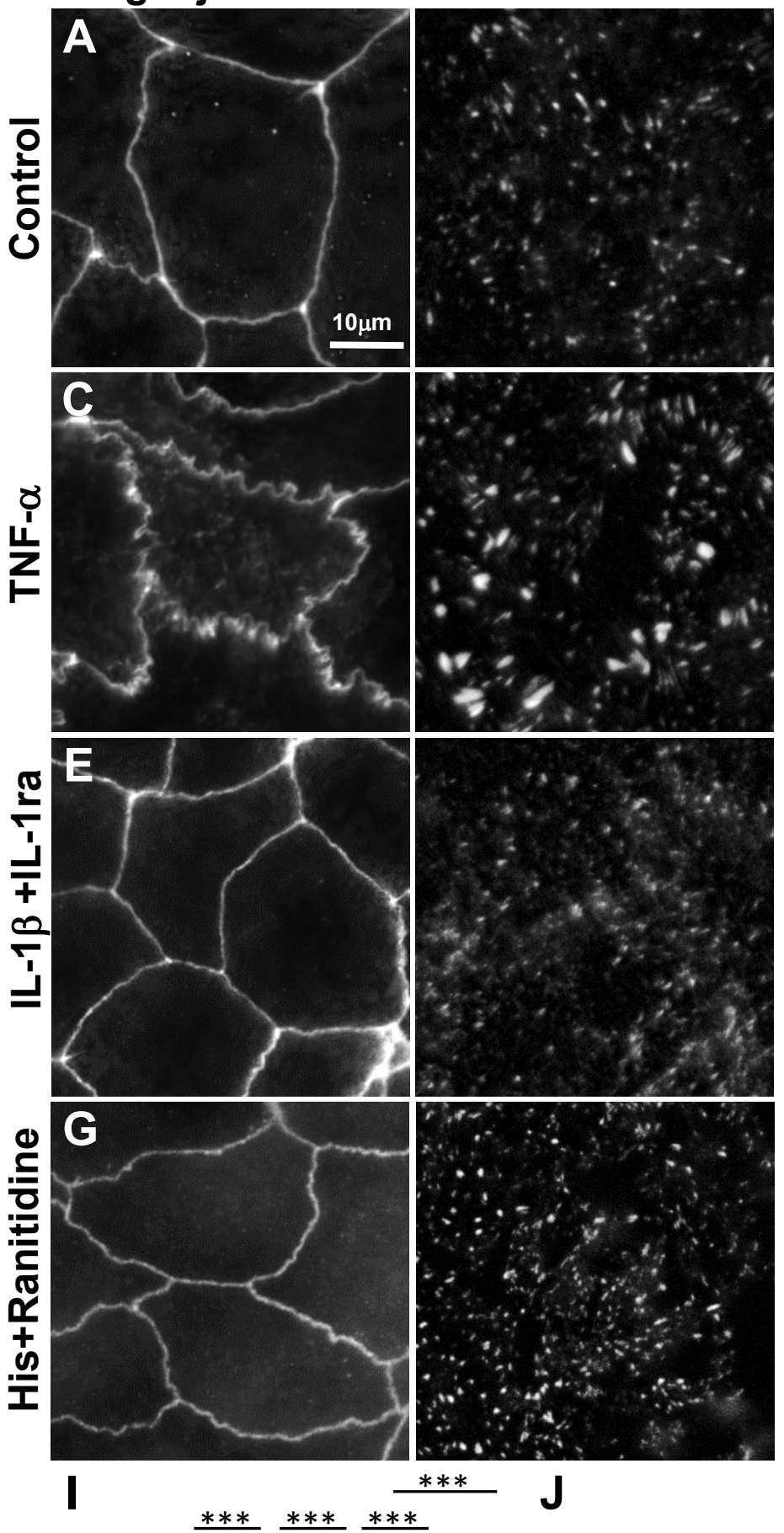

Tight junctions Focal adhesions
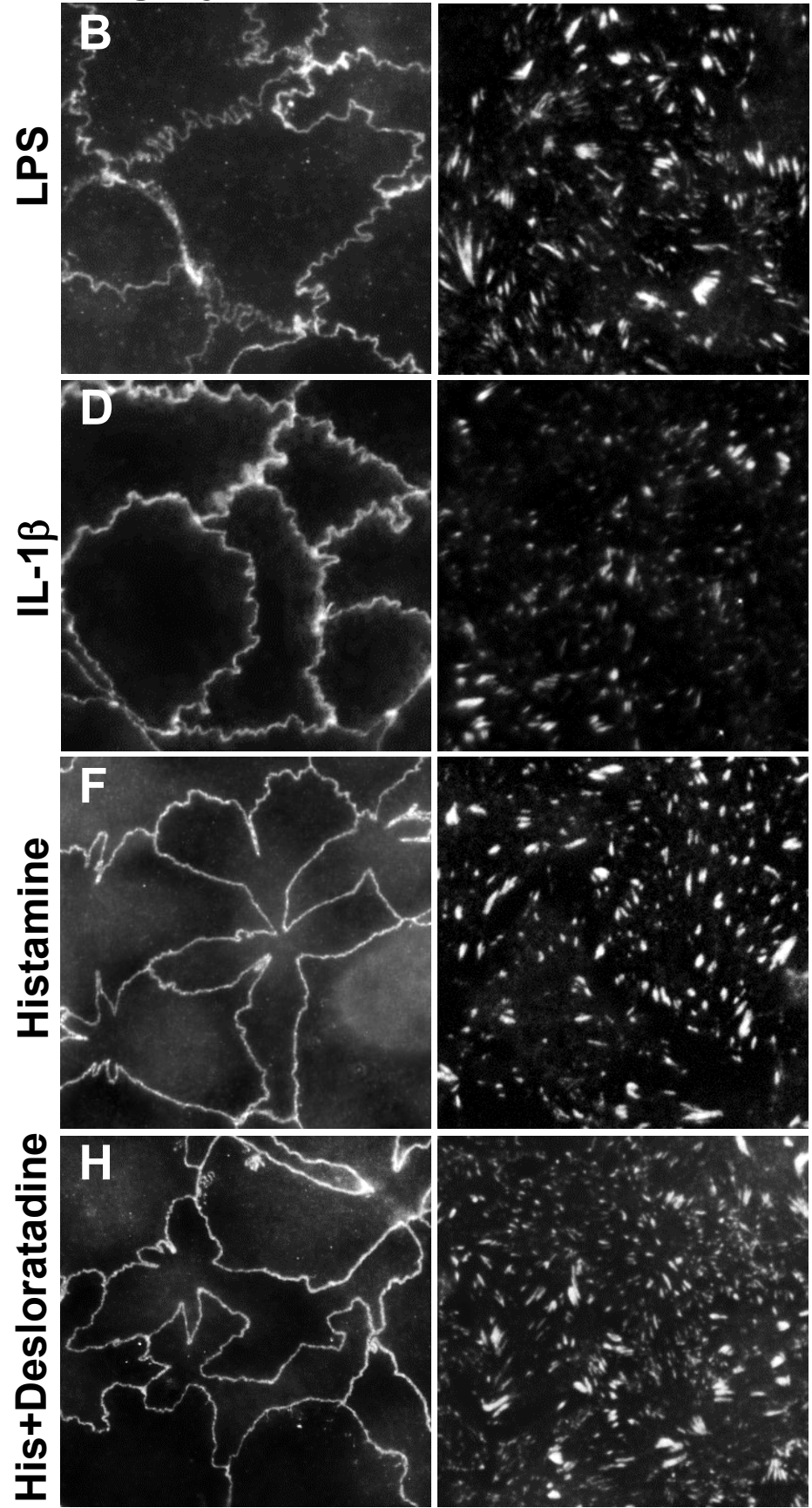

K

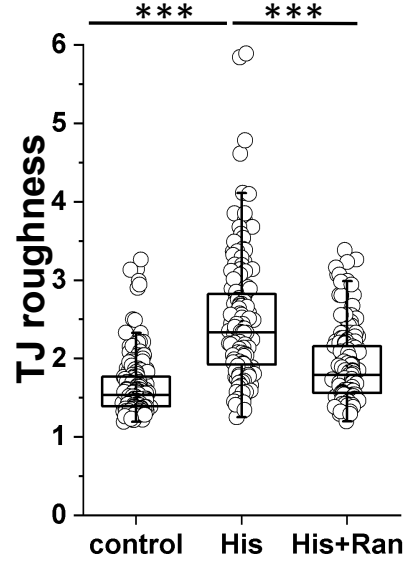

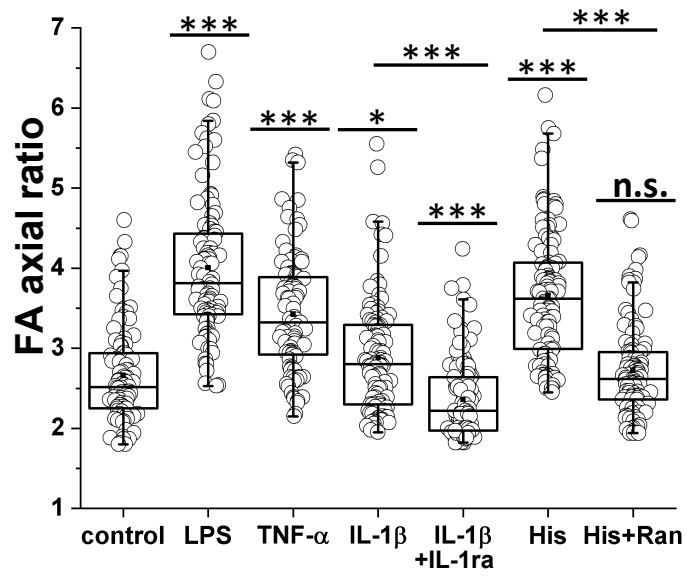



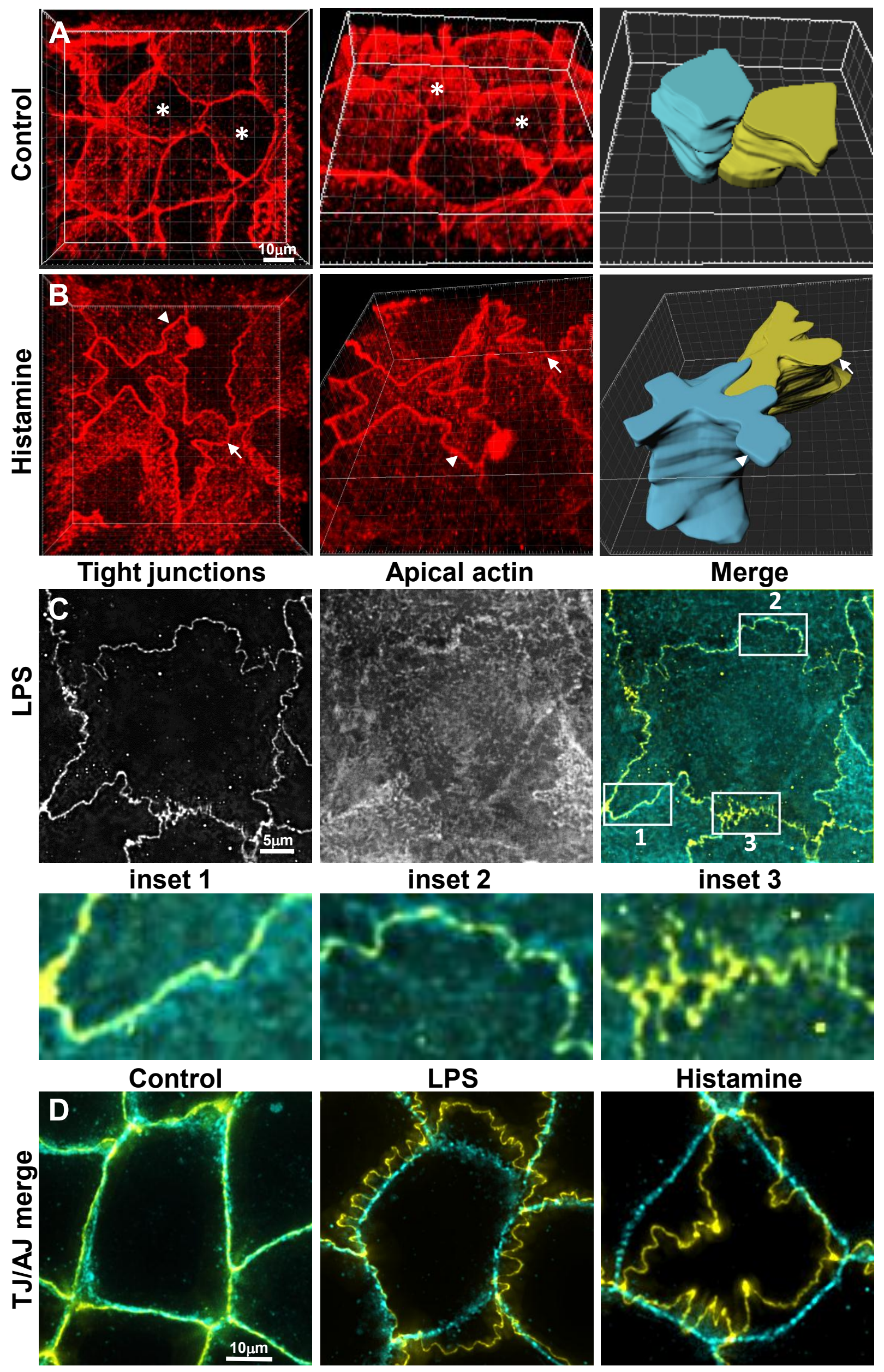

Figure 2 


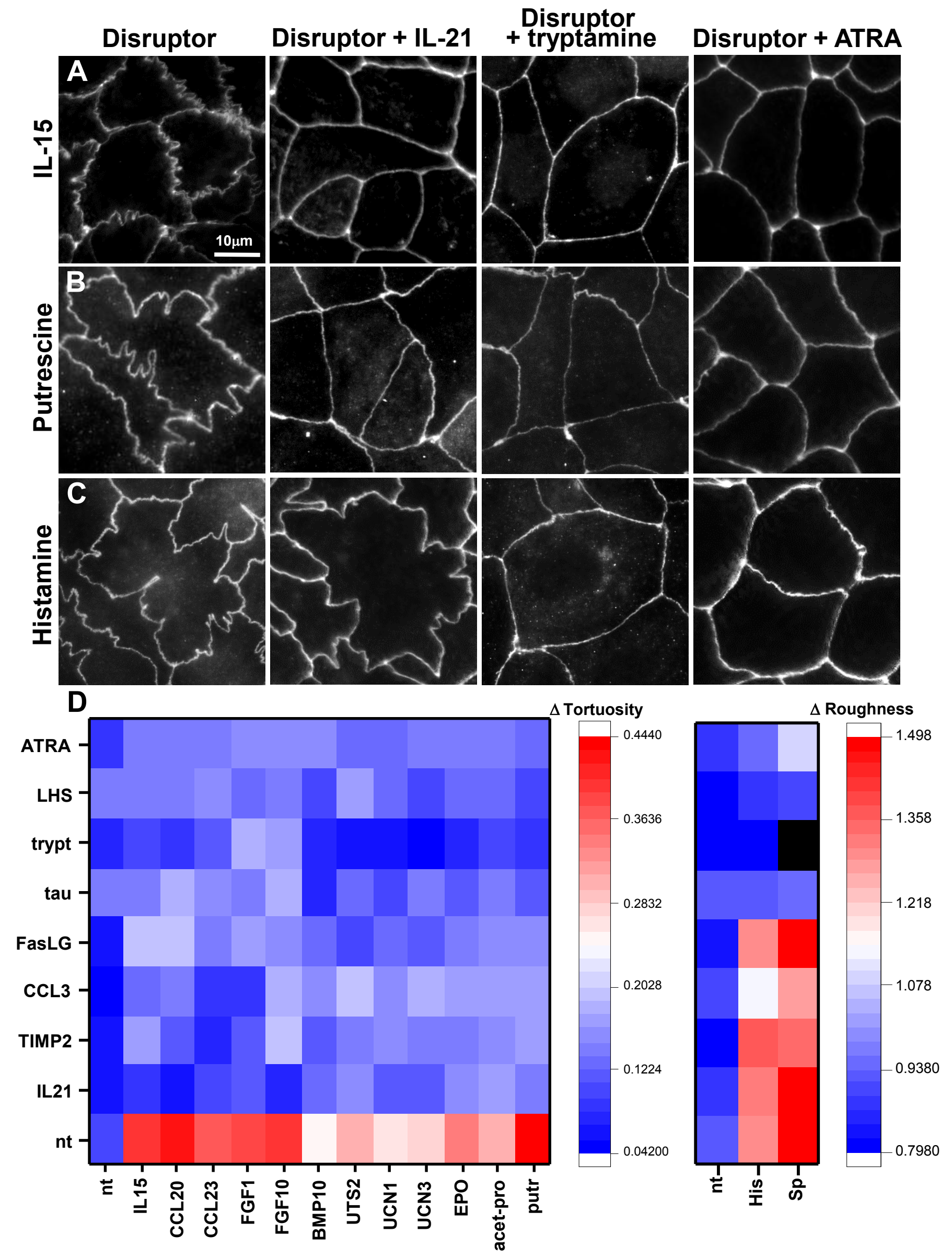

Figure 3 

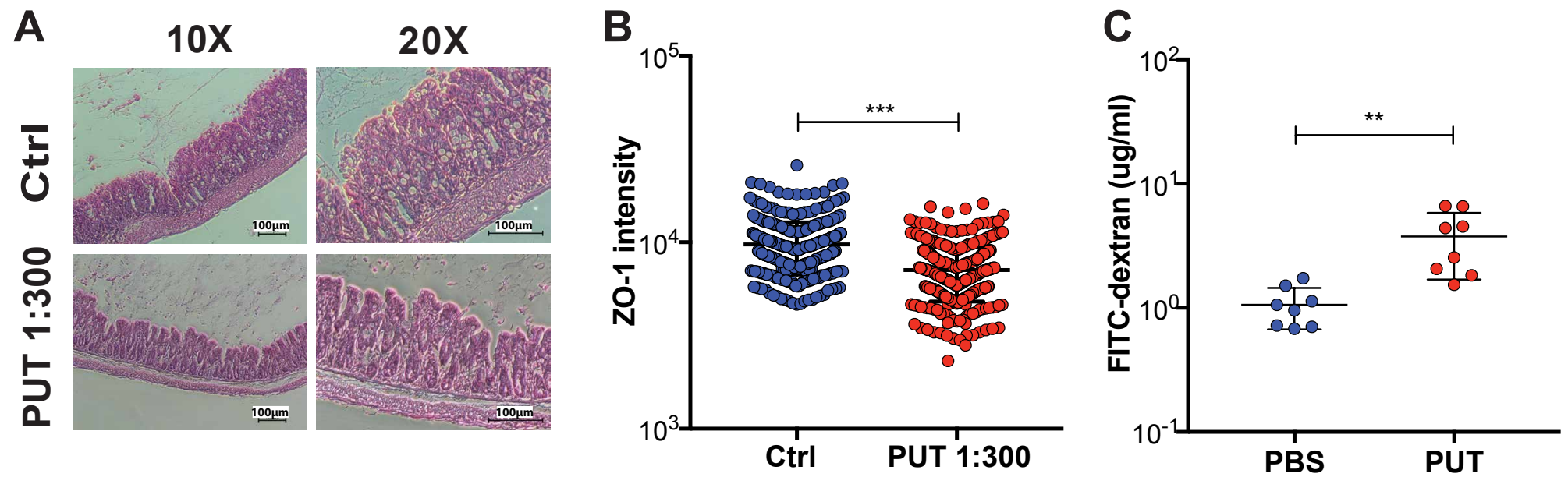

D

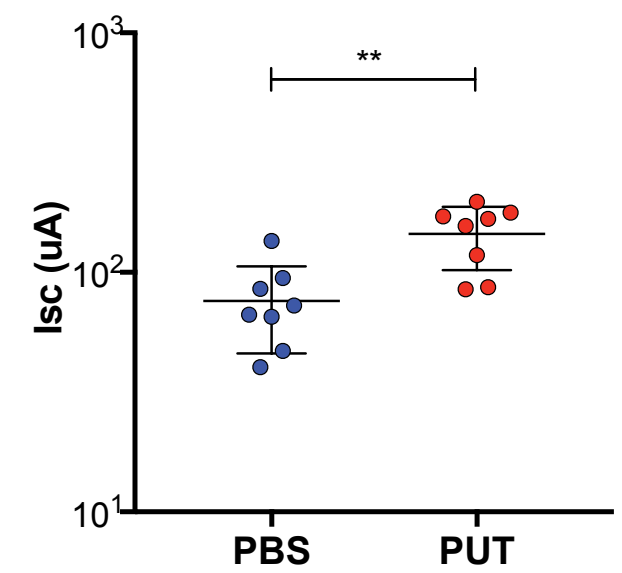

G

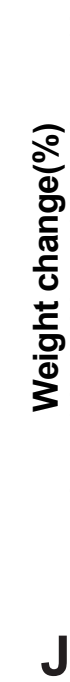

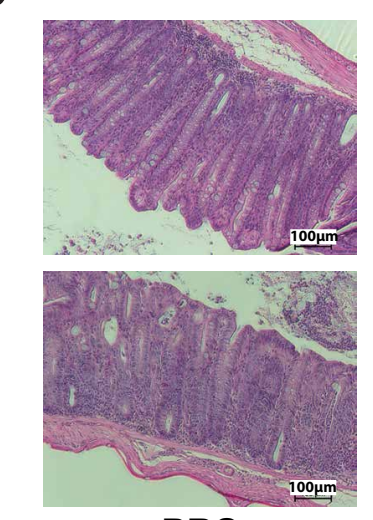

PBS
E

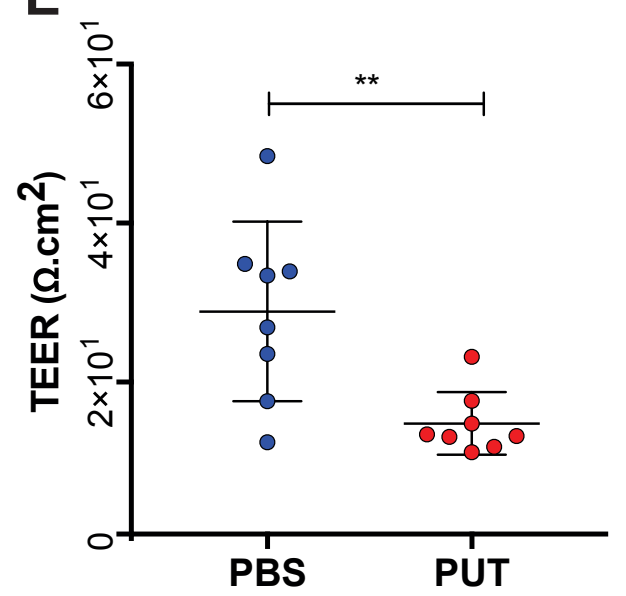

H

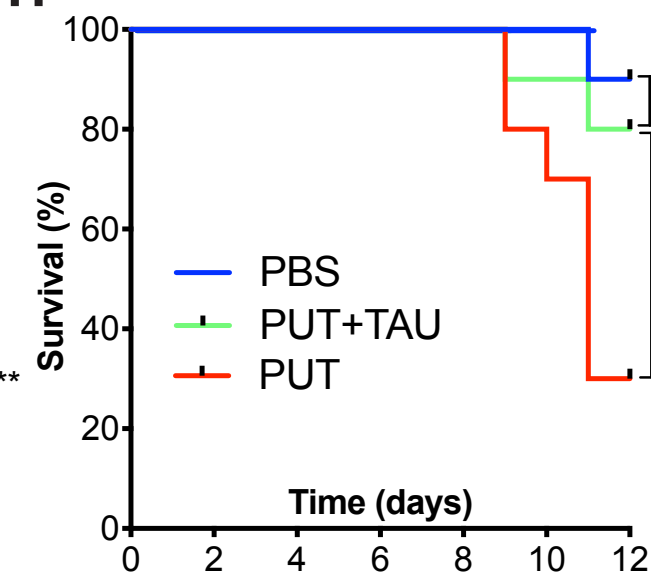

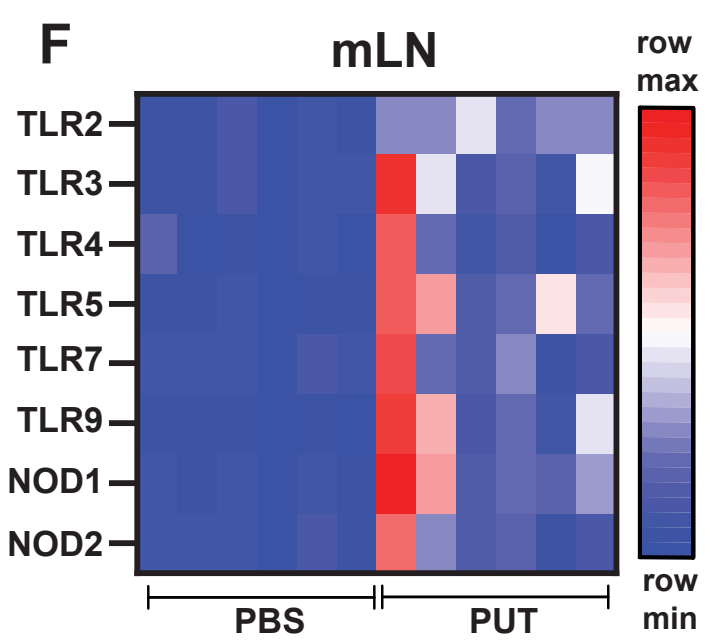

I

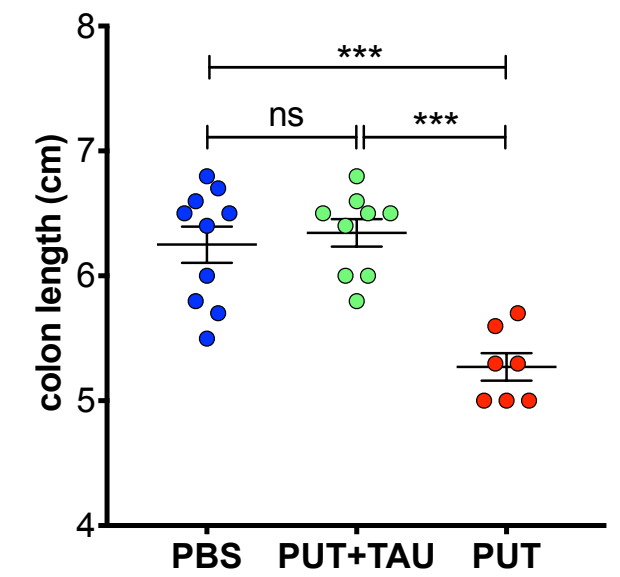

Figure 6

K

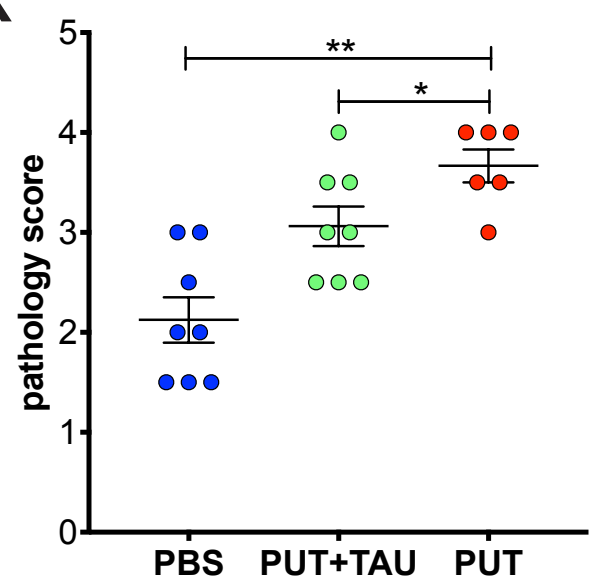



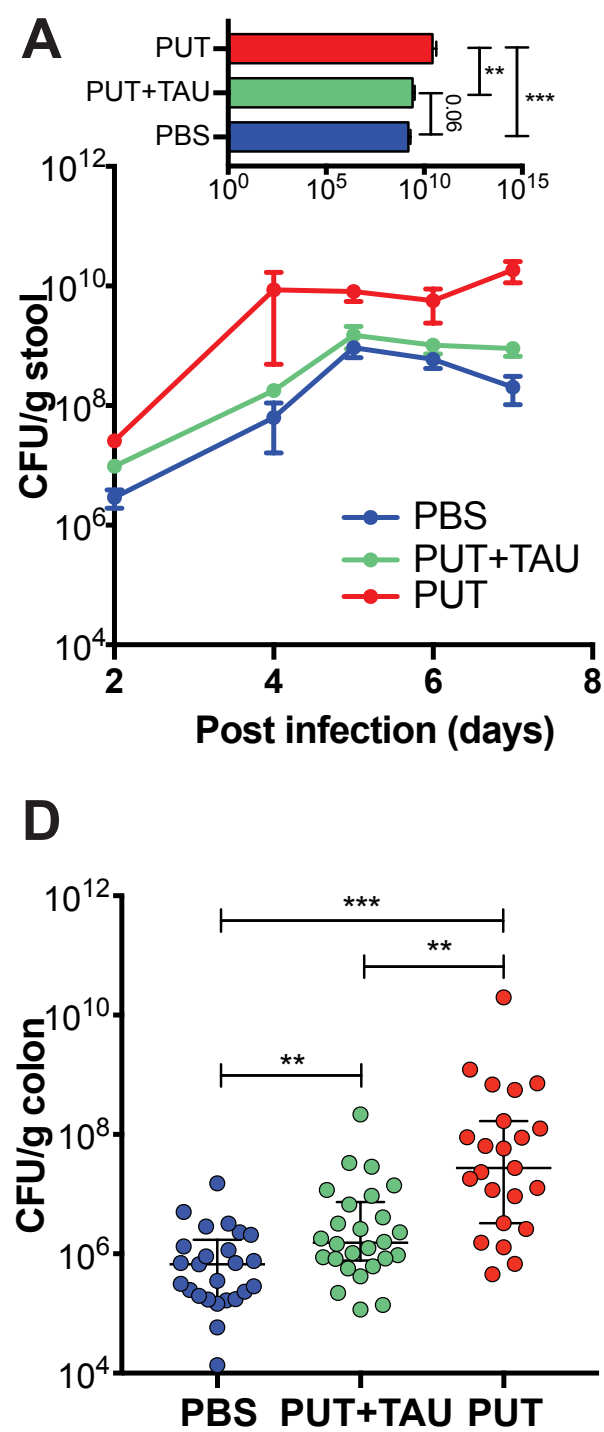

G

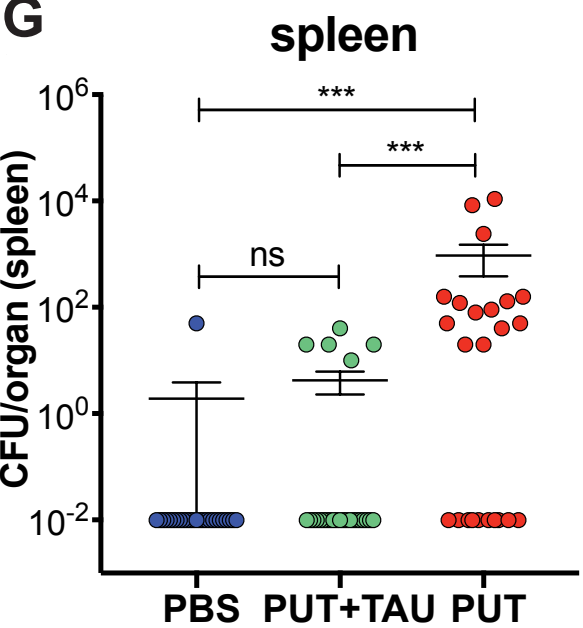

J

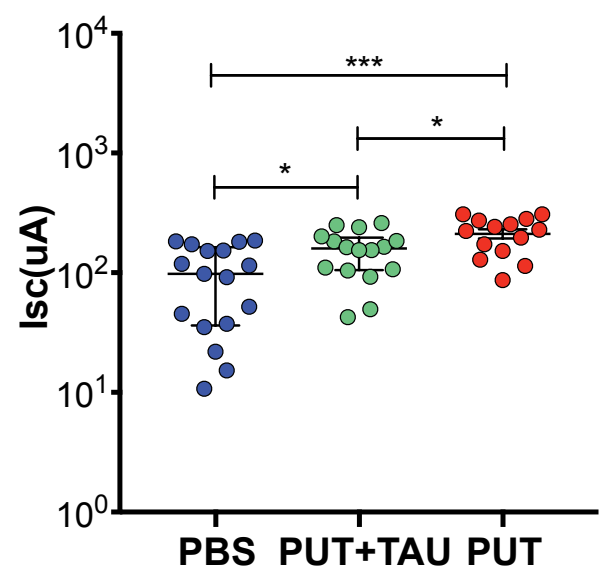

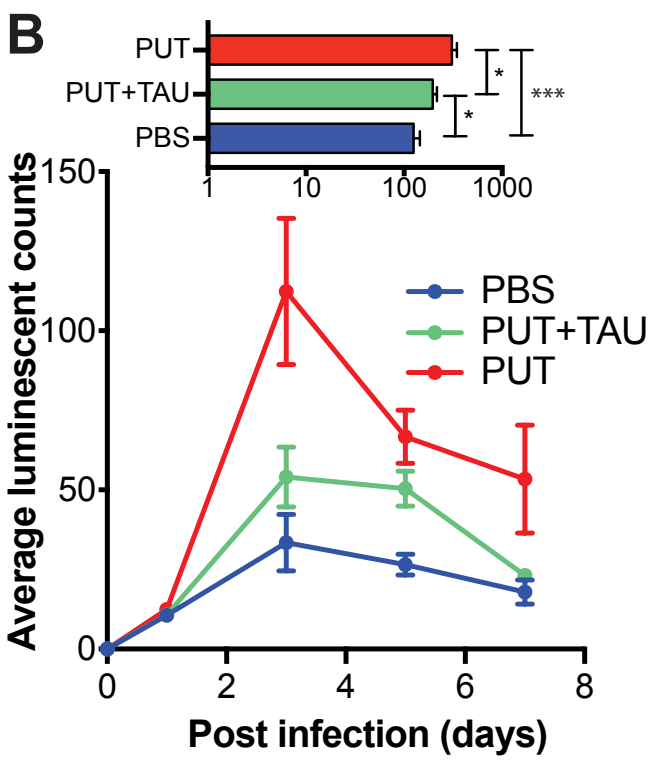

C
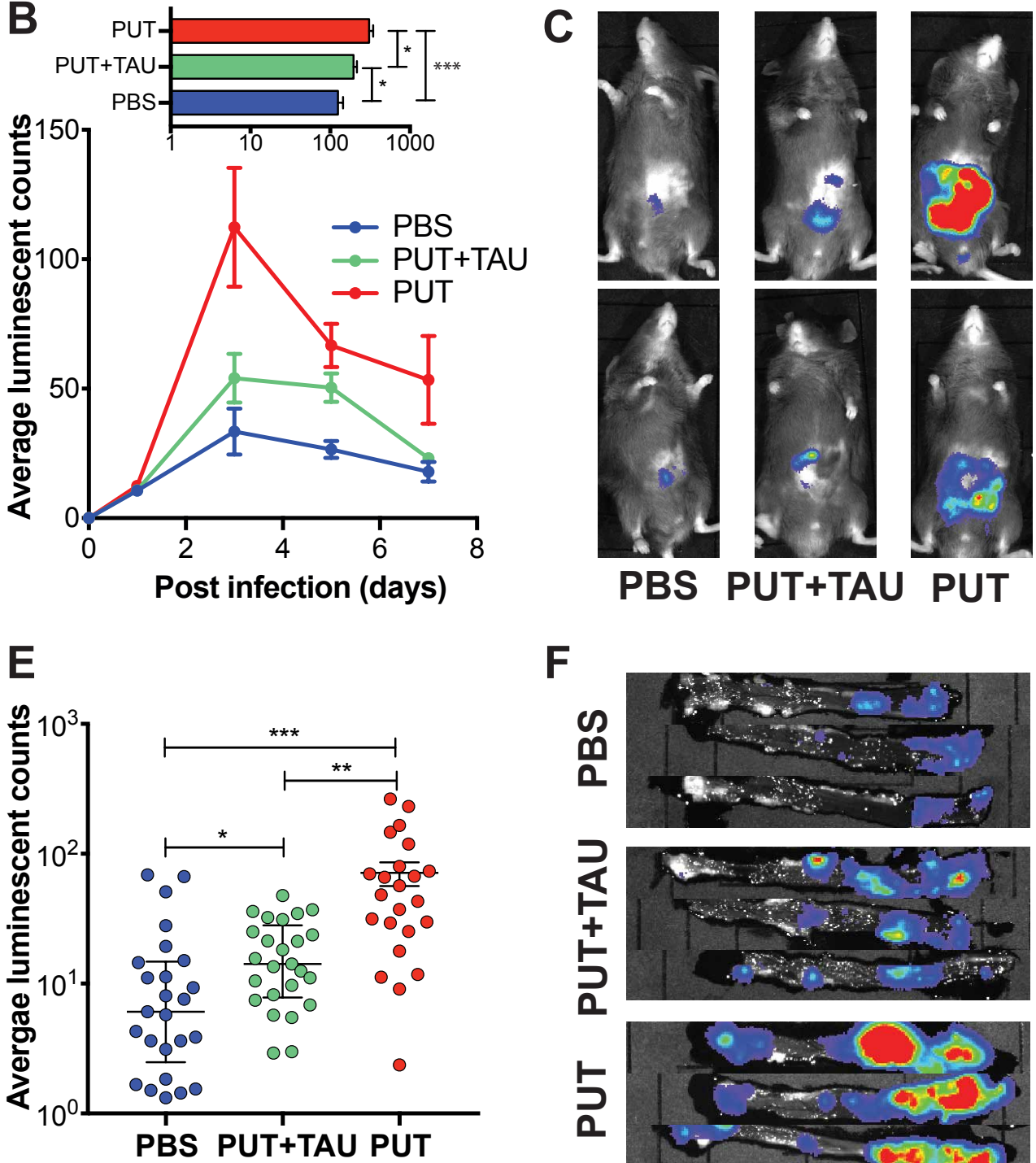

F

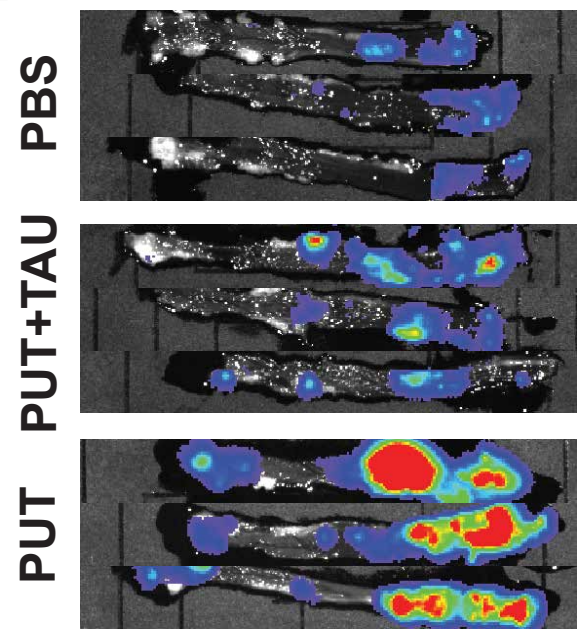

H

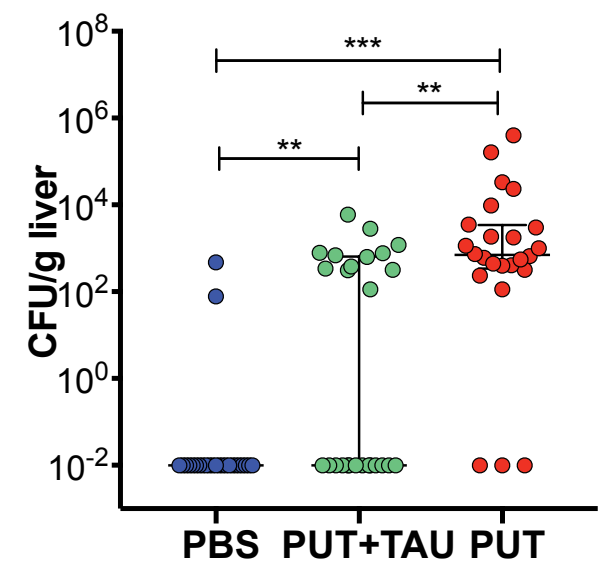

K

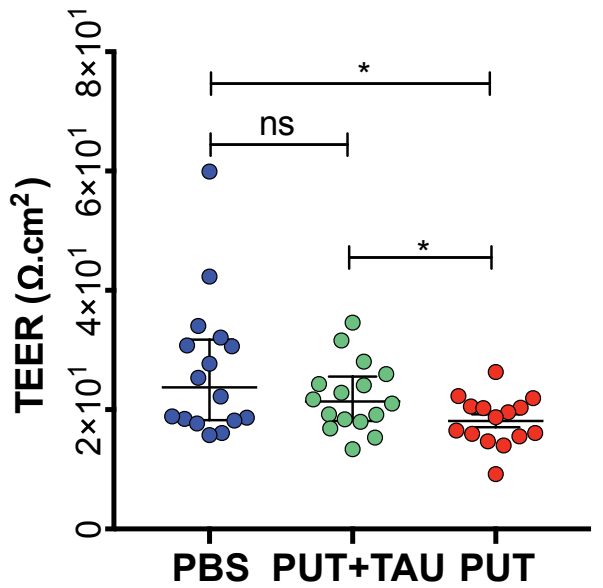

mLN

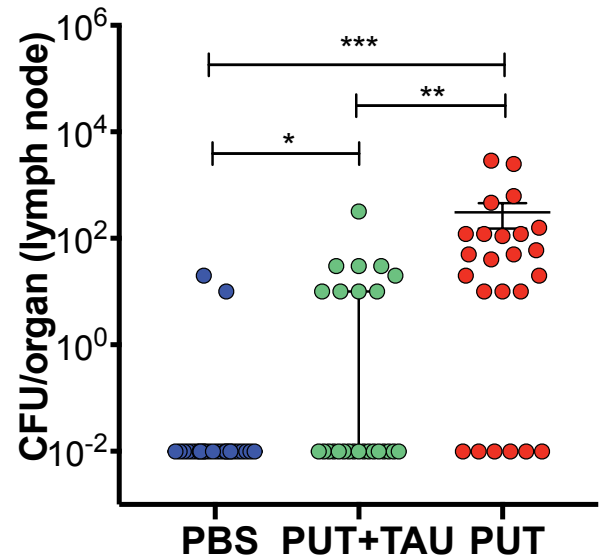

Figure 7 


\section{Supplemental Methods}

Cell substrate preparation: Collagen gels were prepared from $3 \mathrm{mg} / \mathrm{ml}$ rat tail collagen solution (Roche) via cross-linking with 1-ethyl-3-(3-dimethylaminopropyl) carbodiimide (EDC) and $\mathrm{N}$ hydroxysuccinimide (NHS) as previously described ${ }^{1} 20 \mu$ l of collagen/EDC/NHS mixture were applied to the bottom of each well in glass bottom 96 well plate, allowed to polymerize overnight at room temperature, equilibrated with cell culture medium for 2 hours at $37^{\circ} \mathrm{C}$ before cell plating. For fibronectin coating, glass bottom multi-well plates were coated with $50 \mu$ of bovine fibronectin (Biological Industries) solution $25 \mu \mathrm{g} / \mathrm{ml}$ in PBS for 1 hour at $37^{\circ} \mathrm{C}$ and rinsed 3 times with PBS before cell plating.

Cell labeling: For immunofluorescent staining cells were permeabilized and simultaneously fixed with 0.5\% Triton X100 in 3\% paraformaldehyde for 3 minutes and further fixed with 3\% paraformaldehyde for additional 30 minutes. TJ labeling was performed with either rabbit anti-cingulin antibodies (kindly provided by S. Citi, University of Geneva) or with anti-ZO1 mouse monoclonal antibodies (BD Transduction Laboratories). FAs were labeled with either anti-paxilin mouse monoclonal antibodies (BD Transduction Laboratories) or with rabbit polyclonal anti-zyxin antibodies (prepared in B. Geiger's lab). AJs were labeled with anti- $\beta$-catenin polyclonal rabbit antibodies (Sigma). The following secondary fluorescently labeled antibodies (Invitrogen) were used: Alexa Fluor 488 goat anti-mouse or anti-rabbit antibodies for tight junction visualization and Alexa Fluor 647 goat anti-mouse or antirabbit antibodies for FAs or AJs visualization. Actin was labeled with TRITC-phalloidin (Sigma) and nuclei with DAPI.

Morphometric image analysis: Cell images were segmented using Morphological Segmentation MorphoLibj plug-in ${ }^{2}$. Cell shape was quantified by Roughness $\left(\frac{\text { Perimeter }^{2}}{4 \pi \text { Area }}\right)$ which is the ratio of the area of the equivalent-perimeter circle to the area of the cell. This value is one for perfect circle and larger otherwise. We further quantified the shape of individual cell-cell edges by separating the edges at three-cell junctions, and computing the tortuosity of each edge as the ratio between the edge' length to the Euclidean distance between its end-points. This value is one for straight line and larger otherwise. The quantification was implemented as $\mathrm{Fiji}^{3}$ macro and is available at https://github.com/WIS-MICC-CellObservatory/Intestinal Barrier Function"

\section{Design of screening experiments}

Screening of human secreted molecules library: Library of 256 secreted molecules of human origin was assembled by Dr. R. Strausmann 4 . The final concentration of molecules was chosen in order to exceed the ED50 reported in literature. Two biological repeats of the cell treatment were performed. After visual examination of TJ morphology following treatments were identified as modulators of TJ 
organization: TNF - 50ng/ml; IL-15 - 25ng/ml; CCL-20 - 50ng/ml; CCL-23 - 50ng/ml; FGF-1 - 25ng/ml; FGF-10 - 100ng/ml; BMP-10 - 50ng/ml; UTS-2 - 10ng/ml; UCN-1 - 20ng/ml; EPO - 10ng/ml; UCN-3 - 20ng/ml; IL-21 - 30ng/ml; CCL-3 - 40ng/ml; TIMP-2 - 100ng/ml; FasLG - 10ng/ml (See also Supplemental Table 1).

Screening of bacterial metabolites library: Metabolomic analysis of small molecules presented in fecal extracts obtained from healthy wild-type (WT) and dysbiotic Asc-/- mice was previously described $^{5}$. From the list of metabolites increasingly abundant in dysbiotic conditions selected subset was selected for screening for their ability to cause TJ alterations (Supplemental Table 2).

From the list of compounds enriched in healthy intestine selected subset screened for their capacity to prevent tight junctions damage induced by co-treatment with $300 \mathrm{ng} / \mathrm{ml}$ LPS (Supplemental Table 2). Both screenings were performed at 1,5 and $10 \mathrm{mM}$ concentrations for all metabolites.

Robotic experimental design for high throughput screening of pharmacologically-active compounds: 2956 biologically active compounds (SelleckChem) at 10 $\mathrm{M}$ concentration were tested for their ability to disrupt or stabilize TJ organization. In order to create disease-mimicking conditions cells were treated with culture medium containing $300 \mathrm{ng} / \mathrm{ml}$ Lipopolysaccharide (LPS) and 10mM Histamine, in combination with the library compounds. Cell plating, drug treatment, fixation and labeling procedures in high throughput format were performed using the following equipment: Multidrop Combi Reagent Dispenser (Thermo Fisher Scientific) was used for fibronectin coating, cell dispensing, primary and secondary labeling mixture dispensing. Bravo Automated Liquid Handling Platform (Agilent) was used for addition of drug-containing medium, permeabilization with PFA/Triton and PFA fixation. EL406 Washer Dispenser (BioTek) was used for liquid aspiration and washes between fixation and labeling steps.

Traction force microscopy: SoftTrac 6 well plates with pre-fabricated collagen coated 12kPa hydrogels, containing embedded $0.2 \mu \mathrm{m}$ fluorescent beads, were purchased from Matrigen. Cells (200,000 per well) were plated and allowed to grow for 24 hours to form epithelial islands, treated with indicated compounds for another 24 hours and live imaged using DeltaVision microscope equipped with an environmental chamber. After acquisition of cell and fluorescent beads images in steady state conditions cells were detached with trypsin and imaging of beads was repeated in relaxed state. Displacement maps generation and force calculations were performed using MatLab script as previously described ${ }^{6}$.

Trans-epithelial electrical Resistance (TEER) and dextran permeability measurements: Cells were grown on polycarbonate Trans-well ${ }^{\circledR}$ membranes with $0.4 \mathrm{~mm}$ pores (Corning) for 7 days, indicated compounds were added inner and outer chambers for last 24 hours. TEER measurements were 
performed using EVOM2 Epithelial Voltohmmeter, equipped with STX2 electrode (World Precision Instruments) according to manufacturer's instructions. For dextran permeability test regular culture medium was replaced with phenol red free DMEM in outer chamber and same medium with $1 \mathrm{mg} / \mathrm{ml}$ of 70kD Rhodamine dextran (Sigma) was added into inner chamber for 2 hours. Medium from the outer chamber was collected and Rhodamine fluorescence was measured using Infinite M200 Pro Microplate reader (Tecan).

Mice: Animals were maintained and studies were conducted according to the Institutional Animal Care and Use Committee Standard Operating Procedure (IACUC SOPs). All experimental procedures were approved by the local Institutional Animal Care and Use Committee 20120815-2. Wild-type (WT) C57BL/6 male mice were purchased from Envigo and acclimatized to the animal facility environment for 2 weeks before experiments. All mice were kept under strict 12-h light-dark cycle (lights on at 6 am off at $6 \mathrm{pm})$. Mice were 8 to 9 weeks of age when used for all in vivo experiments. In ex vivo colon organ culture experiment, 2-week-old SPF WT C57BL/6 mice were used.

For putrescine treatment, mice received putrescine by oral gavage with a dose of $200 \mathrm{mg} / \mathrm{kg}$ twice daily for 10 days prior to and during DSS treatment or Citrobacter rodentium infection. For taurine treatment, mice received $30 \mathrm{mg} / \mathrm{ml}$ taurine in their drinking water for 10 days prior to and during infection models.

Ex vivo intestinal organ culture system: We used a three-dimensional device for gut organ culture and experiment, which was previously described in detail ${ }^{7}$. Briefly, intact whole colons from 14-dayold SPF mice were dissected sterilely. The solid contents in the colon lumen were flushed gently. The colon fragment was then threaded with a sterile surgical thread and fixed across the luminal input and output ports. The gut culture device was maintained at $37^{\circ} \mathrm{C}$ on a controlled heating block, and colon tissues were half-soaked in sterile medium at a constant flow. The tissue culture medium, containing different concentrations of putrescine, was loaded into a $10 \mathrm{ml}$ syringe and continuously infused into the device input ports by a syringe pump (flow rate of $1 \mathrm{ml} / \mathrm{h}$ ). Medical degrade $5 \% \mathrm{CO}_{2}$ and $95 \% \mathrm{O}_{2}$ gas mixture is provided to the device from a compressed gas cylinder connected to a regulator. After culturing for 2 hours, colon tissues were harvested, fixed in 4\% paraformaldehyde, paraffin-embedded and sectioned for HE staining and immunofluorescence staining.

DSS-induced colitis: The animals were weighed daily, monitored for signs of distress and rectal bleeding. Colonoscopy was performed on day 7 using a high-resolution mouse video endoscopic system (Carl Storz, Tuttlingen, Germany). The severity of colitis was scored blindly using MEICS (Murine Endoscopic Index of Colitis Severity), which consists of five parameters: granularity of mucosal surface; vascular pattern; translucency of the colon mucosa; visible fibrin; and stool consistency ${ }^{8}$. Mice were sacrificed for measurement of colon length and histological analysis on day 
12. Pathological severity was scored by a pathologist in a blind manner, based on tissue damage and infiltration of inflammatory cells ${ }^{9}$.

Citrobacter rodentium infection: Mice were infected with $200 \mu$ l solution containing approximately 1 $\times 10^{9}$ colony-forming units (CFU) of overnight cultured bacteria by oral gavage. Infection was monitored by enumerating stool CFU and measuring abdominal bioluminescent. For in vivo bioluminescent imaging, mice were anesthetized and bioluminescence was measured using an IVIS2000 instrument and Living Image software (Perkin Elmer). For ex vivo luminescence quantification, the whole colons were dissected, extensively washed from all luminal contents, longitudinally cut open and immediately imaged. For CFU counts, stool or tissues were collected, weighed and homogenized in sterile phosphate buffered saline (PBS-/-). Homogenates were then serially diluted in PBS-/- and plated on LB kanamycin plates, incubated overnight at $37^{\circ} \mathrm{C}$. Bacterial CFUs were counted the next day, normalizing them to the stool or tissue weight.

Measuring intestinal permeability by FITC-dextran: On the day of FITC-dextran assay, 4 kDa fluorescein isothiocyanate (FITC)-dextran (Sigma, FD4) was dissolved in PBS-/- to a concentration of $80 \mathrm{mg} / \mathrm{ml}$. Mice were fasted for 4 hours prior to gavage with $200 \mu \mathrm{l}$ dextran. Mice were anesthetized 3 hours following gavage and blood was collected and centrifuged at $10,000 \times \mathrm{g}$ for 12 minutes at $4^{\circ} \mathrm{C}$. Serum was collected and fluorescence was quantified at an excitation wavelength of $485 \mathrm{~nm}$ and an emission wavelength of $535 \mathrm{~nm}$.

Ussing chamber: An Ussing chamber system (Warner Instruments P2300) was used to measure transport across colon epithelial membranes according to the manufacturer's instructions. Briefly, after calibration of EasyMount chambers, colonic tissue was dissected from mice, gently washed, cut open and immediately mounted on the EasyMount Inserts. Voltage clamp modes were performed and short-circuit current as well as trans-epithelial electrical resistance were recorded. Colon tissues were kept at $37^{\circ} \mathrm{C}$ in the chambers in physiological salt solution throughout the duration of the recording.

Quantification of microbial products in organs: We used a series of pattern recognition receptor (PRR) reporter cell lines (InvivoGen, HEK-Blue TLR and NLR reporter cell lines) for quantification of microbial products, including TLR2, TLR3, TLR4, TLR5, TLR7, TLR9, NOD1, NOD2. Lymph node, spleen, and liver were harvested from mice, homogenized in PBS-/-, and added to reporter cell lines incubated with HEK-Blue Detection medium (InvivoGen) according to the manufacturer's instructions. Secreted embryonic alkaline phosphatase (SEAP) activity was assessed by reading the optical density at 620-655 $\mathrm{nm}$ with a microplate reader. 
Immunofluorescence staining: Colon samples were dissected, extensively washed, fixed in $4 \%$ paraformaldehyde, paraffin-embedded and sectioned. Sections were deparaffinized and subsequently antigen retrieved in $10 \mathrm{mM}$ citric acid, $\mathrm{pH}$ 6. Slides were blocked with PBS-/- containing $20 \%(\mathrm{v} / \mathrm{v})$ normal horse serum and $0.2 \%(\mathrm{v} / \mathrm{v})$ Triton X-100 for 1 hour, and then incubated with rabbit anti-ZO1 primary antibody (Invitrogen $40-2200$ ) at $4^{\circ} \mathrm{C}$ overnight. Sections were washed and incubated for 1 hour at room temperature with Alexa 488-conjugated donkey anti rabbit secondary antibody. Alternatively, sections were stained with Ki67 to assess proliferating cells or anti-cleaved caspase 3 antibody to indicate apoptotic cells.

Flow cytometry: Colons were dissected from mice, extensively washed from fecal matter followed by $2 \mathrm{mM}$ EDTA dissociation in $37^{\circ} \mathrm{C}$ for $20 \mathrm{~min}$. Following extensive shaking, the epithelial fraction was discarded and colons were then digested using DNAase I and Collagenase for lamina propria analysis. The isolated cells were washed with cold PBS and resuspended in PBS containing 1\% BSA. For cell surface staining, single-cell suspensions were blocked and stained with antibodies against CD45, CD3, CD4 and RORgt for 30 minutes on ice. For cytokine staining, cells were first incubated in Iscove's Modified Dulbecco's Medium (IMDM) for 4 hours at $37^{\circ} \mathrm{C}$ and then stained with antibodies against IL17A, IFN- $\gamma$, IL-22 for 30 minutes on ice. Stained cells were analyzed on a BD-LSR Fortessa cytometer and were analyzed with FlowJo software.

Intestinal epithelial cell sorting, RNA isolation and RNA-sequencing: Colonic epithelial cells form mice were dissociated in HBSS containing $2 \mathrm{mM}$ EDTA, collected, filtered and stained with anti-EpCAM and anti-CD45 antibodies (Biolegend) for 30 mins on ice. Cells were then sorted into RLT buffer (Qiagen, 79216) supplement with 40mM DTT. The cell solution was cleaned with 2X AM pure beads (Beckman Coulter), and reverse transcription was performed directly on the cells with the beads. Samples after reverse transcription were pooled together, and cDNA amplification was performed to construct Nextera XT DNA libraries (Illumina, FC-131-1024) following manufacturer's instructions. Libraries that passed quality control were loaded with a concentration of $2 \mathrm{pM}$ on 75 cycle high output flow cells (Illumina, 20024906) and sequenced on a NextSeq 500 (Illumina). RNA-sequencing reads were aligned to the mouse reference Gencode M24 using STAR algorithm (version 2.7.3a). Differential gene expression analyses between groups were performed by using DESeq2 method (version 1.28.0). Gene ontology enrichment analysis was performed using the R package ClusterProfiler.

Data integrity check: Figures and supplemental figures were checked for data integrity using the Proofig pipeline.

Statistical Analysis: Data were expressed as mean \pm SEM. P values $<0.05$ were considered significant $(* \mathrm{p}<0.05 ; * * \mathrm{p}<0.01 ; * * * \mathrm{p}<0.001)$. Pairwise comparisons were performed using Student's $\mathrm{t}$ test. Mann-Whitney $U$ test was used when data was not normally distributed. 


\section{Supplemental Results}

\section{Development of an in vitro system for monitoring TJ and FA integrity}

The inner lining of the intestine is comprised of a single layer of columnar epithelial cells. These cells are attached, via their basal aspect to the underlying basement membrane, while at their apical aspect they are attached to neighboring cells via TJs and adherens junctions, which seal and mechanically reinforce the intercellular contact. To morphologically quantify the bio-physical character and integrity of this important facet of intestinal barrier function, we developed a microscopy-based system allowing the high-throughput in vitro characterization and quantification of TJ and FA integrity by using cultured human intestinal CaCo-2 cells. These cells display multiple differential markers characteristic for normal colonic epithelial cells as well as the ability to form polarized monolayer with well-organized apical TJs ${ }^{10,11,12}$. The apical and basal adhesions were visualized by immunofluorescent labeling of their characteristic protein components, zonula occludens-1 (ZO-1) and zyxin, respectively (Supplemental Figure 1). TJs were visualized as a circumferential junctional belt by ZO-1 staining (Supplemental Figure 1A, B), while FAs appeared as dot- and dash-shaped structures by zyxin staining (Supplemental Figure 1A, C). TJs and FAs were localized to the apical and basal aspects of the cells, separated by at least $15 \mu \mathrm{m}$ from each other (Supplemental Figure 1A). Both TJs and FAs were associated with the F-actin- based cytoskeleton, as manifested by double labeling (Supplemental Figures 1B, C). Automated image analysis tools allowed us to identify individual TJs and FAs (Supplemental Figure 1D) and to quantify their morphometric parameters, which provided important readouts for effects of various external perturbations affecting epithelial barrier function as discussed below. Collectively, our in vitro screening system provides a novel, direct and intuitive approach to visualize and assess the cell junctional integrity in combination with other cytoskeletal structures.

Screening of a microbiota-associated metabolite library. All candidate molecules were screened under two conditions. First, individual metabolites were cultivated with CaCo-2 cells, in search for Type-I or II intestinal barrier function disruptive phenotypes. In addition to histamine (Figure 1F) we identified three novel disruptive metabolites: acetyl-proline causing Type-I effect (Supplemental Figure 3A, B), spermine inducing Type-II disruption (Supplemental Figure 3C) and putrescine characterized by a mixed Type-I/II phenotype (Supplemental Figure 3D). In addition to disruptive effect on TJs, newly identified metabolites also induced enlargement of FAs (Supplemental Figure 3A-D). Second, to search for potential TJ stabilizers, we treated cells with individual candidate metabolites in the presence of LPS (300ng/ml). Using this approach, 3 stabilizing metabolites: taurine, tryptamine and L-homoserine, were uncovered to block LPS-induced disruption and FA enlargement (Supplemental Figure 3E-H). 
Screening of the library of pharmacologically bioactive compounds. We expanded the screening approach to a high-throughput scale, using fully automated experimental protocol which enabled us to screen large numbers of compounds at a single run, and automatically and systemically quantify their effects. To this aim, we selected a library of 2956 biologically active compounds provided by Selleck Chemicals LLC $\mathrm{Co}^{13}$. This small molecule library contains FDA/EDA-approved drugs, compounds that reached preclinical and clinical research stages, specific inhibitors of multiple signaling pathways, and compounds of natural origin. To search for novel epithelial stabilizers, we preformed the screening under moderately destabilized, 'disease-mimicking' conditions, achieved by co-treatment of the cells with low levels of LPS and histamine. This treatment caused a combined "Type-I" and "Type-II" phenotype, which allowed us to identify new molecules that either enhance or prevent the morphological changes. Using this approach, we identified 51 molecules possessing a stabilization capacity (selected examples shown in Supplemental Figure 4A), as well as 31 compounds that further enhanced TJ disruption upon destabilizing treatment by LPS/histamine (Supplemental table 3). Disruptive effect of selected novel candidate disruptors was validated by treatment in the absence of the TJ-disrupting LPS/Histamine mixture (Supplemental Figure 4B). Labeling of FAs further reinforced the inverse correlation of compounds' effect on TJs and FAs, as featured by disrupted TJ but enhanced FA (Supplemental Figure 4A, 4B, bottom panels). Altogether, our high-throughput / high-content screening approach identified a broad range of novel modulators of intestinal barrier function, derived from host secreted, microbial or pharmacological origins.

Restoration of pre-existing disrupted phenotype by epithelial stabilizers. To determine whether stabilizers can also restore TJ integrity in epithelial cells already displaying barrier distortion (rather than just blocking their formation), we performed sequential disruption-restoration treatments, namely, a 24-hour treatment with a disruptor alone, followed by another 24 hours with either a stabilizer or regular culture medium or a stabilizer-disruptor mixture. Specifically, CaCo-2 cell were treated with different disruptors (LPS, histamine, putrescine) for 24 hours, and then treated with different stabilizers (taurine or tryptamine) for additional period of 24 hours, in the presence or absence of the original disruptor, and then fixed and tested for TJ integrity. The results indicated that TJ disruption caused by LPS or histamine could be reversed by either washing the disruptors out or by the late addition of stabilizing metabolites (taurine or tryptamine) to the disruptor-containing medium (Supplemental Figure 5A, C). Disruption caused by putrescine was more severe than destabilizing treatment by LPS/histamine, manifested by more pronounced structural changes and slower recovery upon drug removal, yet the recovery of TJs after putrescine treatment was greatly improved by the addition of the stabilizers taurine or tryptamine (Supplemental Figure 5A, bottom panel, Supplemental Figure 5C).

Epithelial disruptors and stabilizers have similar morphological effect in different cultured cells of gastrointestinal origin. 
In order to validate the general relevance of our primary test system, which was based on utilization of cultured CaCo-2 cells, we applied small subset of newly-identified disruptors and stabilizers to a different cell line, T84, that similarly to CaCo-2 was derived from colorectal adenocarcinoma and proved to be ab excellent model for testing epithelial polarity and tight junction functionality. These cells show markers of colonocyte differentiation while CaCo-2 are considered to be resembling small intestinal enterocytes ${ }^{14}$. TJ in control T84 culture have regular polygonal shape, while treatment with putrescine, histamine or LPS causes significant distortion of TJ geometry, resembling type II (putrescine, histamine) or type I (LPS) disruption previously observed in CaCo-2 cells (Supplemental figure 6A). Co-treatment with stabilizing metabolite taurine or with stabilizing pharmacological agent - ATRA prevented disruptor-induced morphological changes of TJ in all cases (Supplemental figure $6 B, C)$.

Increase of acto-myosin contractility can cause TJ disruption, which can be prevented by treatment with TJ stabilizers.

We further tested the possibility that increase of cellular contractility, by itself, can be sufficient for altering TJ morphology. Towards this end, we treated cells with the pharmacological phosphatase inhibitor Calyculin A, which is a high potent PP1 phosphatase inhibitor. Calyculin A is widely used to increase cell contractility due to the inhibition of Myosin Light Chain Phosphatase (MLCP), which results in increased myosin phosphorylation, bringing it to an activated state ${ }^{17}$.

As shown, already within 30 minutes after Calyculin A addition at the concentration as low as $5 \mathrm{nM}$, signs of type I disruption could be detected in TJ morphology of treated cells, and 1 hour of incubation resulted in augmentation of type I disruption followed by transition to type II phenotype, seen after two hours of treatment (Supplemental figure 7A upper panel). Similar trend was observed upon incubation with 10nM Calyculin A, namely, initial appearance of type I disruption, more prominent than at $5 \mathrm{nM}$ concentration, with subsequent transition to type II morphology. Finally, the highest tested concentration of Calyculin A (20nM) caused pronounced type II disruption already after 30 minutes in the presence of drug (Supplemental figure 7A bottom panel). Such dose and time dependence of extent of epithelial disruption on contractility up-regulator suggests, that type II TJ morphology is actually an advanced state of epithelial disruption caused by significant increase in contractility, while type I morphology is caused by more moderate elevation of contractile forces. We would like to note that longer treatments with Calyculin A resulted in cell death in dose dependent manner, with 20nM concentration causing toxicity already in 2 hours.

Next we decided to examine whether disruptive effect of Calyculin A on TJ morphology can be prevented by treatment with epithelial stabilizers. All our previous experiments were performed following 24 hours incubation of the cells with disrupting or stabilizing agents. Since Calyculin A affects MLCK phosphorylation causing contractility increase in a relatively short time scale, and induces toxicity upon longer incubation, we employed two experimental approaches for combined treatment with stabilizing molecules. First cells were co-incubated for one hour with a mixture of 
Calyculin A and selected stabilizing molecule before fixation and labeling for TJ. Alternatively, cells were pre-incubated with selected stabilizers for 24 hours and then subjected for one-hour coincubation with mixture of Calyculin A and stabilizer. One-hour co-incubation with Calyculin A or ATRA turned out not to be sufficient for preventing Calyculin A induced disruption. However, cells preincubated with Taurine or ATRA for 24 hours didn't show epithelial disruption after additional onehour treatment with Calyculin A (Supplemental figure 7B upper panel). Interestingly, third examined stabilizer Tryptamine was capable of preventing disruption upon both experimental conditions; onehour co-treatment was sufficient to prevent TJ disruption caused by Calyculin A (Supplemental figure 7B bottom panel). Previous studies addressing role of acto-myosin contractility in regulation of TJ integrity reported that impairment of EBF upon treatment with inflammatory cytokines is caused by increase cellular contractility due to transcriptional up-regulation of MLCK leading to increased myosin light chain phosphorylation ${ }^{18}$. This observation allows us to propose that the stabilizing effect of taurine and ATRA against Calyculin A induced TJ disruption could also be achieved via transcriptional re-programming, requiring 24 hour of pretreatment to bring cells into protected state. Tryptamine, on the other hand, might have different mode of action acting through other contractility-reducing pathways with the kinetics similar to Calyculin A.

Disruptive effect of putrescine in mice with $\boldsymbol{C}$. rodentium infection. Putrescine treatment of mice infected with a bioluminescent variant of $C$. rodentium exacerbated disease, as noted by enhanced fecal shedding of viable C. rodentium (Supplemental Figure 10A) and higher bacterial burden, visualized by abdominal bioluminescence (Supplemental Figure 10B, C). Notably, mice treated with putrescine featured significantly enhanced bacterial attachment to the colonic epithelium, as indicated by greater burden of live bacteria in colon mucosa (Supplemental Figure 10D) and higher colonic bioluminescence following washing of intestinal content (Supplemental Figure 10E, F). Putrescine-treated mice also featured enhanced systemic $C$. rodentium colonization, including at the spleen, liver and mesenteric lymph nodes (Supplemental Figure 10G-I). Importantly, following infection, putrescine administration resulted in more disruptive effect on intestinal barrier function compared to vehicle controls, accompanied by higher systemic FITC-dextran influx (Supplemental Figure 10J), elevated trans-epithelial electrical flux (Supplemental Figure 10K, L) and reduced epithelial ZO-1 integrity (Supplemental Figure 11A-C). Furthermore, putrescine-treated mice displayed more remarkable proliferation and apoptosis in the colonic epithelium (Supplemental Figure 11D-G) and enhanced inflammatory responses in the lamina propria, as featured by higher Th17 cell responses with more production of IL-22, IL-17 and interferon-gamma (Supplemental Figure $11 \mathrm{H}-\mathrm{L})$.

RNA-sequencing of sorted EpCAM+ colonic epithelial cells in mice treated with putrescine and taurine. Mice treated with putrescine showed increased expression of genes related to metal binding and oxidative stress, including $m t 1, m t 2, h b b-b t, h b a-a 1, h b a-b 2, M t n d 3$ (Supplemental Figure 12A). 
Genes involved in regulating cytoskeletal organization and contractility, such as malat1, trio, pcm1, dst, Map4k4, cdc42bpg, Arhgap32, Arhgef38, were also upregulated (Supplemental Figure 12A). The differentially expressed genes were enriched in pathways related to the regulation of cell-cell junction and cytoskeleton binding (Supplemental Figure 12B), suggesting that putrescine treatment may induce oxidative stress and regulate the cytoskeletal organization and dynamics in colonic epithelial cells, which subsequently disrupts gut epithelial barrier. We then compared the transcriptional signatures induced by putrescine alone and putrescine combined with taurine. The expression of genes that were elicited by putrescine treatment compared to vehicle control was significantly decreased upon co-administration with taurine (Supplemental Figure 12C), implying a possible rescuing effect of taurine in the oxidative stress and cellular contractility caused by putrescine. 


\section{Supplemental Tables}

Supplemental Table 1. List of epithelial disruptors and stabilizer identified in the screening of humane secreted molecules library.

\section{Epithelial disruptors}

\begin{tabular}{|c|c|c|}
\hline Molecule & & Functional group \\
\hline IL-15 & Interleukin-15 & \multirow{2}{*}{ Mediators of inflammation } \\
\hline CCL-20 & Chemokine ligand 20 & \\
\hline CCL-23 & Chemokine ligand 23 & \multirow{2}{*}{} \\
\hline FGF-1 & Fibroblast growth factor 1 (acidic) & lators of proliferation and morphoge \\
\hline FGF-10 & Fibroblast growth factor 10 & \\
\hline BMP-10 & Bone morphogenetic protein 10 & \multirow{2}{*}{ Vasoconstrictor } \\
\hline UTS-2 & Urotensin 2 & Neuropeptides \\
\hline UCN-1 & Urocortin 1 & \\
\hline UCN-3 & Urocortin 3 & Inductor of erythropoiesis \\
\hline EPO & Erythropoietin \\
\hline
\end{tabular}

\section{Epithelial stabilizers}

\begin{tabular}{|c|c|c|}
\hline Molecule & & Functional group \\
\hline IL-21 & Interleukin-21 & Mediators of inflammations \\
\hline CCL-3 & Chemokine ligand 3 & Regulator of ECM remodeling \\
\cline { 1 - 2 } TIMP-2 & $\begin{array}{c}\text { Tissue metalloprotease } \\
\text { inhibitor 2 }\end{array}$ & Inductor of apoptosis \\
\hline FasLG & Fas ligand & \\
\hline
\end{tabular}


Supplemental Table 2. List of microbiota-associated metabolites used for screening.

Metabolites abundant in dysbiotic intestine

\begin{tabular}{|c|c|c|}
\hline Biochemical Name & Biosynthetic pathway & $\begin{array}{c}\text { eased/ healt } \\
\text { ratio }\end{array}$ \\
\hline putrescine & Polyamine Metabolism & 145.20 \\
\hline N-acetyltryptophan & Tryptophan Metabolism & 65.61 \\
\hline 3-(3-sulfooxyphenyl)propanoic acid & Phenylalanine and Tyrosine Metabolism & 54.29 \\
\hline N-acetylputrescine & Polyamine Metabolism & 48.58 \\
\hline histamine & Histidine Metabolism & 44.18 \\
\hline choline phosphate & Phospholipid Metabolism & 25.63 \\
\hline N-acetylproline & Urea cycle; Arginine and Proline Metabolism & 16.58 \\
\hline spermine & Polyamine Metabolism & 15.70 \\
\hline biliverdin & Hemoglobin and Porphyrin Metabolism & 11.40 \\
\hline adenosine 5'-monophosphate (AMP) & Purine Metabolism, Adenine containing & 8.49 \\
\hline gamma-aminobutyrate (GABA) & Glutamate & 7.80 \\
\hline
\end{tabular}

Metabolites abundant in healthy intestine

\begin{tabular}{|c|c|c|}
\hline Biochemical Name & Biosynthetic pathway & $\begin{array}{c}\text { eased/ healt } \\
\text { ratio }\end{array}$ \\
\hline tryptamine & Tryptophan Metabolism & 0.08 \\
\hline maltose & Glycogen Metabolism & 0.16 \\
\hline taurodeoxycholate & Secondary Bile Acid Metabolism & 0.16 \\
\hline S-adenosylmethionine (SAM) & Methionine, Cysteine, SAM and Taurine Metabolism & 0.18 \\
\hline taurine & Methionine, Cysteine, SAM and Taurine Metabolism & 0.28 \\
\hline pinitol & Inositol Metabolism & 0.35 \\
\hline glutarate (pentanedioate) & Lysine Metabolism & 0.36 \\
\hline sebacate (decanedioate) & Fatty Acid, Dicarboxylate & 0.39 \\
\hline chenodeoxycholate & Primary Bile Acid Metabolism & 0.47 \\
\hline dodecanedioate & Fatty Acid, Dicarboxylate & 0.48 \\
\hline L-homoserine & Glycine, Serine and Threonine Metabolism & 0.49 \\
\hline ethylmalonate & Leucine, Isoleucine and Valine Metabolism & 0.50 \\
\hline undecanedioate & Fatty Acid, Dicarboxylate & 0.54 \\
\hline flavin mononucleotide (FMN) & Riboflavin Metabolism & 0.56 \\
\hline
\end{tabular}


Supplemental Table 3. List of biologically active compounds identified as stabilizers or disruptors in the screening of Selleck library.

\section{Pharmacologically active epithelial stabilizers}

DNA damage

\begin{tabular}{|c|c|}
\hline Mitoxantrone Hydrochloride & DNA intercalating agent \\
\hline Daunorubicin $\mathrm{HCl}$ (Daunomycin $\mathrm{HCl}$ ) & DNA intercalating agent \\
\hline Epirubicin Hydrochloride & DNA intercalating agent \\
\hline Idarubicin HCl & DNA intercalating agent \\
\hline Topotecan HCl & Topoisomerase inhibitor \\
\hline (S)-(+)-Camptothecin & Topoisomerase inhibitor \\
\hline 10-Hydroxycamptothecin & PARP inhibitor \\
\hline AZD2461 & Inhibitor of purine synthesis \\
\hline Mycophenolic acid &
\end{tabular}

\section{Cell cycle arrest}

\begin{tabular}{|c|c|}
\hline Flavopiridol hydrochloride & CDK inhibitor \\
\hline Dinaciclib (SCH727965) & CDK inhibitor \\
\hline SNS-032 (BMS-387032) & CDK-2 inhibitor \\
\hline AT7519 & multi-CDK inhibitor \\
\hline Abemaciclib (LY2835219) & CDK4/6 inhibitor \\
\hline CHIR-124 & Chk1 inhibitor \\
\hline NH125 & eEF-2 kinase inhibitor \\
\hline RITA (NSC 652287) & MDM2 inhibitor \\
\hline
\end{tabular}

Tyrosine kinases inhibitors

\begin{tabular}{|c|c|}
\hline VX-702 & p38 MAPK inhibitor \\
\hline CP 673451 & PDGFR inhibitor \\
\hline PRT062607 (P505-15, ) HCl & Syk inhibitor \\
\hline JAK3 Inhibitor VI & Raf and ERK inhibitor \\
\hline GDC-0879 & . \\
\hline
\end{tabular}

NF-KB inhibitors

\begin{tabular}{|c|c|}
\hline Triptolide & NF- $\kappa B$ inhibitor \\
\hline Bardoxolone Methyl & IKB kinase/NF- $\kappa B$ inhibitor \\
\hline
\end{tabular}

PI3K/Akt inhibitors

\begin{tabular}{|c|l|}
\hline A-674563 & Akt1 inhibitor \\
\hline PIK-75 & PI3K inhibitor \\
\hline GSK2126458 & PI3K inhibitor \\
\hline
\end{tabular}


Retinoic acid derivates

\begin{tabular}{|c|c|}
\hline Acitretin & Retinoic acid derivate \\
\hline Tazarotene (Avage) & Retinoic acid derivate \\
\hline Bexarotene & Retinoic acid derivate \\
\hline All-trans Retinoic Acid (Tretinoin) & Retinoic acid \\
\hline
\end{tabular}

Smooth muscle relaxants

\begin{tabular}{|c|c|}
\hline Tetrahydropapaverine hydrochloride & Phosphodiesterase (PDE) inhibitor \\
\hline Icariin & PDE 5 inhibitor \\
\hline Tetrandrine (Fanchinine) & Ca++ channel blocker \\
\hline Alverine Citrate & Ca++ channel blocker \\
\hline 5-hydroxymethyl tolterodine & Muscarinic receptor antagonist \\
\hline
\end{tabular}

Others (pharmacologically active)

\begin{tabular}{|c|c|}
\hline Salicin (Salicoside) & COX inhibitor \\
\hline Lorcaserin HCl & 5-HT (Serotonin) Receptor agonist \\
\hline Vortioxetine hydrobromide & 5-HT Receptor agonist/SSRI \\
\hline L-Thyroxine & Thyroid hormone \\
\hline Carbimazole & Thyroid peroxidase inhibitor \\
\hline Shikimic acid (Shikimate) & Precursor of aromatic amino acids \\
\hline Ketoconazole & Antifungal drug \\
\hline Enrofloxacin & Bacterial topoisomerase inhibitor \\
\hline
\end{tabular}

Poorly characterized/plant-derived/food supplements

\begin{tabular}{|c|c|}
\hline Apocynin (Acetovanillone) & NADPH oxidase inhibitor \\
\hline Naringin (Naringoside) & Flavonoid, P450 inhibitor, antioxidant \\
\hline Quercetin dihydrate (Sophoretin) & Flavonoid, antioxidant \\
\hline Chrysin & Flavonoid \\
\hline Kaempferol & Flavonoid \\
\hline Sesamin (Fagarol) & Food supplement \\
\hline Vanillylacetone & Antioxidant \\
\hline
\end{tabular}


Pharmacologically active epithelial disruptors:

Cell cycle arrest

\begin{tabular}{|c|c|}
\hline MLN8237 (Alisertib) & Aurora Kinase inhibitor \\
\hline Danusertib (PHA-739358) & Aurora Kinase, Bcr-Abl, c-RET, FGFR inhibitor \\
\hline Rigosertib (ON-01910) & PLK/Src inhibitor \\
\hline PHA-848125 & CDK/TRKA inhibitor \\
\hline NVP-AEW541 & IGF-1R inhibitor \\
\hline NVP-ADW742 & IGF-1R inhibitor \\
\hline Gemcitabine & Nucleoside analog \\
\hline
\end{tabular}

Regulation of transcription

\begin{tabular}{|c|c|}
\hline LY2109761 & TGF- R/Smad inhibitor \\
\hline TG101348 (SAR302503) & JAK inhibitor \\
\hline CEP33779 & JAK inhibitor \\
\hline LY2784544 & JAK inhibitor \\
\hline Baricitinib (LY3009104) & JAK inhibitor \\
\hline CI994 (Tacedinaline) & HDAC inhibitor \\
\hline Lenalidomide & Transcriptional regulation \\
\hline
\end{tabular}

\section{PI3K/Akt inhibitors}

\begin{tabular}{|c|c|}
\hline AT7867 & Akt inhibitor \\
\hline GDC-0068 & Akt inhibitor \\
\hline PF-04691502 & PI3K/mTOR inhibitor \\
\hline
\end{tabular}

Cytoskeleton dynamics

\begin{tabular}{|c|c|}
\hline Lexibulin (CYT997) & Mt depolymerization \\
\hline NPI-2358 (Plinabulin) & Mt depolymerization \\
\hline Epothilone A & Mt stabilization similar to taxanes \\
\hline Nocodazole & Mt depolymerization \\
\hline Vincristine & Mt depolymerization \\
\hline KX2-391 & Src inhibitor/ MT depolymerization \\
\hline GSK 269962 & ROCK inhibitor \\
\hline RKI-1447 & ROCK inhibitor \\
\hline
\end{tabular}

Others (pharmacologically active)

\begin{tabular}{|c|c|}
\hline SCH 79797 & PAR1 antagonist \\
\hline Domperidone (Motilium) & Dopamine D2 receptor antagonist \\
\hline Puerarin (Kakonein) & 5-HT antagonist \\
\hline
\end{tabular}


Poorly characterized/plant-derived/food supplements

\begin{tabular}{|c|c|}
\hline Dioscin (Collettiside III) & Saponin \\
\hline Berberine Hydrochloride & Plant alkaloid \\
\hline Indole-3-carbinol & Food-supplement \\
\hline
\end{tabular}



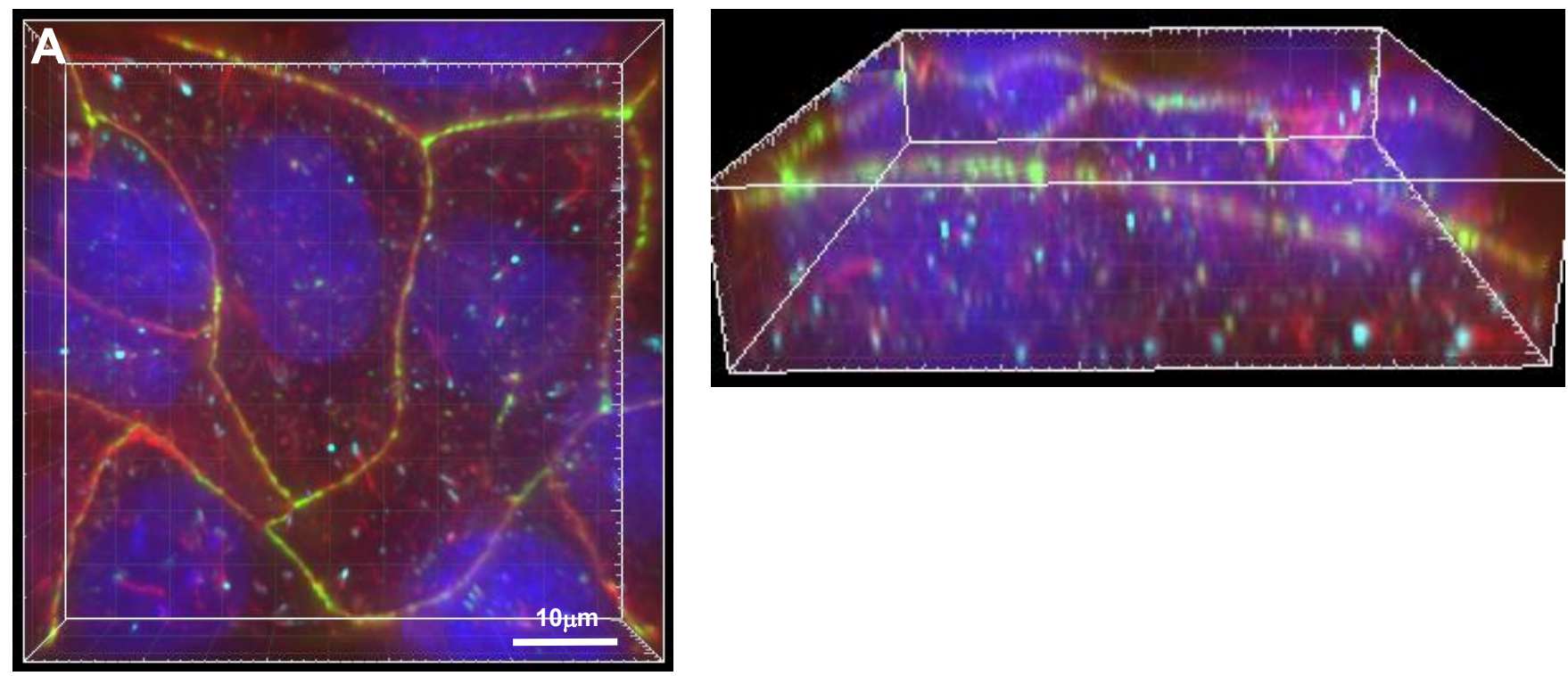

Tight junctions

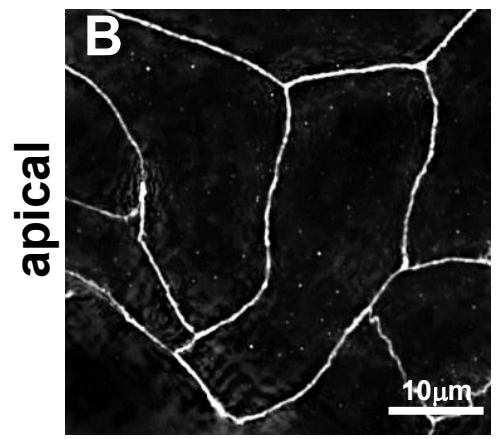

\section{Focal adhesions}
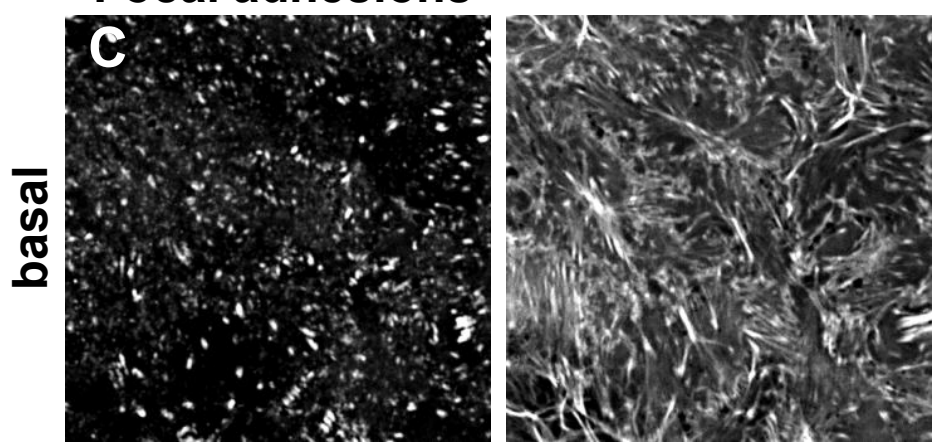

\section{Segmented FA}
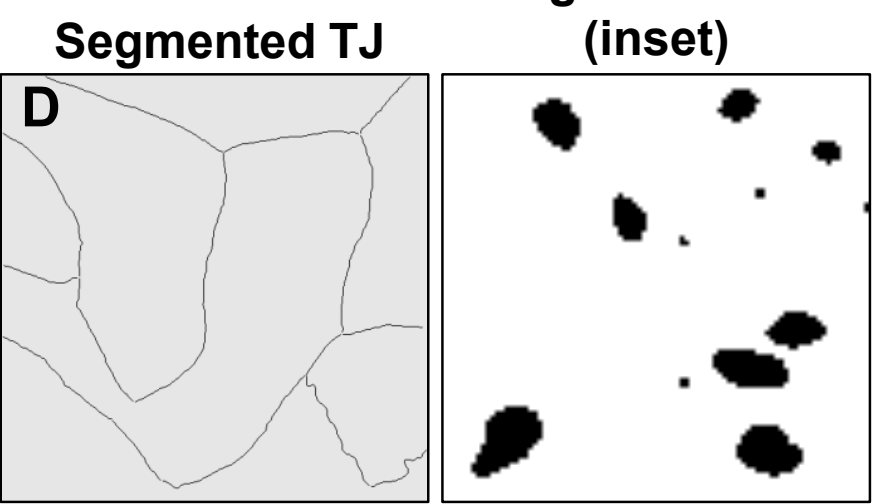

merge

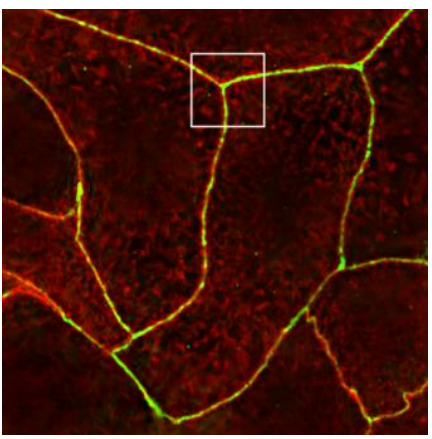

inset

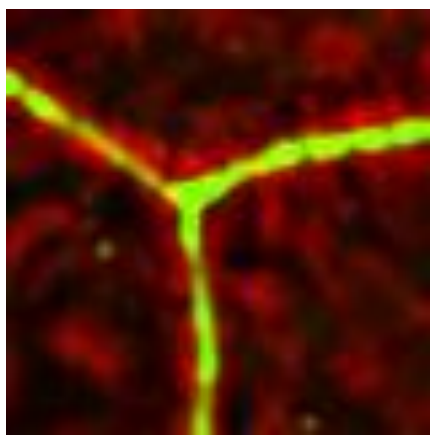

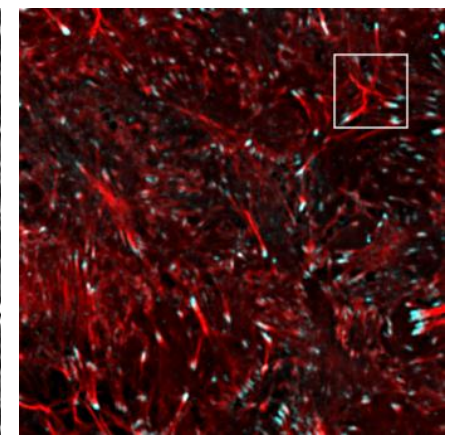

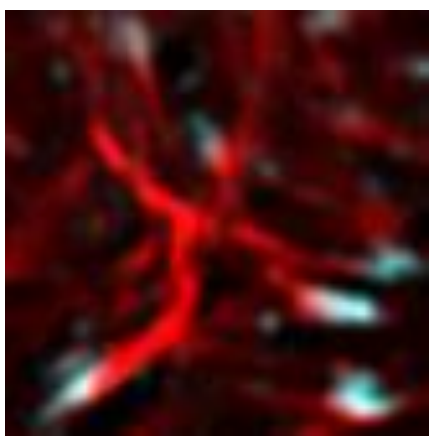

Supplemental figure 1. 
A Tight Focal

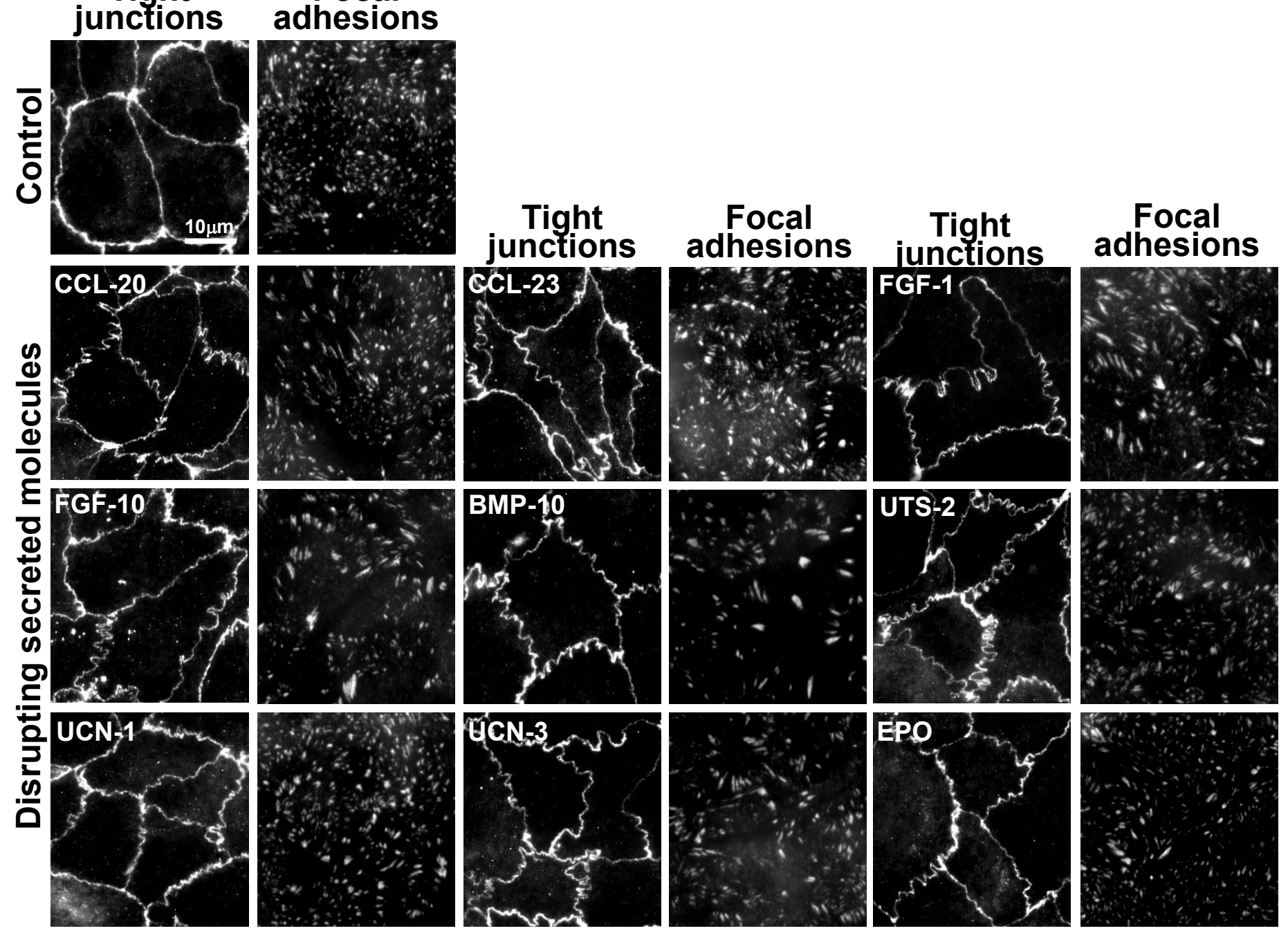

B

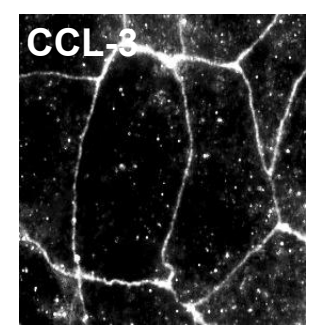

C

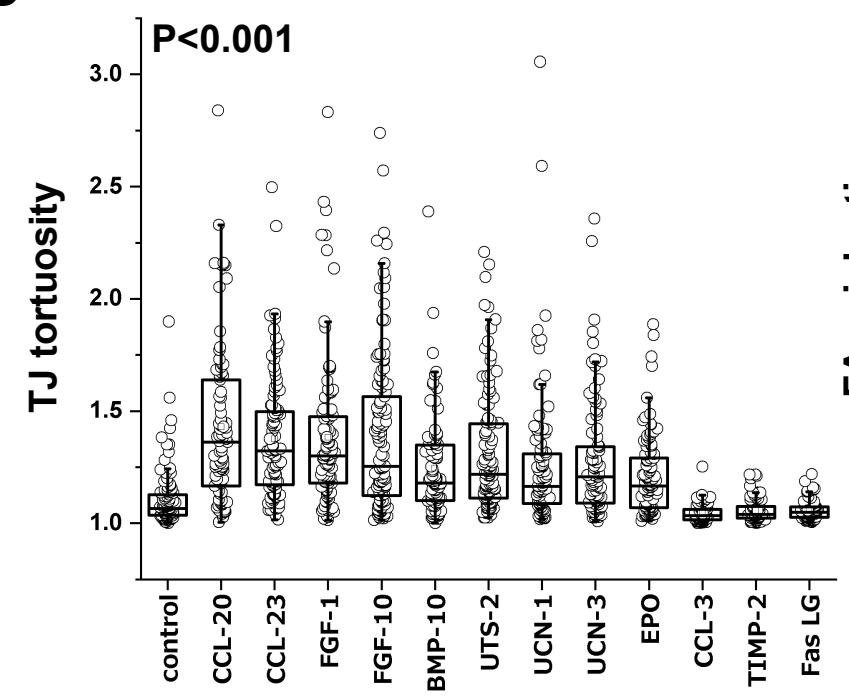

Stabilizing secreted molecules
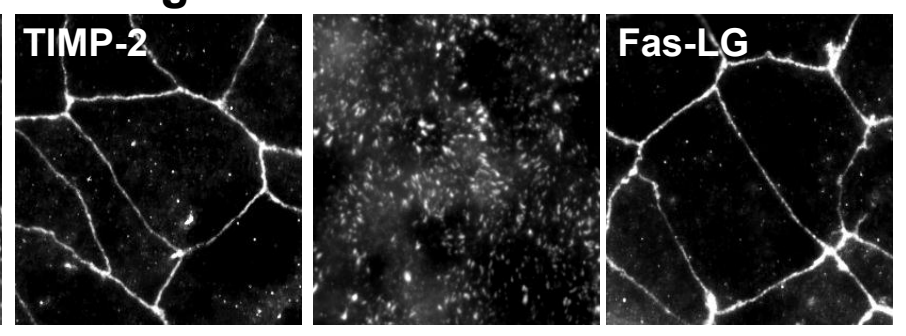

D

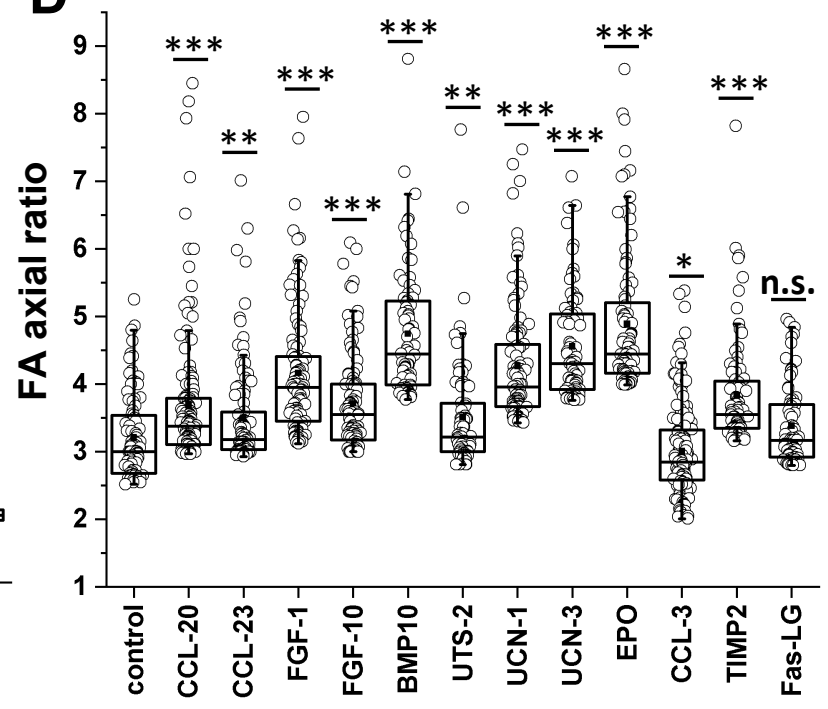

Supplemental figure 2 
Disruptive metabolites

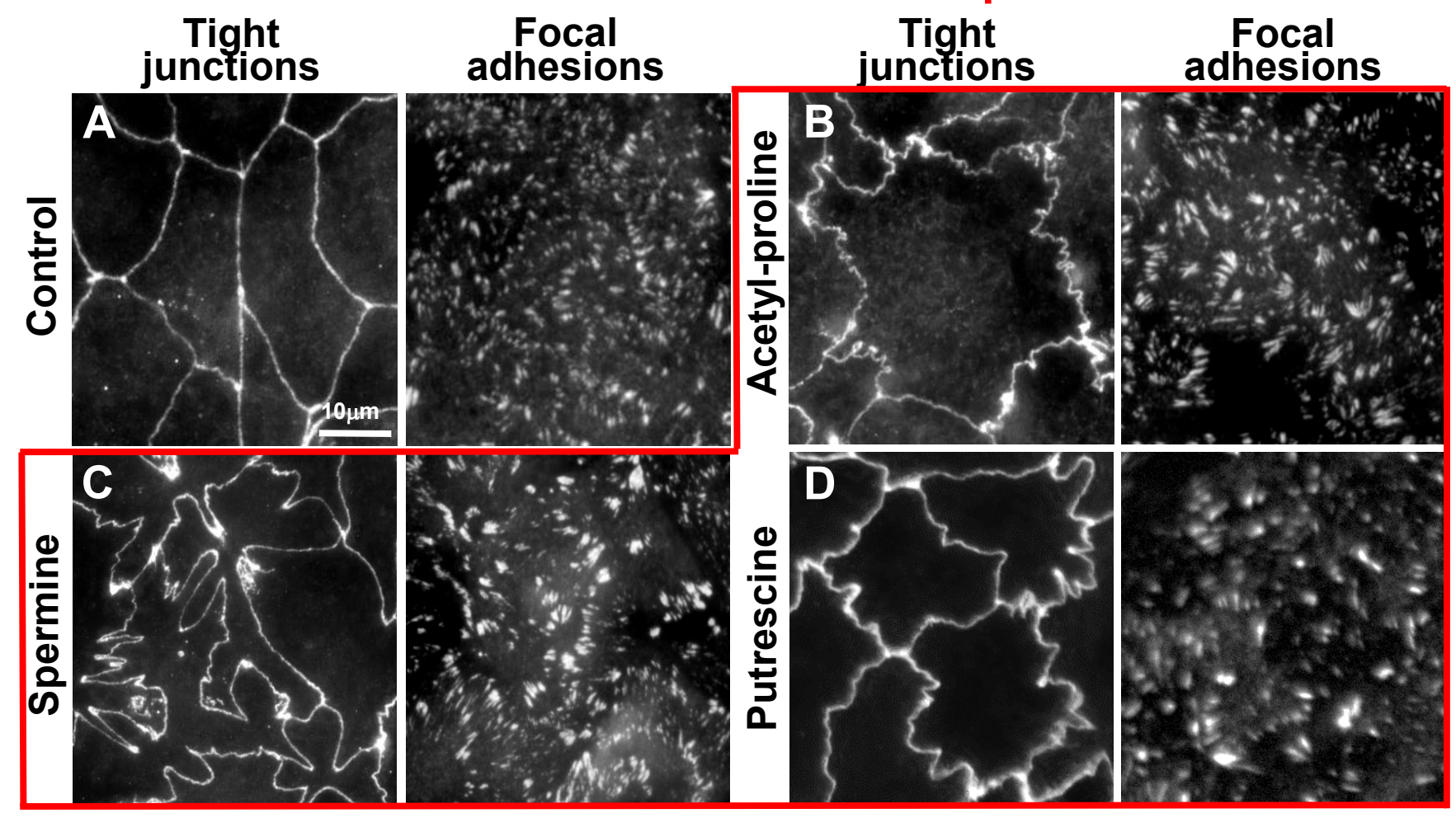

Stabilizing metabolites
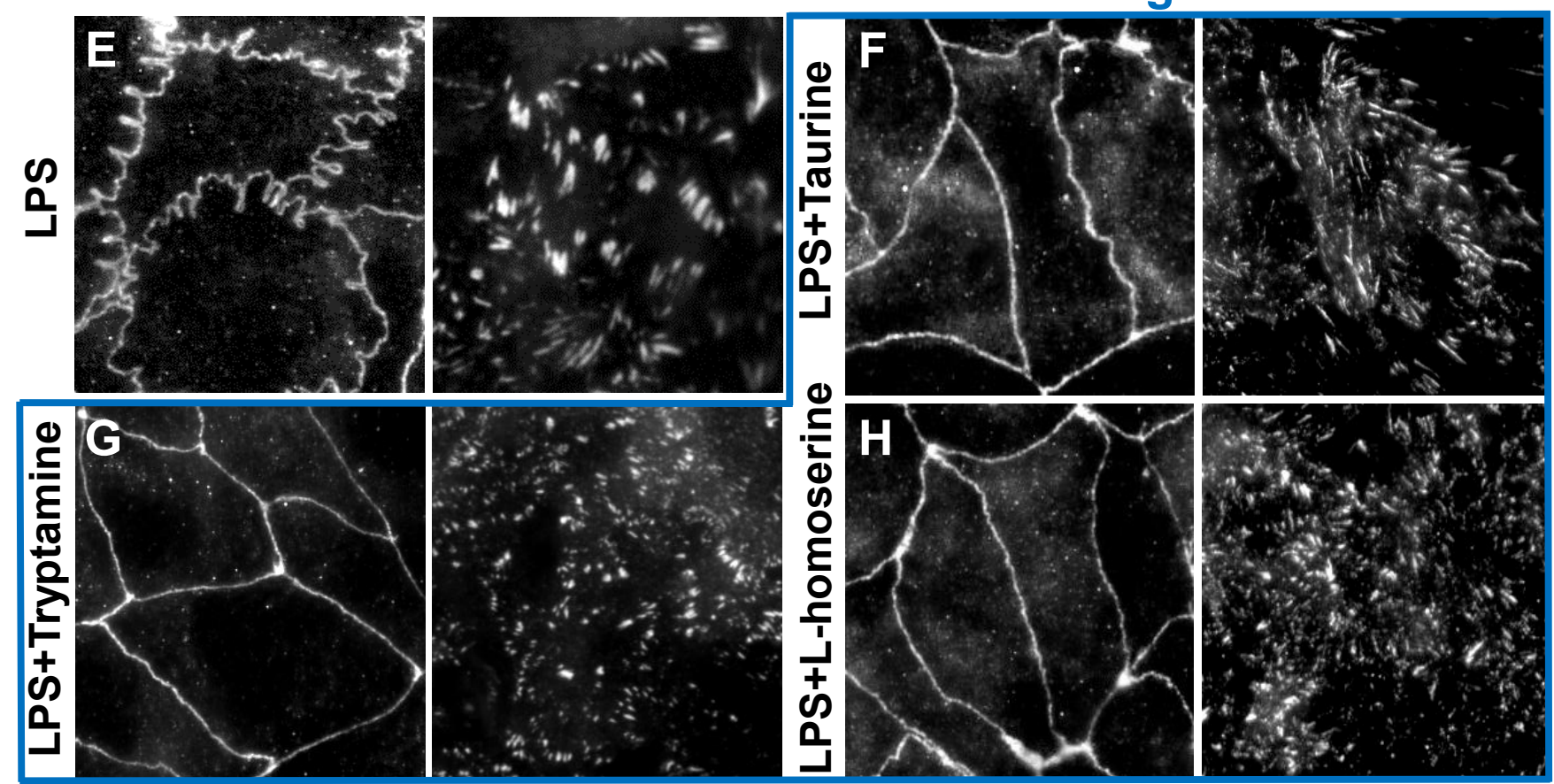

Supplemental figure 3 . 
A LPS+histamine
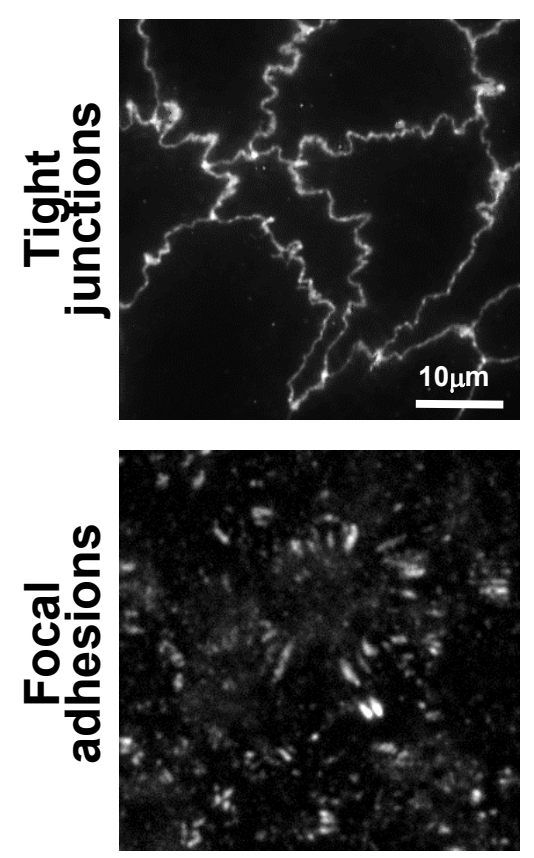

B
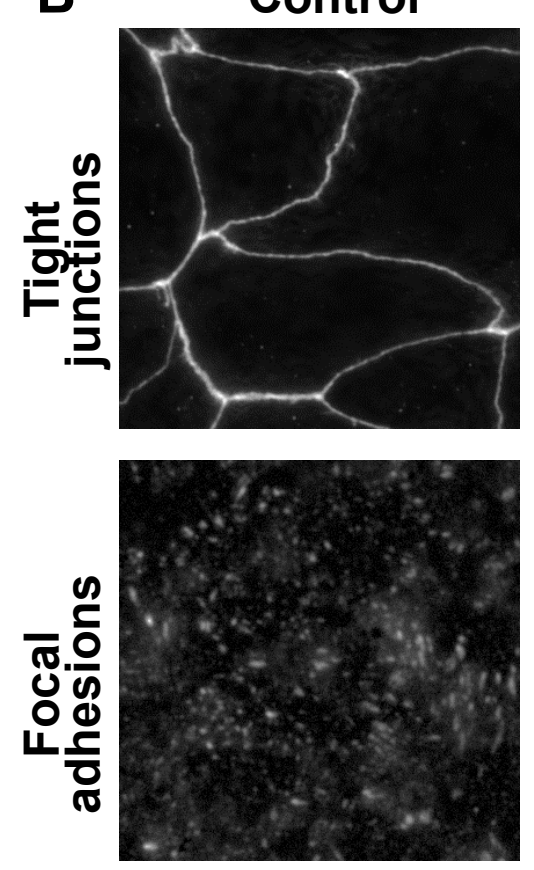

Flavopiridol
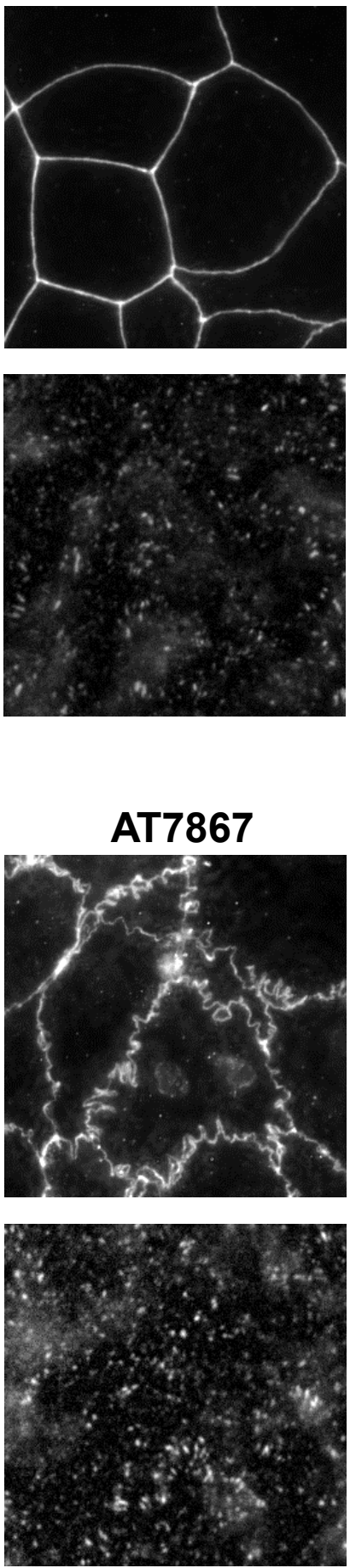

\section{Daunorubicin}
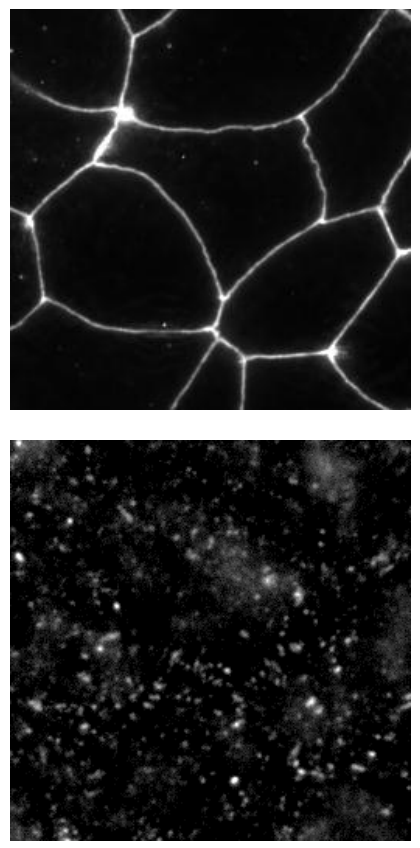

Vincristine
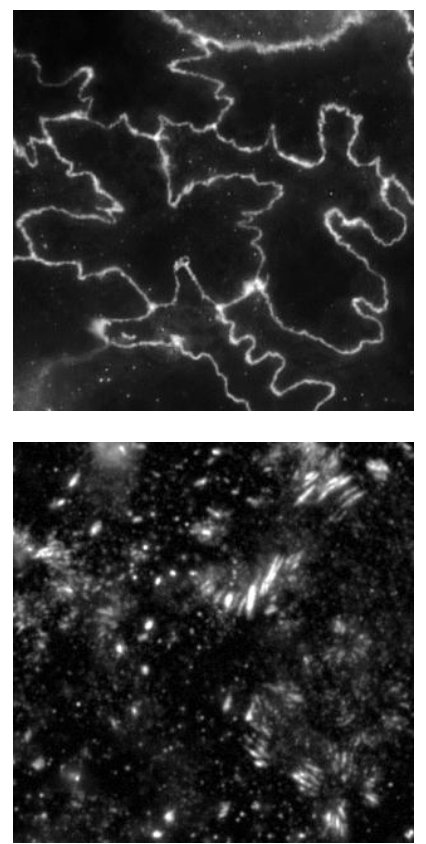

Supplemental figure 4 


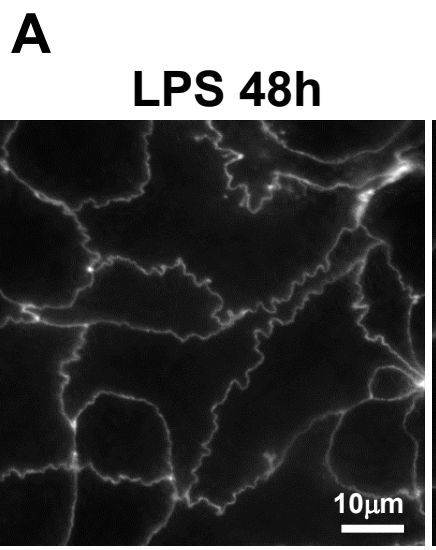

24h LPS; 24h washout 24h LPS;

24h LPS;

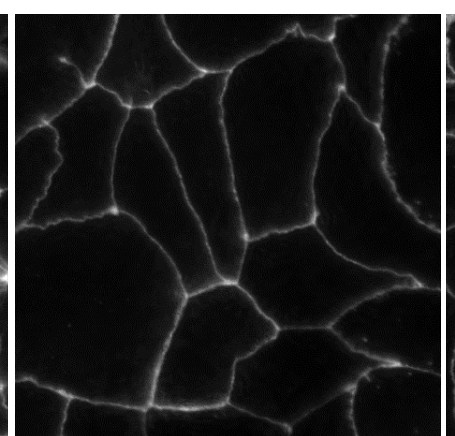

24h His;

His 48h

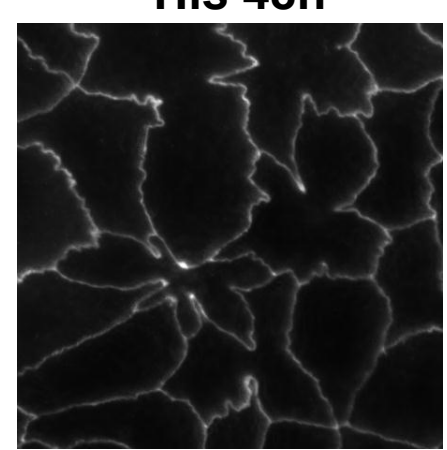

$24 \mathrm{~h}$ washout 24h LPS+Tau 24h His;

24h His; 24h His+Tau

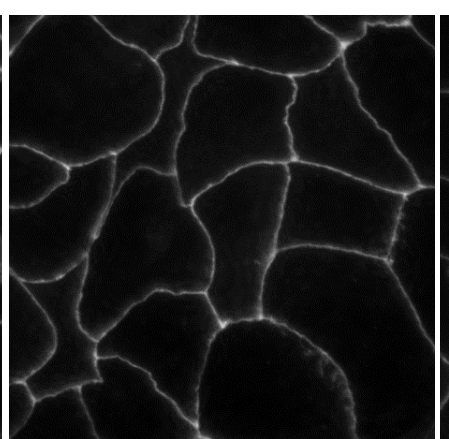

24h LPS+Trypt

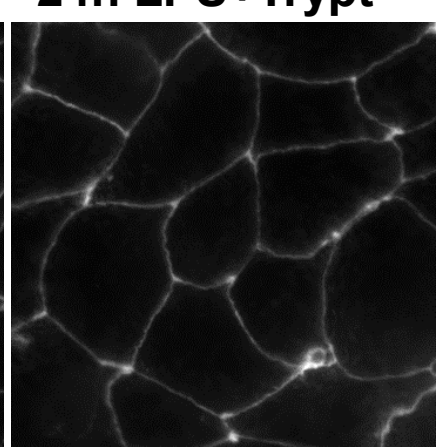

24h Putr;

Putr 48h

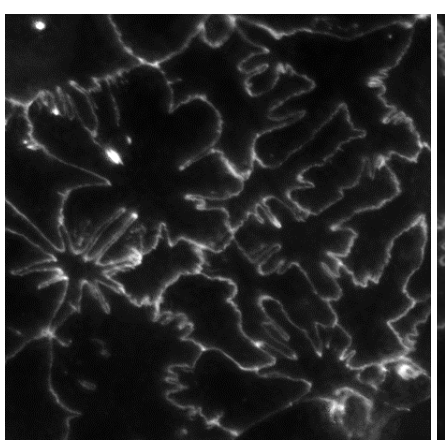

B

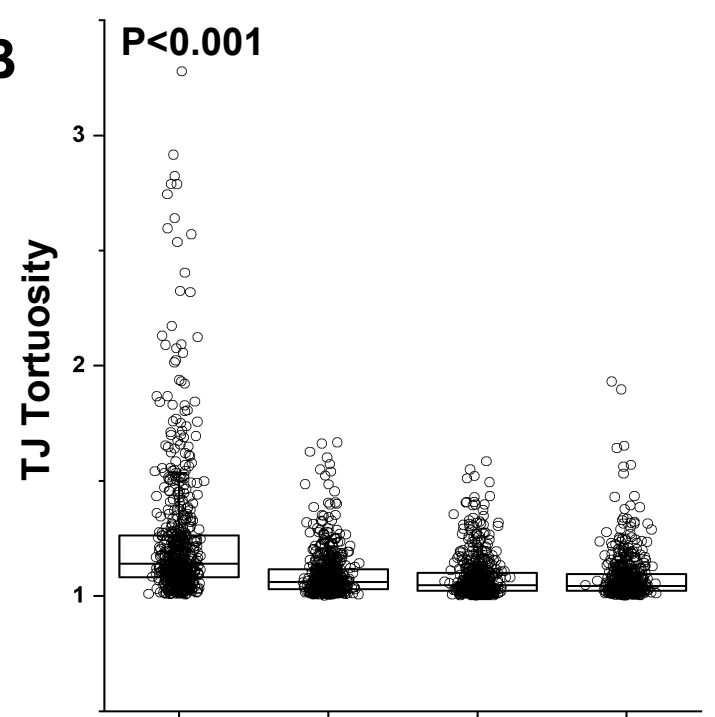

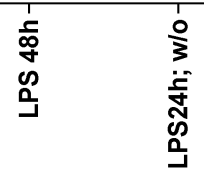

Supplemental figure 5 24h His+Trypt

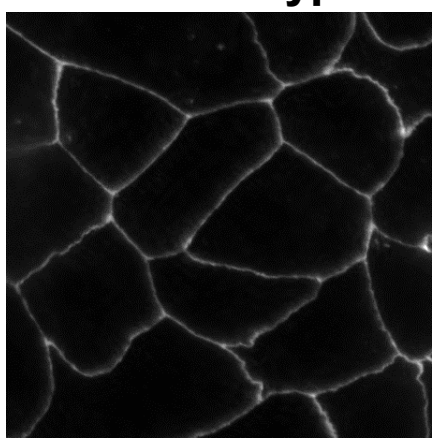

24h Putr; 24h Trypt
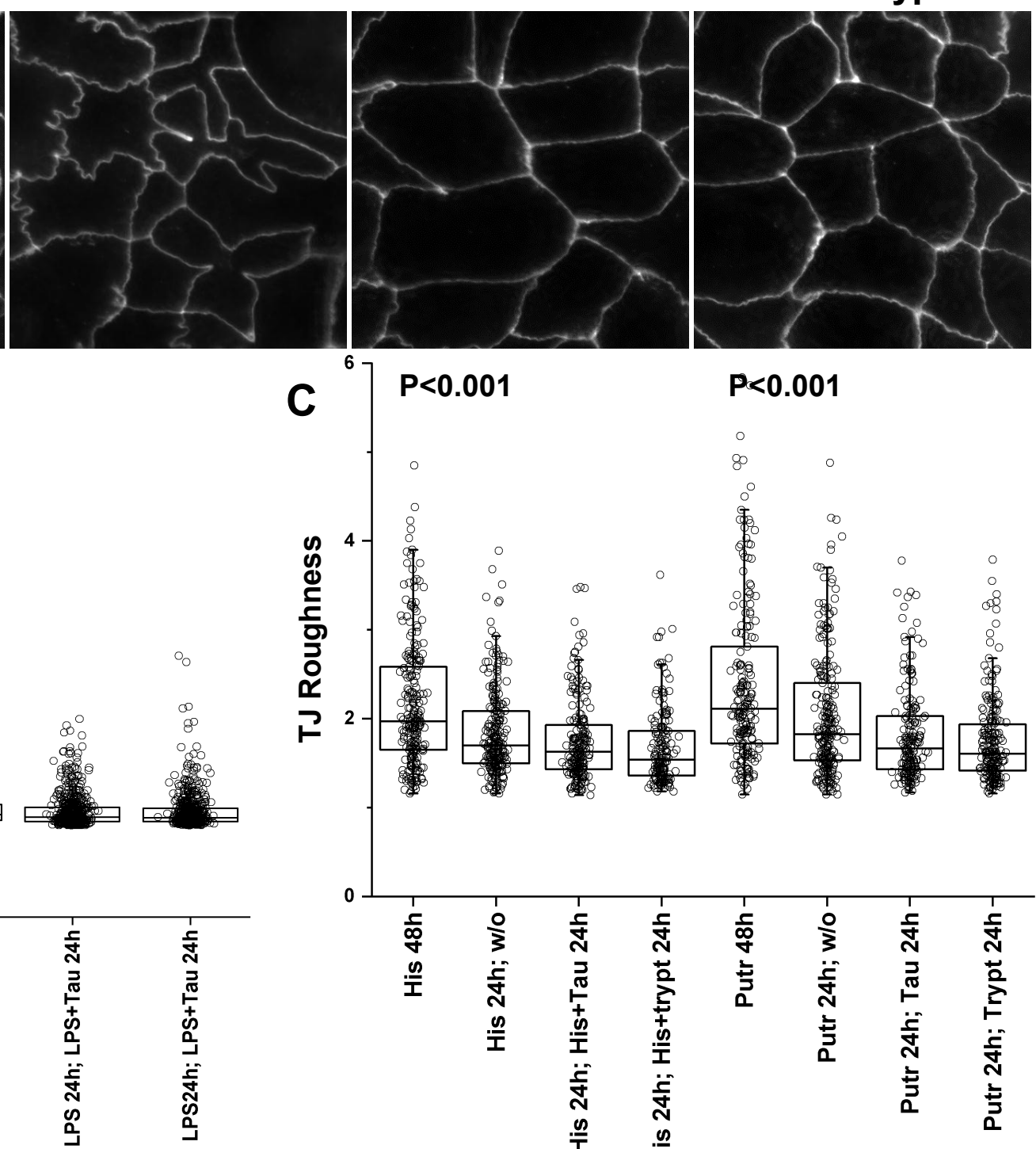

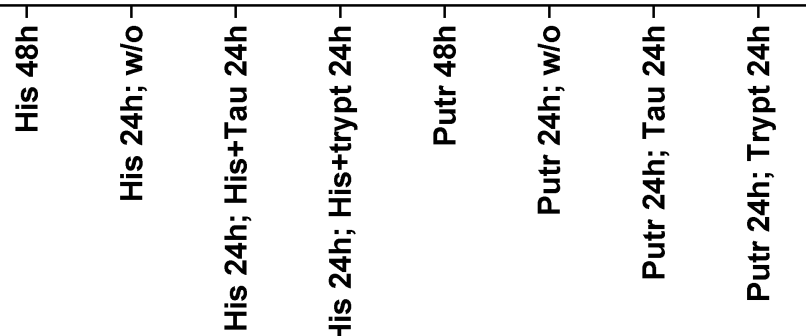




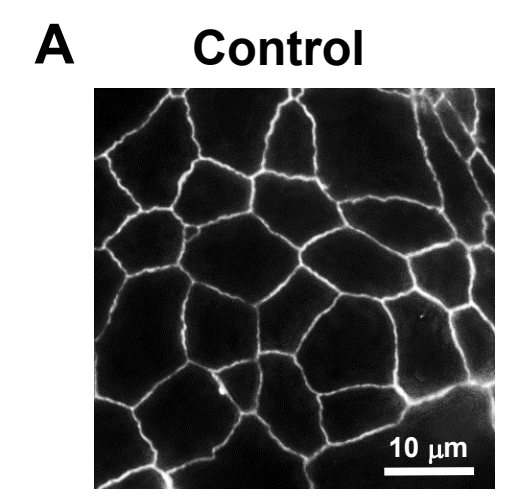

Putrescine

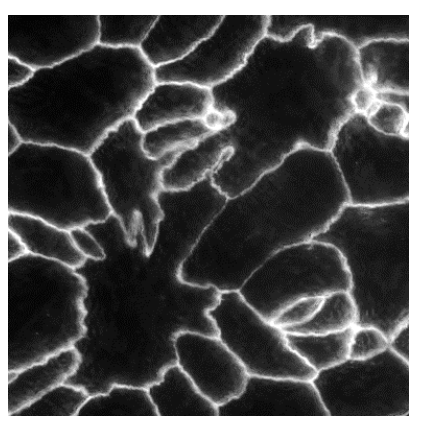

B

B $r<<t$

돌

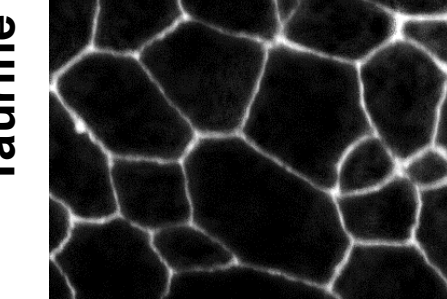

C

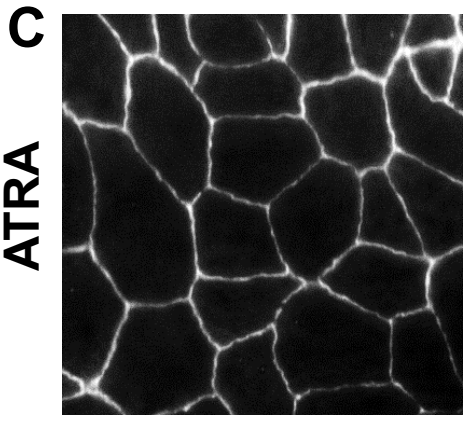

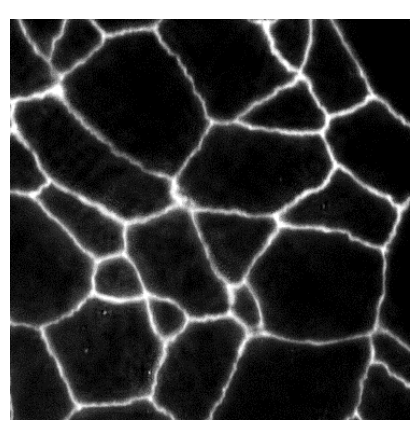

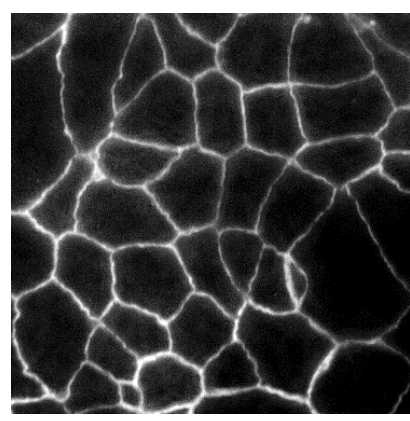

Histamine
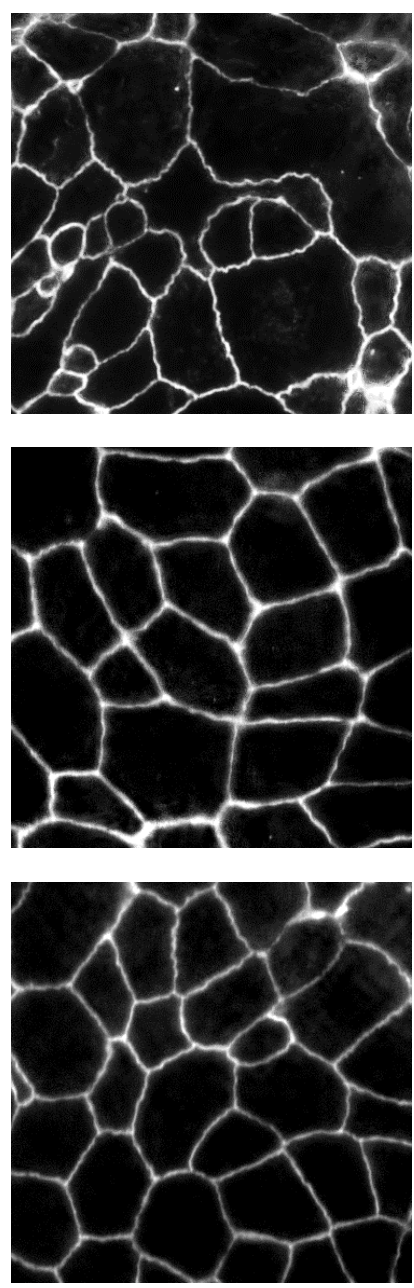

LPS
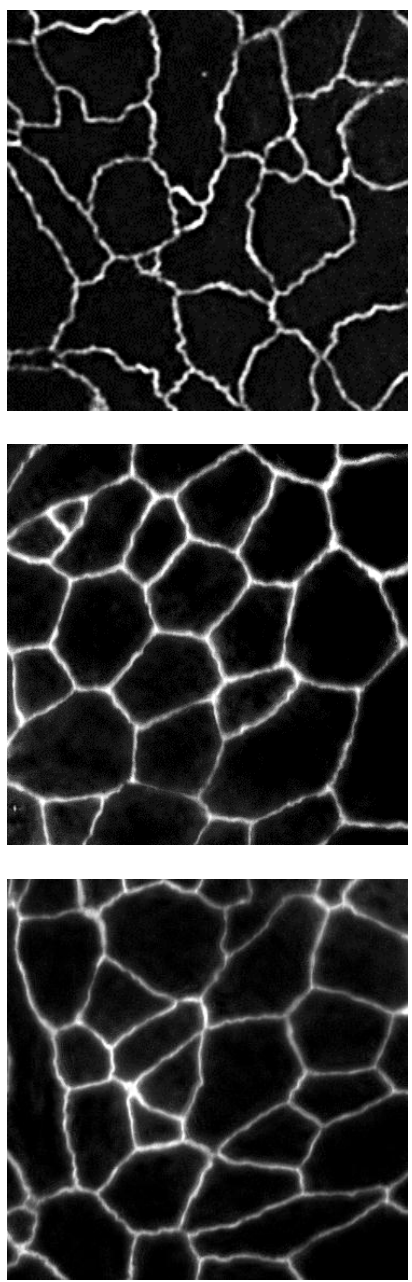
A

Control Caly A 5nM 30 min Caly A 5nM 1h

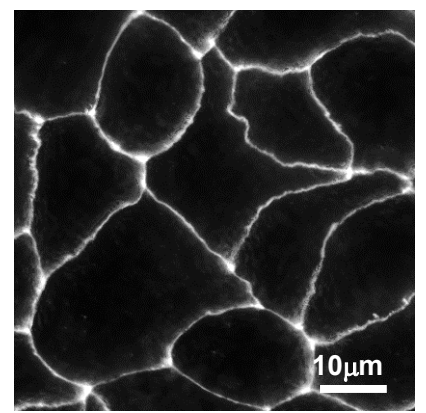

Caly A10nM 30 min Caly A 10nM 1h

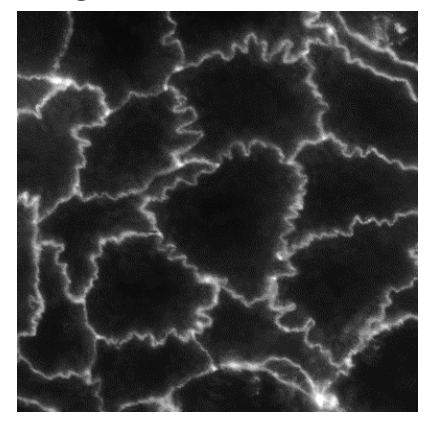

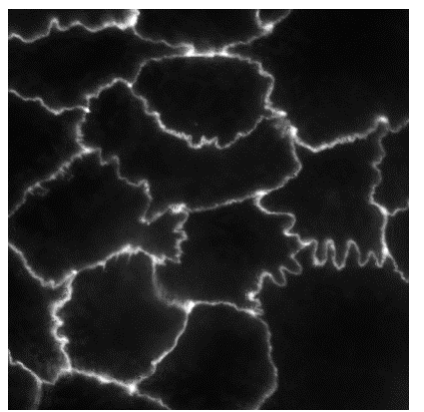

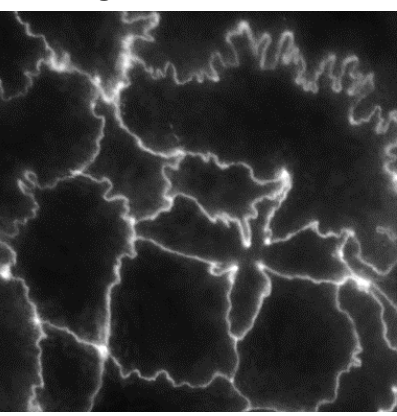

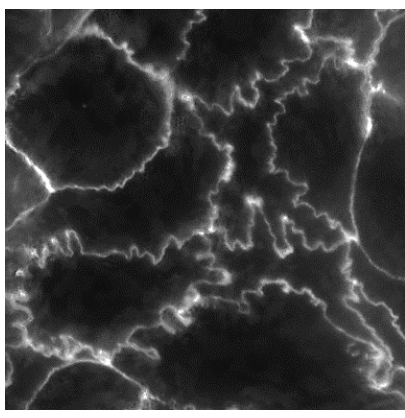

Caly A10nM 2h Caly A 20nM 30min

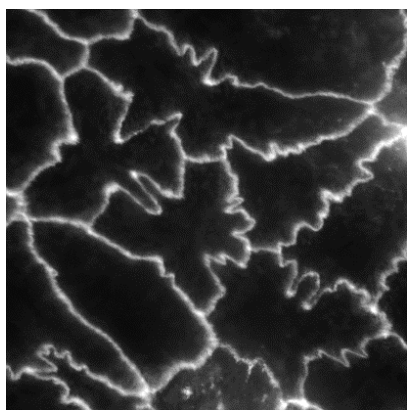

Caly A 5nM $2 \mathrm{~h}$
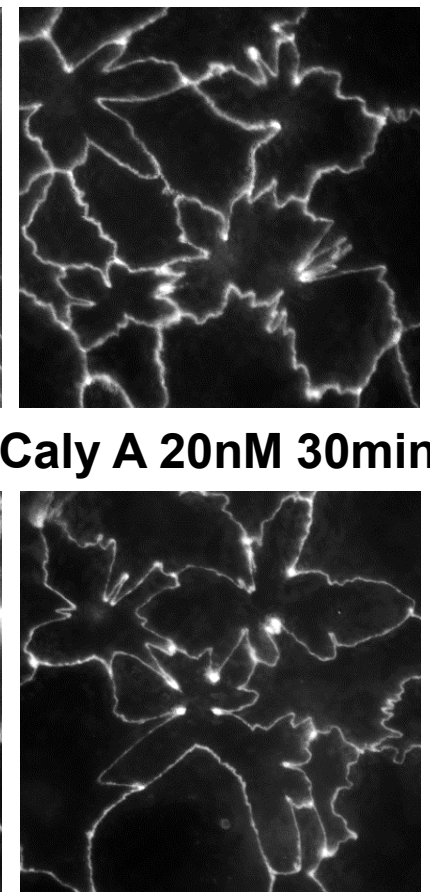

B

Caly A + Tau 1h co-incubation

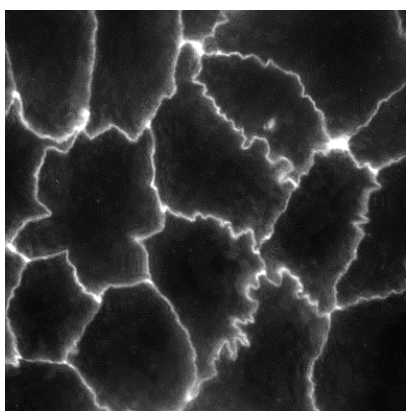

Caly A + Trypt 1h co-incubation $1 \mathrm{~h}$ Caly A + Trypt
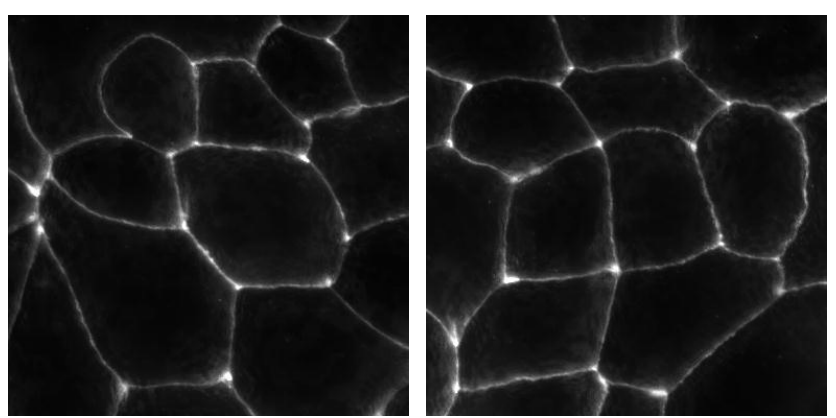

Caly A + ATRA 1h Caly A + Tau

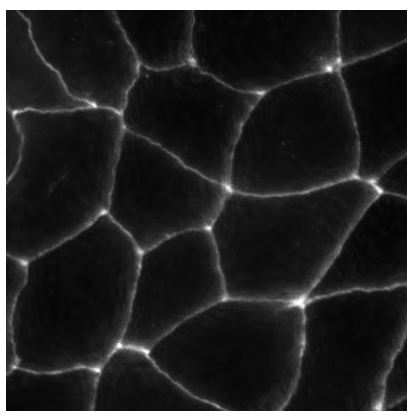

24h Trypt h co-incubation

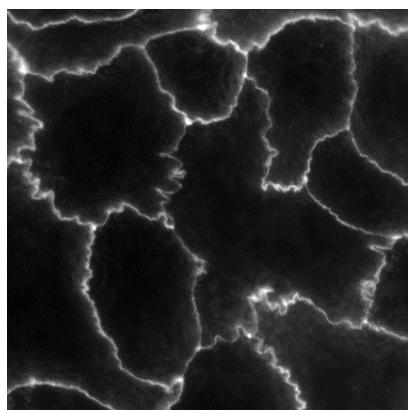

24h ATRA 1h Caly A + ATRA

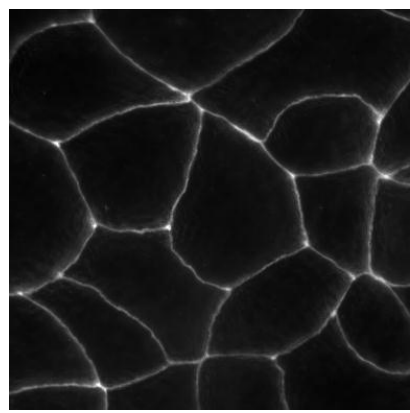

Supplemental figure 7 
A

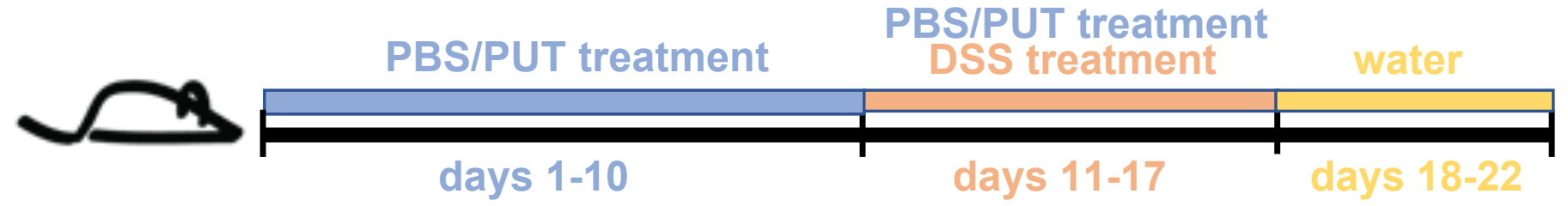

B

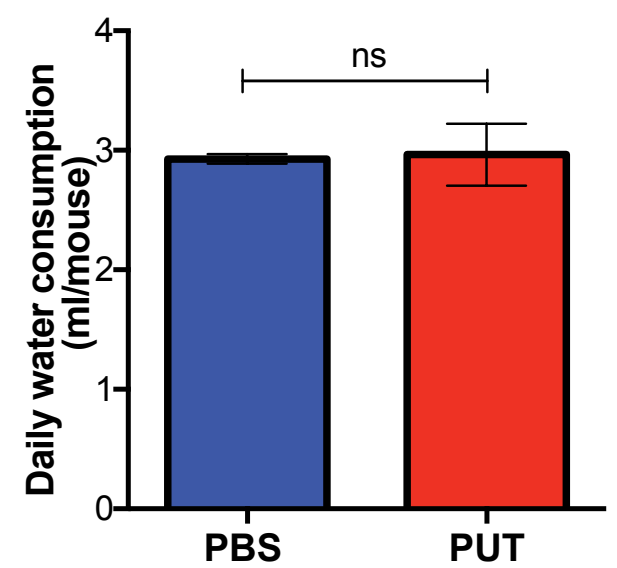

E
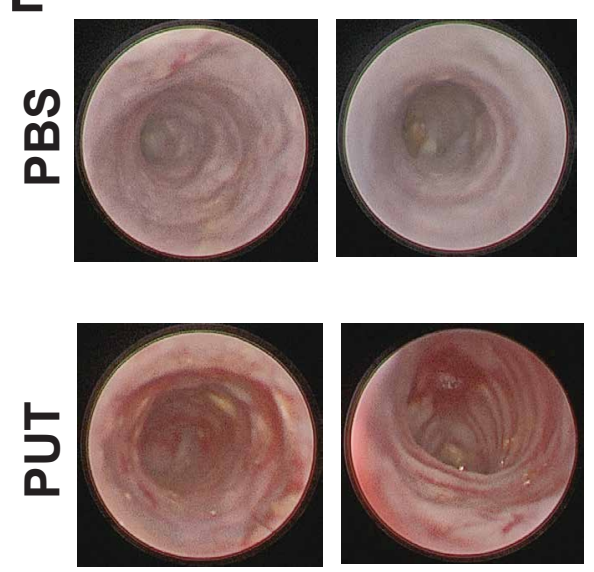

H

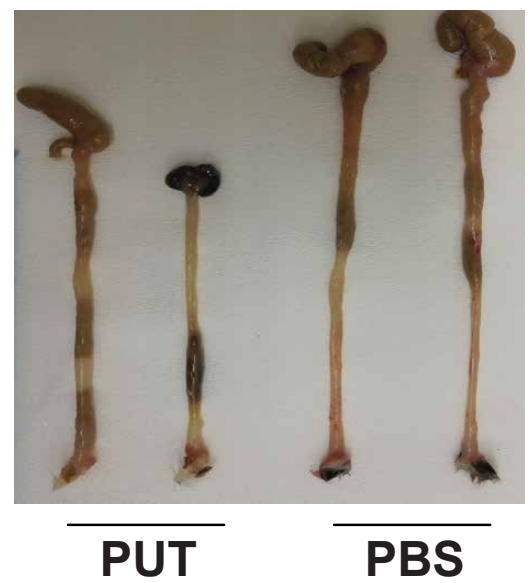

C

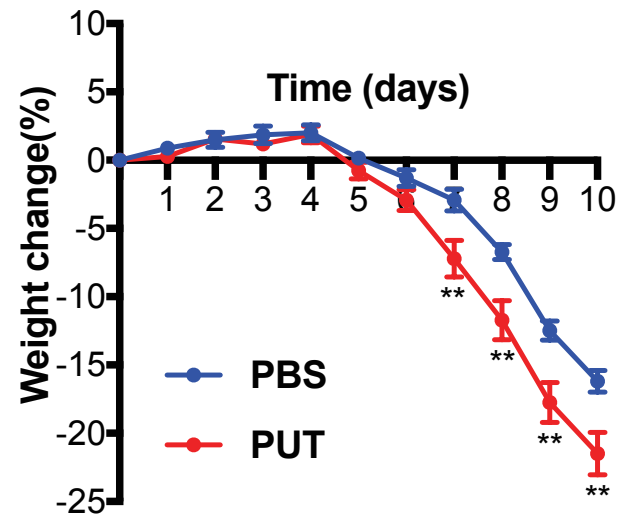

F

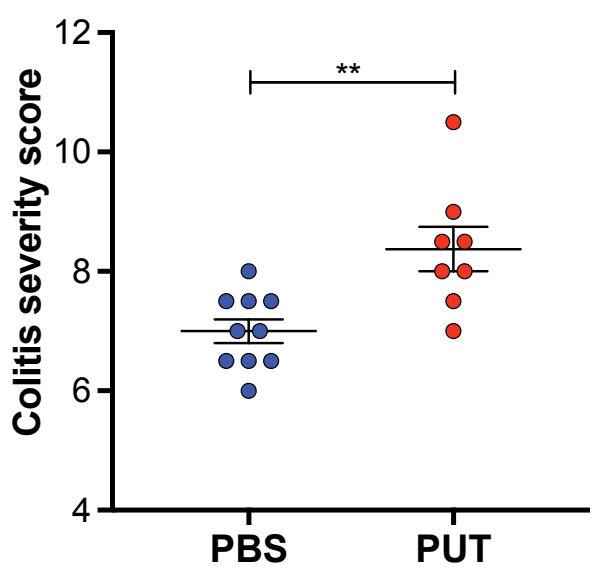

I

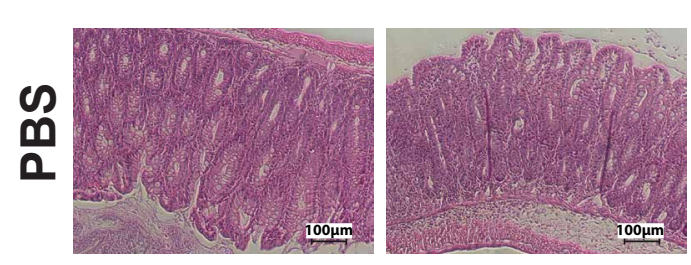

$\frac{5}{2}$

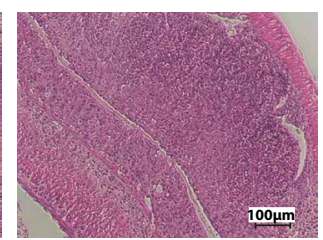

D

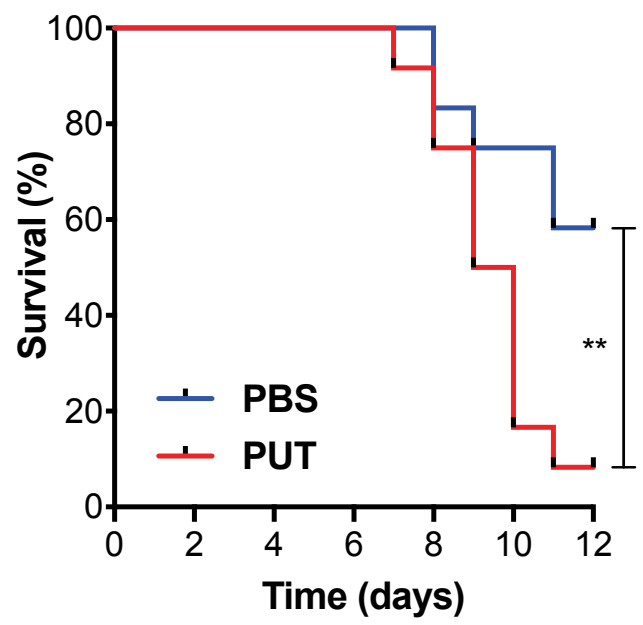

G

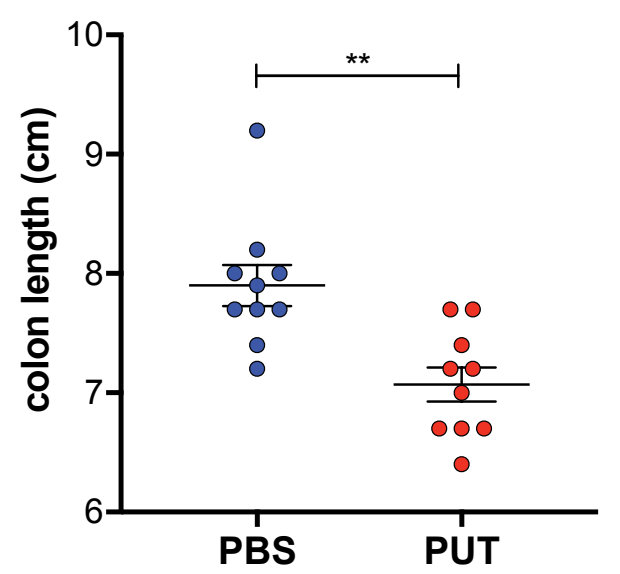

J

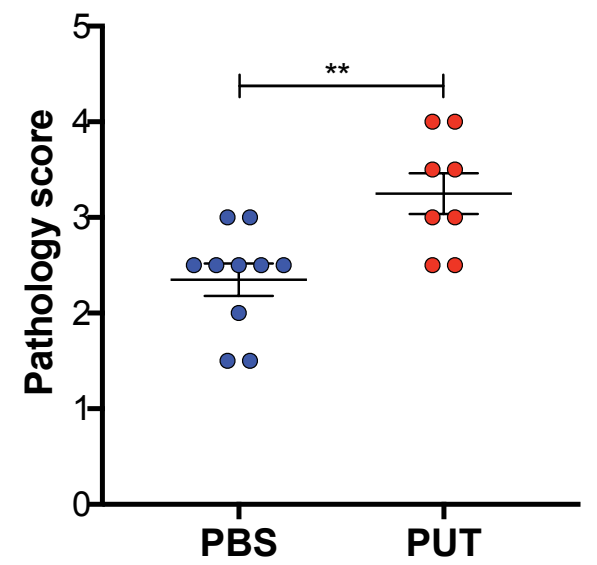



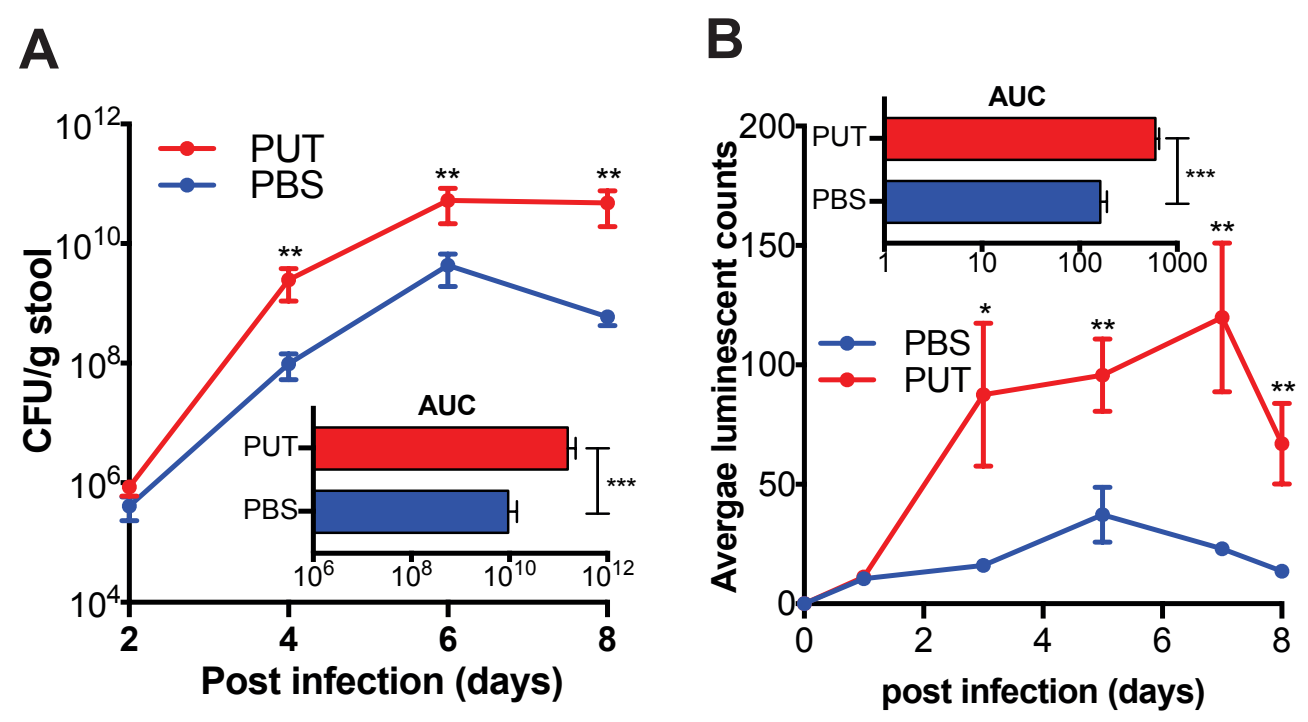

D

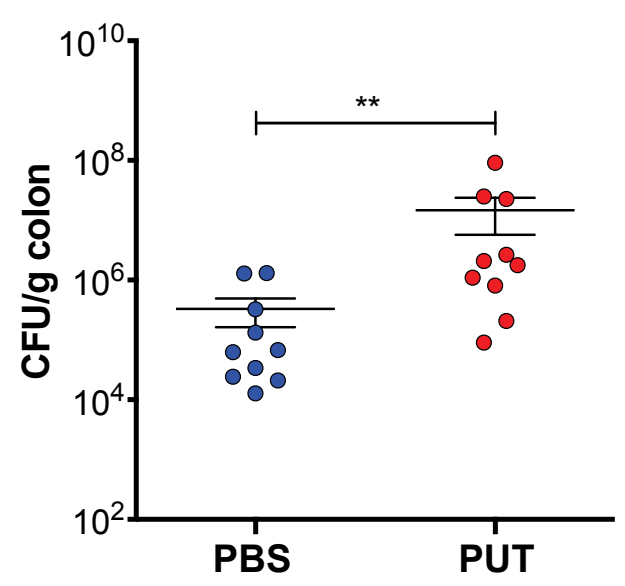

G
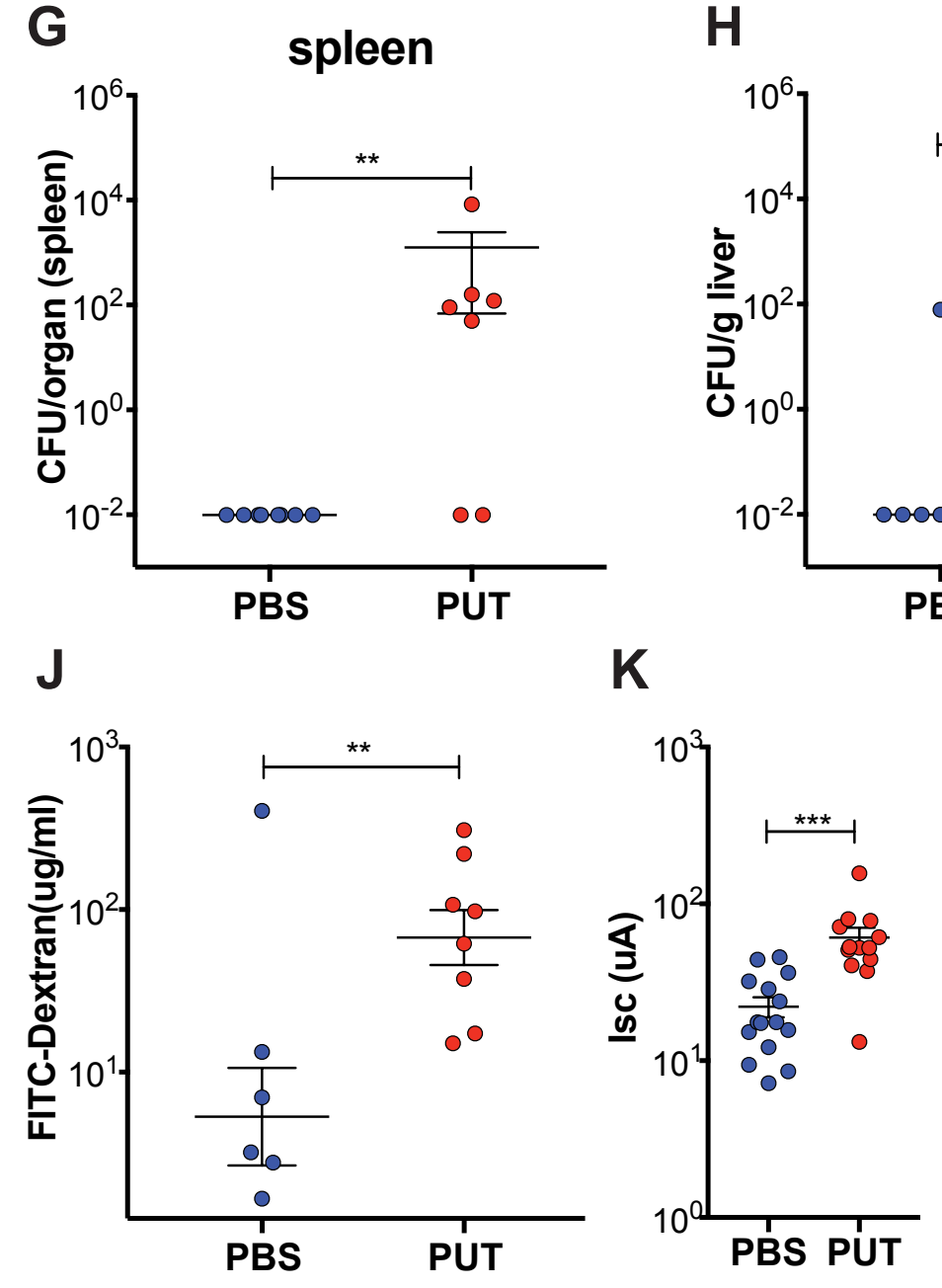

Supplemental figure 10

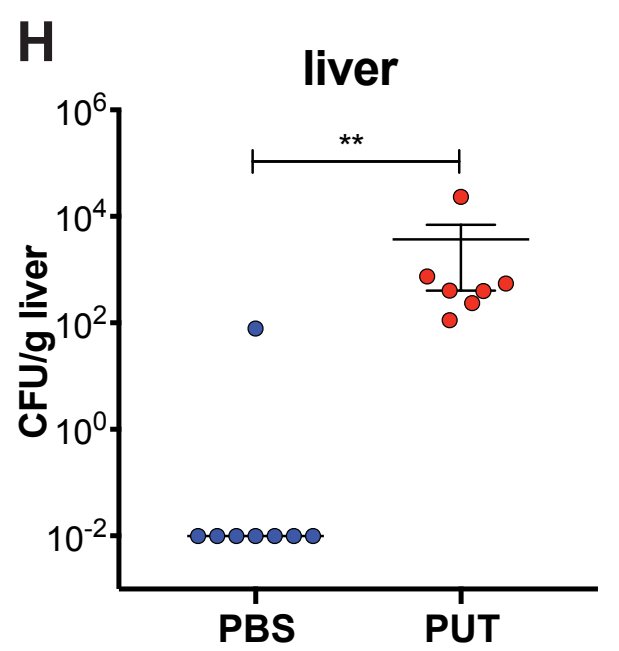

E

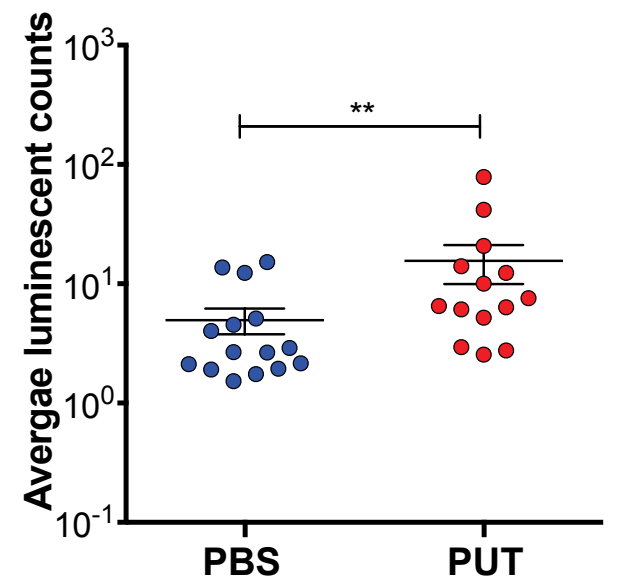

H
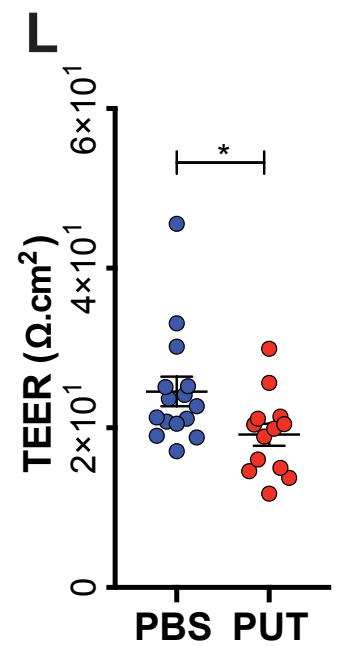

C

ભ

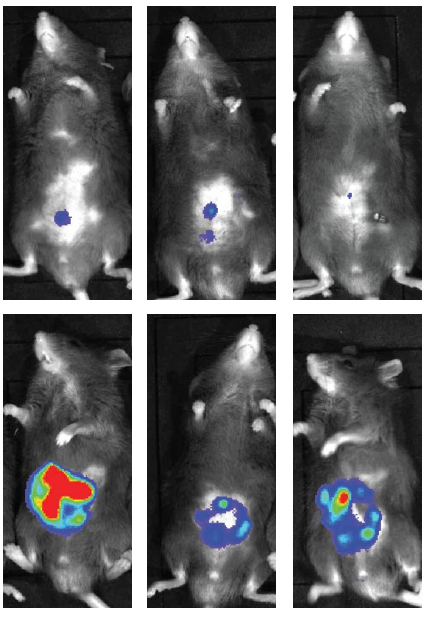

F

ભ
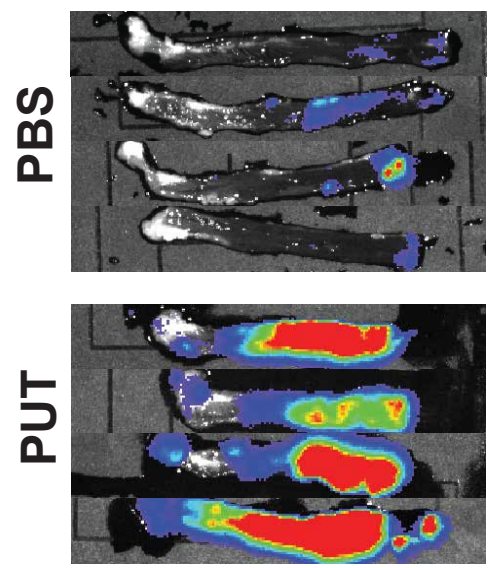

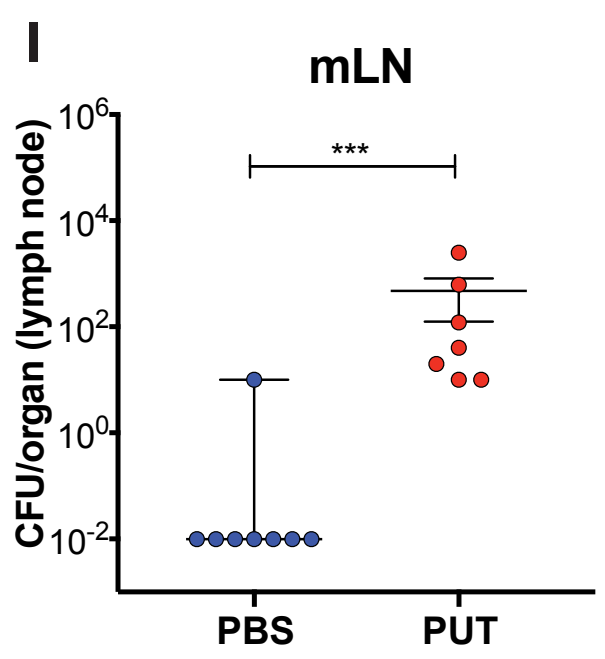


A
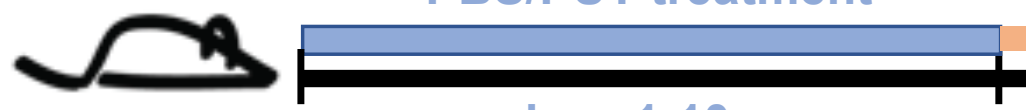

days 1-10

B
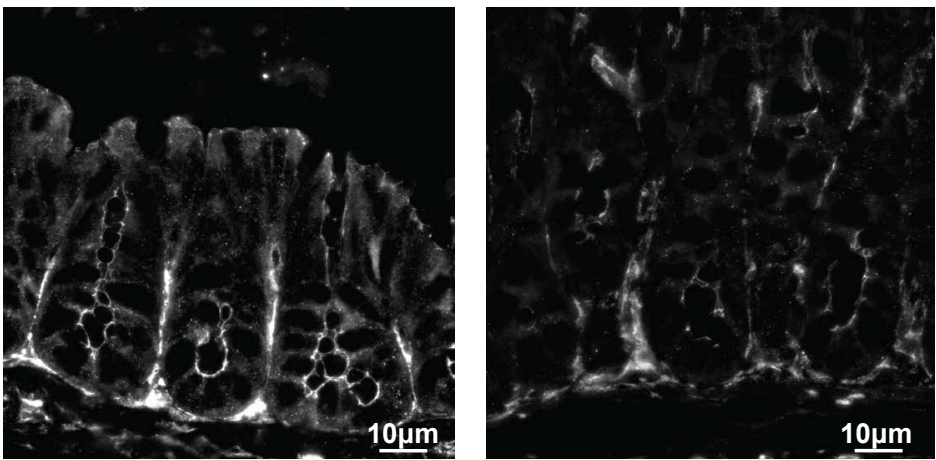

D

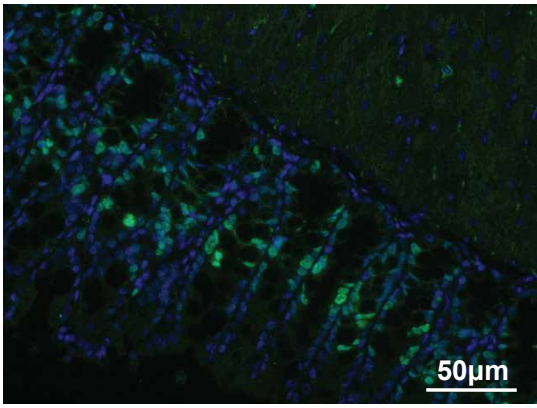

PBS

F

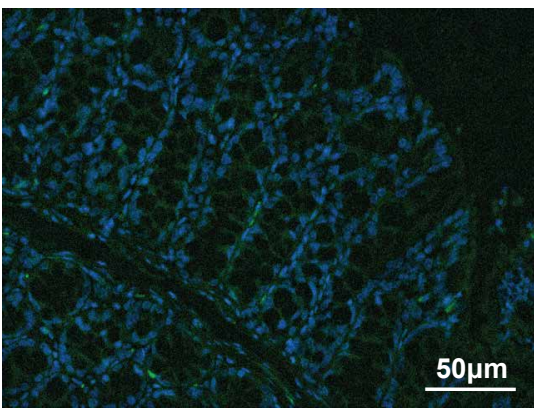

PBS

$H$
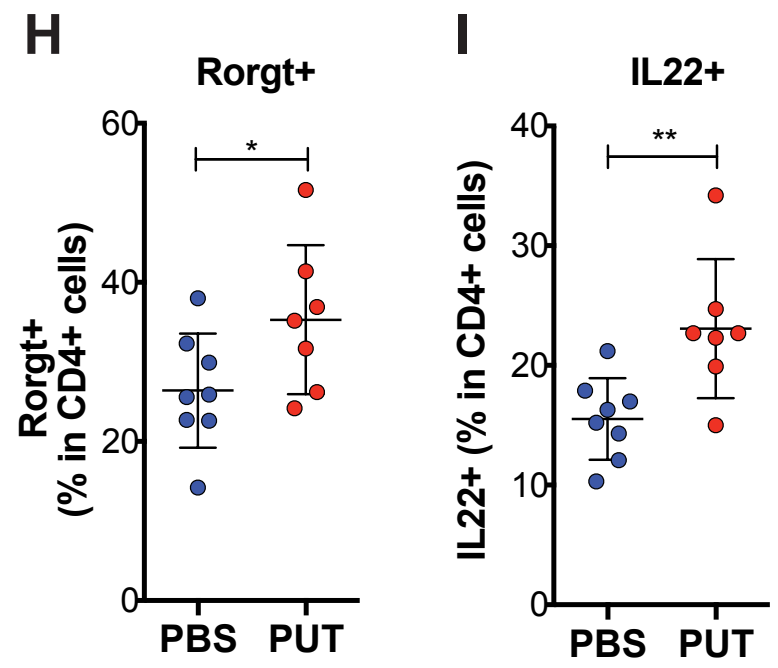

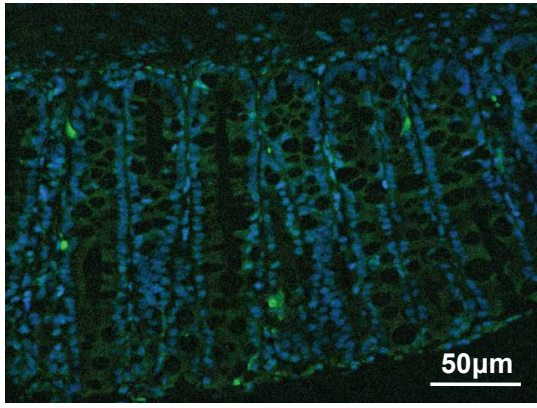

PUT
C

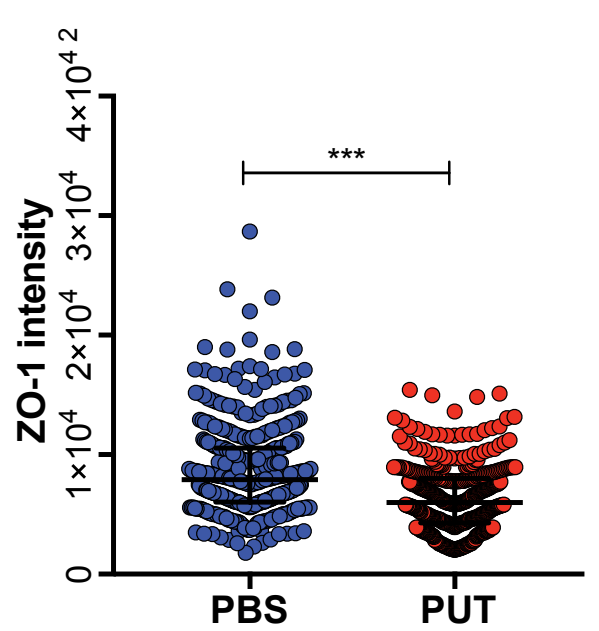

E

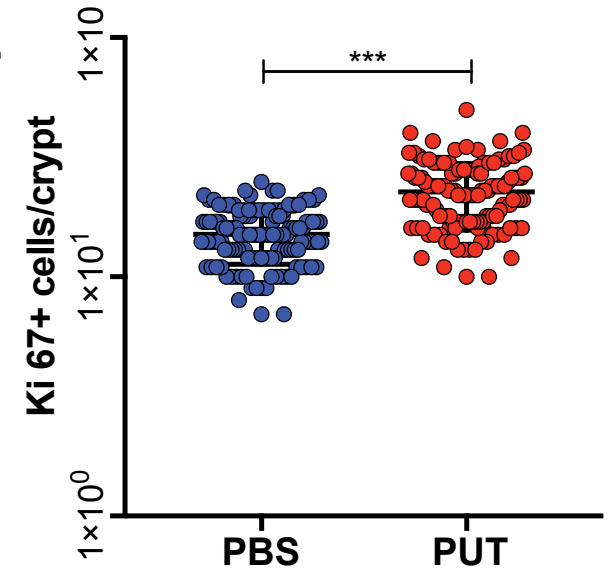

DAPI

Caspase 3

G

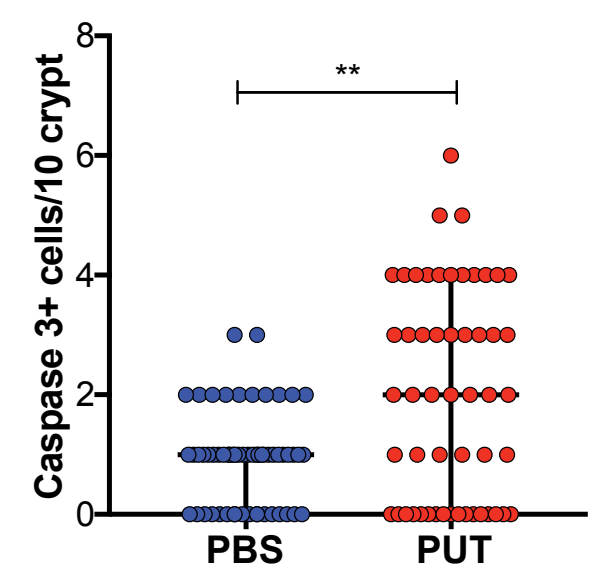

Supplemental figure 11
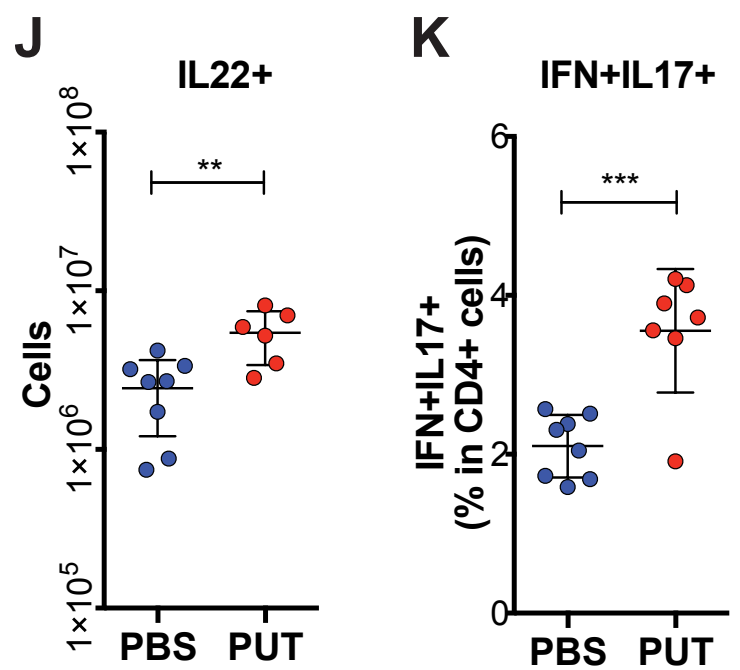

L

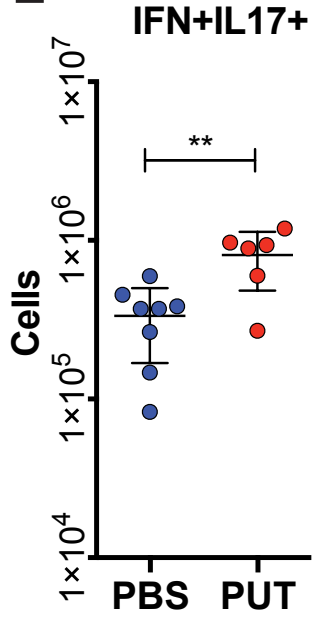


A

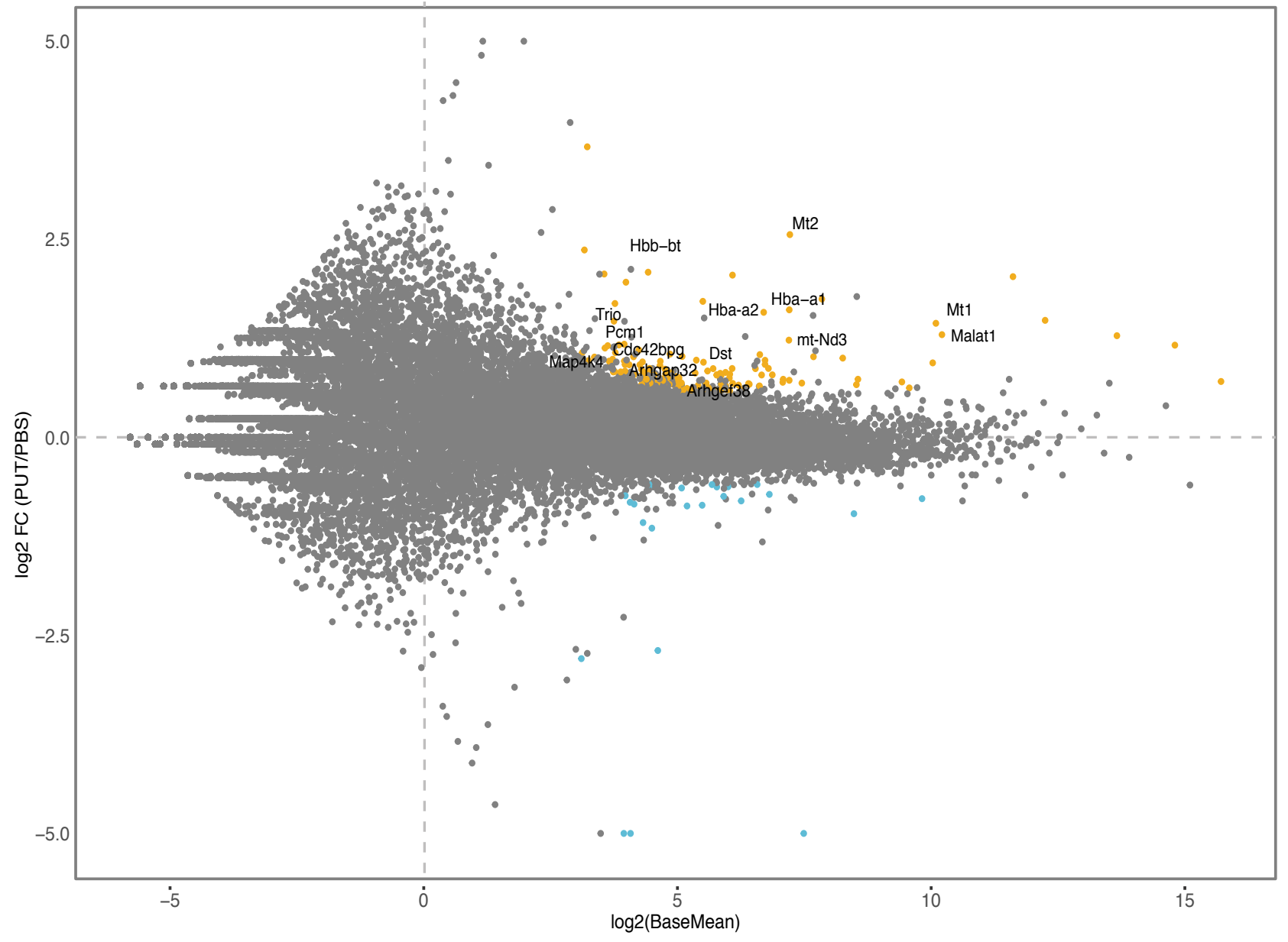

B

small GTPase binding actin binding guanyl-nucleotide exchange factor activity adherens junction anchoring junction $0.025 \quad 0.050 \quad 0.075$

GeneRatio

C

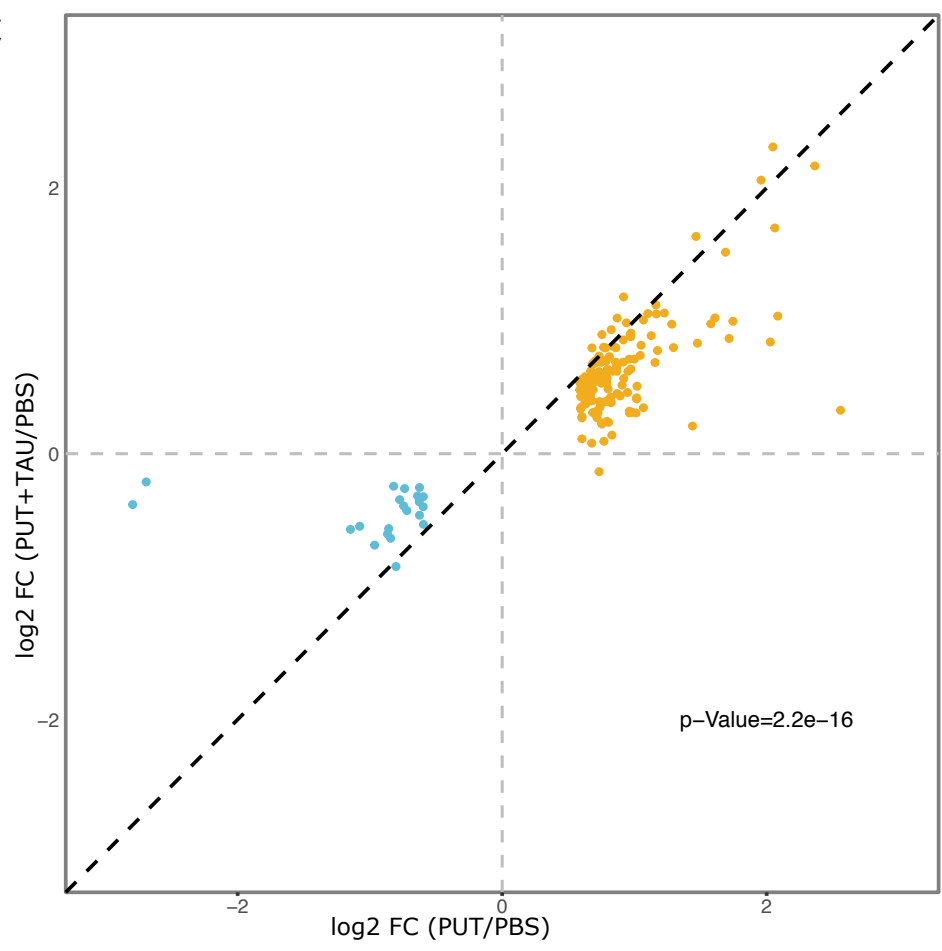

Supplemental figure 12 


\section{Supplemental Figure legends}

Supplemental figure 1. 3-dimensional organization of the apical cell-cell junction and basal cell-ECM adhesions in cultured CaCo- 2 cells. CaCo- 2 cells were cultured for 48 hours on collagen-coated glass coverslips, fixed-permeabilized and labeled for ZO-1 (A, B (left panel), green in the colored images), zyxin ( $A, C$ (left panel), light blue in colored images), actin ( $A, B, C$, red in colored images) and DNA ( $A$, dark blue). Z-stack was acquired to cover the entire volume of the epithelial monolayer and subjected to deconvolution. (A) Projection of all slices (left) and side view (right) of the 3D volume, (B) apical domain (left-to-right: ZO-1, actin, merged, enlarged section outlined in the merged image), (C) basal domain (left-to-right: zyxin, actin, merged, enlarged section outlined in the merged image). (D) Images subjected to automated segmentation for identification of TJ and FA. All images are derived from the same multi-color Z-stack

Supplemental figure 2. Effects of human secreted molecules on TJ and FA integrity. CaCo-2 cells were plated on collagen-coated glass-bottom 96 well plates, and treated with the library of human secreted molecules (secretome library) for 24 hours. Cells were then fixed and labeled with antibodies for the cingulin and paxilin to visualize TJs and FAs, respectively. (A) Representative examples of TypeI disrupted TJs and the corresponding elongation of FAs, induced by the indicated molecules. (B) Representative examples of TJs and FA morphology induced by TJ stabilizers. The effective concentrations are listed in Supplemental Methods. (C) Quantification of TJ tortuosity. (D) Quantification of FA axial ratios, induced by the indicated treatments.

\section{Supplemental figure 3. Newly identified bacterial metabolites capable of disrupting or stabilizing} TJs. CaCo-2 cells were plated on collagen-coated glass-bottom 96 well plates and treated with a set of 25 bacterial metabolites, either alone or in combination with $100 \mathrm{ng} / \mathrm{ml}$ of LPS (a TJ disruptor). After 24 hours, cells were fixed and labeled with antibodies for cingulin and paxilin to visualize TJs and FAs, respectively. (A) Non-treated control, (B-D) three metabolites inducing Type-I and Type-II TJ disruption and concomitantly enhancing FAs (outlined in red). (E) Cell treated with LPS only, (F-H) three metabolites capable of blocking LPS-induced changes in TJ and FA morphology (outlined in blue).

Supplemental figure 4. Selected examples of pharmacologically active compounds, capable of stabilizing or disrupting TJs. CaCo-2 cells were plated on collagen coated glass-bottom 96 well plates and treated with a compound library (SelleckChem), consisting of 2956 compounds, screened at $10 \mu \mathrm{M}$ concentration, in the presence of $100 \mathrm{ng} / \mathrm{ml}$ LPS and $10 \mathrm{mM}$ histamine (A) or alone (B). After 24 hours of treatment, the cells were fixed and labeled with antibodies for ZO-1 and zyxin to visualize TJs and FAs, respectively. (A) Examples of two drugs flavopiridol and daunomycine), capable of preventing the LPS/Histamine-induced changes in TJ and FA morphology. (B) examples of two drugs 
inducing Type-I (AT7867) or type-II (Vincristine) TJ disruption, and the corresponding enhancement of FAs.

Supplemental figure 5. Epithelial stabilizers can restore pre-existing TJ disruption. (A) CaCo-2 cells were treated as indicated for 48 hours, fixed and labeled with anti-ZO1 antibodies to visualize TJs. After initial 24 hours of treatment with disruptors (LPS, histamine, putrescin), the cells were subjected to another 24 hours with the same disruptors, or with fresh culture medium, in the presence or absence of a stabilizer (taurine or tryptamine). (B) Quantification of TJ disruption (tortuosity, for assessing Type-I phenotype) after LPS treatment, with all indicated combinations. (C) Quantification of TJ roughness for treatments causing Type-II phenotype with all restorative combinations.

\section{Supplemental figure 6. Epithelial disruptors and stabilizers modulate TJ morphology in T84 cells.} T84 cells were treated with either individual disruptors (A) or stabilizers (left column) or with their mixtures (B, C). Following treatment for 24 hours, the cells were fixed and labeled with anti-ZO1 antibodies.

Supplemental figure 7. Upregulation of acto-myosin contractility causes TJ disruption and can be prevented by epithelial stabilizers. (A) CaCo-2 cells were treated with various doses of Calyculin A, for three different time intervals as indicated, then, fixed and labeled for ZO-1 for TJ visualization. (B) CaCo- 2 cells were treated either with mixture of Calyculine A with epithelial stabilizers for 1 hour, or pre-incubated for 24 hours with individual stabilizers, and then subjected to combined treatment with Calyculin A/stabilizer mixture for 1 hour. Cells were then fixed and labeled with anti-ZO-1 antibodies.

Supplemental figure 8. Effect of putrescine on $\mathrm{TJ}$ integrity in an intestinal organ culture system and in mice with DSS-induced colitis before the onset of inflammation. (A) Pictures showing the ex vivo three-dimensional colon culture system used for the perfusion of different concentrations of putrescine through colon lumens. (B) Representative histology images of colon tissues cultured with different concentrations of putrescine solution for 2 hours. (C) ZO-1 staining of colon tissues cultured with control medium or putrescine $(1: 300,33.2 \mathrm{mM})$. (D-G) Wildtype mice with oral gavage of putrescine or PBS were administered with $2 \%$ DSS in the drinking water for 5 days ( $n=8$ mice per group). (D) Schematic illustration demonstrating setting of the experiment. (E) Weight changes after DSS administration until day 5. (F-G) Pattern recognition receptor stimulation by spleen (F) and liver (G) extracts from mice on day 5 after DSS administration. Ctrl: control medium; PUT: putrescine. 
Supplemental figure 9. Disruptive effect of putrescine in mice with DSS-induced colitis. Acute DSS colitis (2\% DSS in drinking water for 7 days) was induced in WT mice with oral gavage of putrescine or PBS ( $n=8-10$ mice in each group). (A) Schematic illustration demonstrating setting of the experiment. (B) comparison of daily DSS consumption between groups. Weight loss (C), survival (D), representative colonoscopy images on day7 (E), colonoscopy severity score on day 7 (F), measurement of colon lengths on day $12(\mathrm{G}-\mathrm{H})$, representative histology images on day $12(\mathrm{I})$, the pathology scoring on day 12 after DSS treatment(J). PUT: putrescine.

Supplemental figure 10. Disruptive effect of putrescine in mice with $C$. rodentium infection. Wildtype mice with oral gavage of PBS or putrescine were infected with $C$. rodentium ( $n=8-15$ mice per group). (A-C) CFUs recovered from stool (A), abdominal bioluminescence quantification (B) and imaging $(C)$ during the post-infection course. Comparison of the area under the curve (AUC) between PBS and putrescine group were shown in the insets of (A) and (B). (D-F) CFUs recovered from colonic tissue (D), ex vivo colonic bioluminescence quantification (E) and imaging (F) on day 8 post infection. (G-I) CFUs recovered from spleens (G), livers (H) and lymph nodes (I) on day 8 post infection. (J-L) FITC-dextran levels recovered from the serum 3 hours after oral gavage $(80 \mathrm{mg} / \mathrm{ml}$ FITC-dextran) on day $5(\mathrm{~J})$, Ussing chamber recording of short circuit current (ISC) (K) and trans-epithelial electrical resistance across colon epithelial layer (L) on day 8 post infection. PUT: putrescine.

Supplemental figure 11. Disruptive effect of putrescine in mice with $C$. rodentium infection. Wildtype mice with oral gavage of putrescine or PBS were infected with $C$. rodentium ( $n=8-15$ mice each group). (A) Schematic illustration demonstrating setting of the experiment. (B-C) ZO-1 staining (B) and quantification (C) of colonic sections on day 8 post infection. (D-G) Ki-67 staining (D) and quantification (E), cleaved caspase-3 staining (F) and quantification (G) of colonic sections at day 8 post infection. ( $\mathrm{H}-\mathrm{L})$ Flow cytometry enumeration of Th17 (RORgt+) subsets $(\mathrm{H})$, secretion of inflammatory cytokine IL-22 (I-J), IL-17 and Interferon-gamma (K-L) of hematopoietic cells harvested from the lamina propria of mice on day 8 post infection. PUT: putrescine.

Supplemental figure 12. Transcriptional profiling of sorted EpCAM+ colonic epithelial cells in mice treated with PBS, putrescine, putrescine plus taurine ( $n=10$ mice per group). (A) Differentially expressed genes in the epithelial cells of PBS- and putrescine-treated mice (genes upregulated in the putrescine group with adjusted $p$-value $<0.05$ are in orange). (B) Pathway enrichment analysis of differentially expressed genes. (C) Fold-change (FC) versus FC plot comparing the transcriptional signatures induced by putrescine treatment ( $x$-axis) and putrescine plus taurine treatment ( $y$-axis) (genes upregulated by putrescine are in orange). 


\section{References.}

1. Revach, O. Y., Winograd-Katz, S. E., Samuels, Y. \& Geiger, B. The involvement of mutant Rac1 in the formation of invadopodia in cultured melanoma cells. Experimental Cell Research 343, 82-88 (2016).

2. Legland, D., Arganda-Carreras, I. \& Andrey, P. MorphoLibJ: Integrated library and plugins for mathematical morphology with ImageJ. Bioinformatics 32, 3532-3534 (2016).

3. Schindelin, J. et al. Fiji: An open-source platform for biological-image analysis. Nature Methods 9, 676-682 (2012).

4. Shee, K. et al. Therapeutically targeting tumor microenvironment-mediated drug resistance in estrogen receptor-positive breast cancer. J. Exp. Med. 215, 895-910 (2018).

5. Levy, M. et al. Microbiota-Modulated Metabolites Shape the Intestinal Microenvironment by Regulating NLRP6 Inflammasome Signaling. Cell 163, 1428-1443 (2015).

6. Butler, J. P., Tolić-Nørrelykke, I. M., Fabry, B. \& Fredberg, J. J. Traction fields, moments, and strain energy that cells exert on their surroundings. Am. J. Physiol. Physiol. 282, C595-C605 (2013).

7. Yissachar, N. et al. An Intestinal Organ Culture System Uncovers a Role for the Nervous System in Microbe-Immune Crosstalk. Cell 168, 1135-1148.e12 (2017).

8. Becker, C., Fantini, M. C. \& Neurath, M. F. High resolution colonoscopy in live mice. Nat. Protoc. 1, 2900-2904 (2007).

9. Wirtz, S. et al. Chemically induced mouse models of acute and chronic intestinal inflammation. Nat. Protoc. 12, 1295-1309 (2017).

10. Wilson, G. et al. Transport and permeability properties of human Caco-2 cells: An in vitro model of the intestinal epithelial cell barrier. J. Control. Release 11, 25-40 (1990).

11. Odenwald, M. A. \& Turner, J. R. The intestinal epithelial barrier: a therapeutic target? Nat. Rev. Gastroenterol. Hepatol. 14, 9-21 (2017).

12. Thaiss, C. A. et al. Hyperglycemia drives intestinal barrier dysfunction and risk for enteric infection. Science (80-. ). 359, 1376-1383 (2018).

13. Dandapani, S., Rosse, G., Southall, N., Salvino, J. M. \& Thomas, C. J. Selecting, Acquiring, and Using Small Molecule Libraries for High-Throughput Screening. in Current Protocols in Chemical Biology 4, 177-191 (John Wiley \& Sons, Inc., 2012).

14. Devriese, S. et al. T84 monolayers are superior to Caco-2 as a model system of colonocytes. Histochem. Cell Biol. 148, 85-93 (2017).

15. Cunningham, K. E. \& Turner, J. R. Myosin light chain kinase: pulling the strings of epithelial tight junction function. Ann. N. Y. Acad. Sci. 1258, 34-42 (2012).

16. Butler, J. P., Tolić-Nørrelykke, I. M., Fabry, B. \& Fredberg, J. J. Traction fields, moments, and strain energy that cells exert on their surroundings. Am. J. Physiol. Physiol. 282, C595-C605 (2002).

17. Suzuki, A. \& Itoh, T. Effects of calyculin A on tension and myosin phosphorylation in skinned smooth muscle of the rabbit mesenteric artery. Br. J. Pharmacol. 109, 703-712 (1993).

18. Wang, F. et al. Interferon- $\gamma$ and tumor necrosis factor- $\alpha$ synergize to induce intestinal epithelial barrier dysfunction by up-regulating myosin light chain kinase expression. Am. J. Pathol. 166, 409419 (2005). 